\begin{tabular}{|c|c|}
\hline Title & Bay esian Modeling of Enteric V irus Density in W astewater U sing Left-Censored Data \\
\hline Author(s) & Kato, Tsuy oshi; Miura, Takay uki; Okabe, Satoshi; Sano, Daisuke \\
\hline Citation & $\begin{array}{l}\text { Food and Environmental Virology, 5(4), 185-193 } \\
\text { https:/doi.org/10.1007/s12560-013-9125-1 }\end{array}$ \\
\hline Issue Date & $2013-12$ \\
\hline DOC URL & http:/hdl.handle.net/2115/57531 \\
\hline Rights & The final publication is avail able at link.springer.com \\
\hline Type & article (author version) \\
\hline Additional Information & There are other files related to this item in HUSCAP. Check the above URL. \\
\hline File Information & Kato2013SupportingI nformationv2.pdf (Supporting Information) \\
\hline
\end{tabular}

Instructions for use 


\title{
Supporting Information: \\ Bayesian modeling of enteric virus density in wastewater using left-censored data
}

\author{
Tsuyoshi Kato ${ }^{1,2}$, Takayuki Miura ${ }^{3}$, Satoshi Okabe $^{3}$, and Daisuke Sano ${ }^{3, *}$
}

Each author's affiliation and mailing address:

1 Department of Computer Science, Graduate School of Engineering, Gunma University, Tenjinmachi 1-5-1, Kiryu, Gunma 376-8515, Japan

2 Center for Informational Biology, Ochanomizu University, Otsuka 2-1-1, Tokyo 112-8610, Japan

3 Division of Environmental Engineering, Faculty of Engineering, Hokkaido University, North 13, West 8, Kita-ku, Sapporo, Hokkaido 060-8628, Japan

* Corresponding Author. Division of Environmental Engineering, Faculty of Engineering, Hokkaido University, North 13, West 8, Kita-ku, Sapporo, Hokakaido 060-8628, Japan. Telephone/Fax: +81-11-706-7597. E-mail: dsano@eng.hokudai.ac.jp

\section{Figures 1S-39S}

We simulated three situations: The numbers of samples gathered from treated wastewater, $n$, were 12, 24 , and 48 . For the dataset with $n=12$ samples, we considered twelve cases where the numbers of detect-data, say $n_{v}$, were $1,2, \ldots, 12$. For $n=24$, we added a case of $n_{v}=24$. For $n=48$, we further added a case of $n_{v}=48$. Totally, we considered $12+13+14=39$ cases. Each of Figures 1S-39S shows the Bayesian inferences for one of 39 cases.

For each case of $\left(n, n_{v}\right)$, a hundred datasets of treated wastewater were generated for our simulation. We here denoted the $k$-th dataset by $\mathcal{X}_{k \text {,post }}$. The common logarithm of concentration data in each dataset were generated according to a normal distribution $\mathcal{N}(1,1)$, but the detection limit $\theta_{v}$ was adjusted so that the number of non-detect data was exactly $n_{0}$. In the panels (a) of each figure, the probabilistic density function of the normal distribution $\mathcal{N}(1,1)$ is plotted in red color with a vertical line segmented indicating the location of the detection limit for the first five datasets, $\mathcal{X}_{1, \text { post }}, \mathcal{X}_{2 \text {,post }}, \ldots, \mathcal{X}_{5, \text { post }}$. In the panels $(\mathrm{a})$, red circles and blue circles represent the common logarithms of detect data and non-detect data, respectively.

Furthermore, for each of the treated wastewater datasets, an untreated wastewater dataset, denoted by $\mathcal{X}_{k \text {,pre, was }}$ generated to be coupled with the corresponding $\mathcal{X}_{k \text {,post }}$. The common logarithms of concentration data in $\mathcal{X}_{k \text {,pre }}$ were generated according to $\mathcal{N}(4,1)$, where the number of samples is $n$. In $\mathcal{X}_{k \text {,pre }}$, the generated data less than $\theta_{v}$ were treated as non-detect data, where $\theta_{v}$ was equal to the value used to generate the corresponding $\mathcal{X}_{k \text {,post. }}$. In the panels (d) of each figure, the density function of $\mathcal{N}(4,1)$ is plotted in red color for the first five datasets, $\mathcal{X}_{1 \text {,pre }}, \mathcal{X}_{2, \text { pre }}, \ldots$, $\mathcal{X}_{5, \text { pre }}$. The common logarithms of detect data and non-detect data, respectively, were also depicted with red circles and blue circles.

Panels $(\mathrm{g}-1), \ldots,(\mathrm{g}-5)$ use contours to show the posterior distributions of model parameters for treated wastewater datasets, say $p\left(\mu, \beta \mid \mathcal{X}_{k, \text { post }}\right)$. Panels $(\mathrm{h}-1), \ldots,(\mathrm{h}-5)$ are for untreated wastewater datasets.

Marginal posteriors, $p\left(\mu \mid \mathcal{X}_{k \text {,post }}\right)$ and $p\left(\beta \mid \mathcal{X}_{k \text {,post }}\right)$, for the first five treated wastewater datasets are plotted in (b) and (c), respectively. Panels (e) and (f) are of the untreated wastewater datasets.

Black curves in (a) are the posterior predictive distribution $p\left(c_{\text {post }} \mid \mathcal{X}_{k, \text { post }}\right)$, inferred from a treated wastewater dataset. Ideally, the posterior predictive distributions would approach to the true distribution $\mathcal{N}(1,1)$, depicted with red curves. Black curves in (d) are for untreated wastewater datasets.

Panels (i-1),..,(i-5) depict the log ratio posteriors that are the posterior distribution of the log ratio between concentration data in untreated wastewater and those in treated wastewater. For comparison, the true distribution $\mathcal{N}(3,2)$ is also drawn in each plot. 


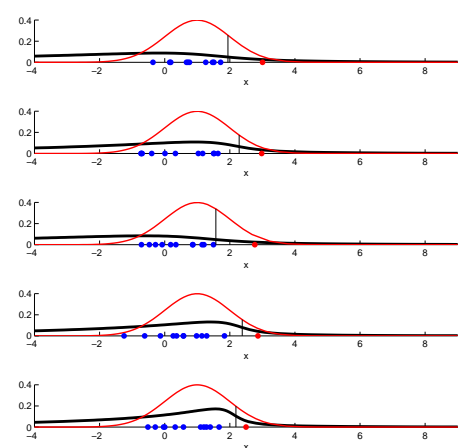

(a) $p\left(c_{\text {post }} \mid \mathcal{X}_{k, \text { post }}\right)$

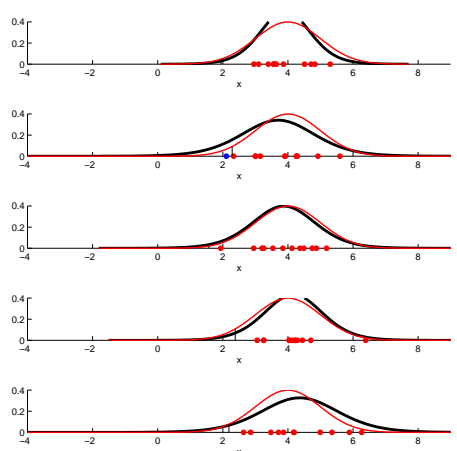

(d) $p\left(c_{\text {pre }} \mid \mathcal{X}_{k, \text { pre }}\right)$

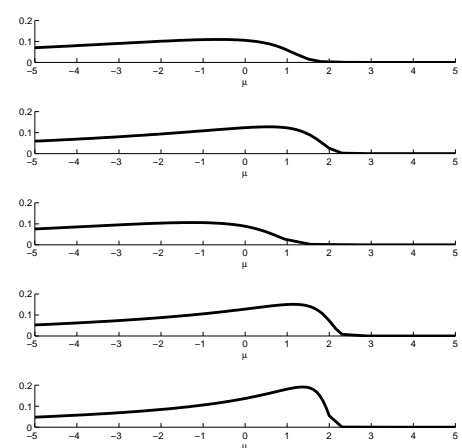

(b) $p\left(\mu_{1} \mid \mathcal{X}_{k, \text { post }}\right)$

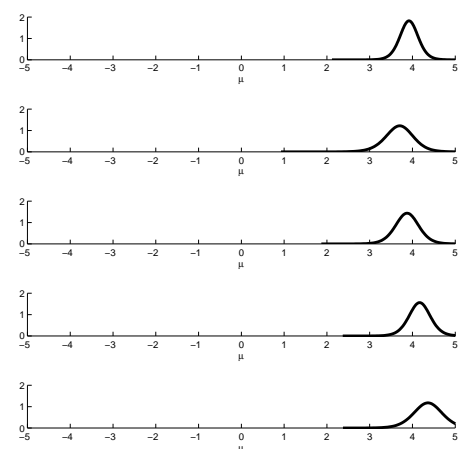

(e) $p\left(\mu_{1} \mid \mathcal{X}_{k, \text { pre }}\right)$

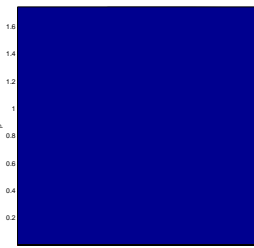

(g-3) $p\left(\mu, \beta \mid \mathcal{X}_{3, \text { post }}\right)$

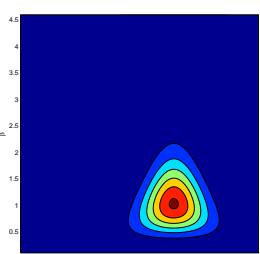

(h-3) $p\left(\mu, \beta \mid \mathcal{X}_{3, \text { pre }}\right)$

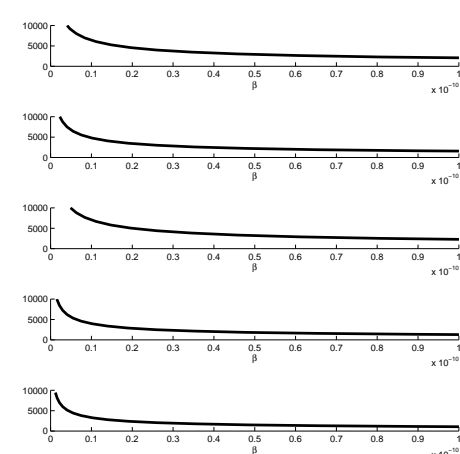

(c) $p\left(\beta_{1} \mid \mathcal{X}_{k, \text { post }}\right)$

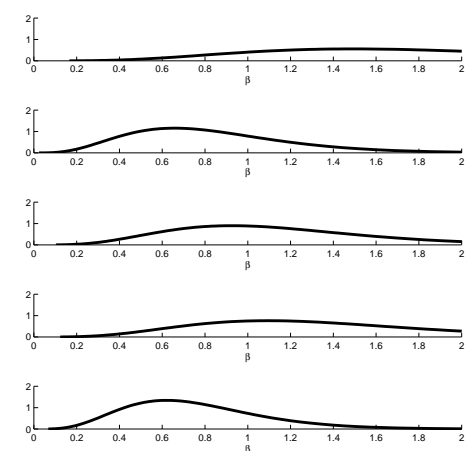

(f) $p\left(\beta_{1} \mid \mathcal{X}_{k, \text { pre }}\right)$
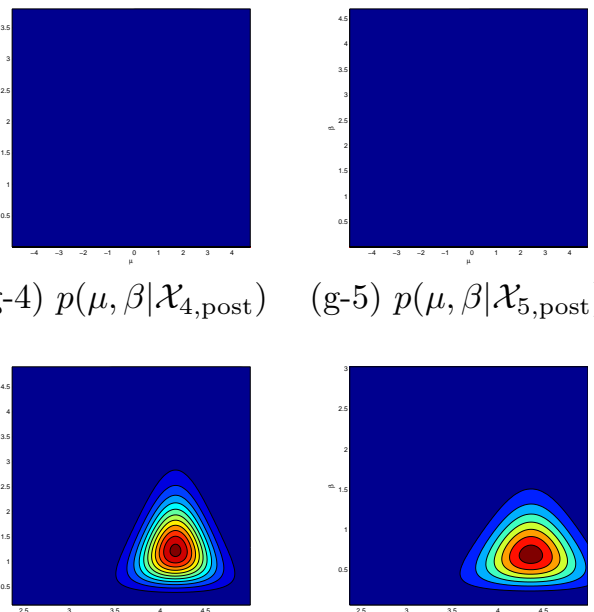

(g-4) $p\left(\mu, \beta \mid \mathcal{X}_{4, \text { post }}\right)$

(g-5) $p\left(\mu, \beta \mid \mathcal{X}_{5, \text { post }}\right)$

(h-4) $p\left(\mu, \beta \mid \mathcal{X}_{4, \text { pre }}\right)$

(h-5) $p\left(\mu, \beta \mid \mathcal{X}_{5, \text { pre }}\right)$ (h-1) $p\left(\mu, \beta \mid \mathcal{X}_{1, \text { pre }}\right)$

(g-1) $p\left(\mu, \beta \mid \mathcal{X}_{1, \text { post }}\right)$
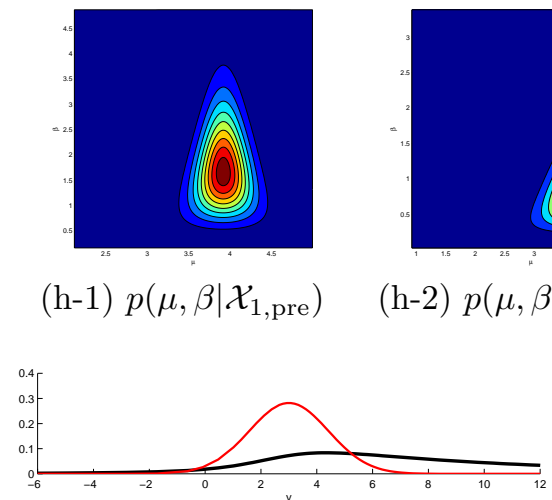

(i-1) $p\left(y \mid \mathcal{X}_{1, \text { pre }}, \mathcal{X}_{1, \text { post }}\right)$

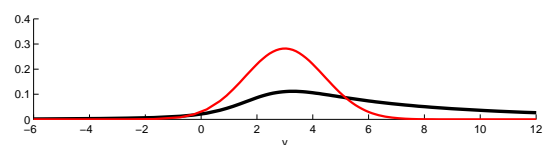

(i-4) $p\left(y \mid \mathcal{X}_{4, \text { pre }}, \mathcal{X}_{4, \text { post }}\right)$

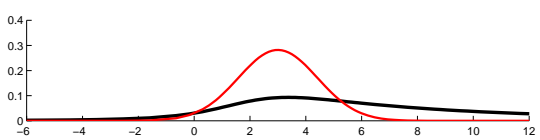

(i-2) $p\left(y \mid \mathcal{X}_{2, \text { pre }}, \mathcal{X}_{2, \text { post }}\right)$

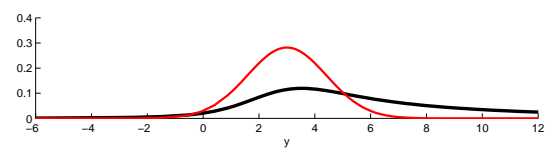

(i-5) $p\left(y \mid \mathcal{X}_{5, \text { pre }}, \mathcal{X}_{5, \text { post }}\right)$

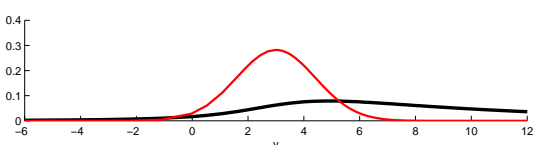

(i-3) $p\left(y \mid \mathcal{X}_{3, \text { pre }}, \mathcal{X}_{3, \text { post }}\right)$

Figure 1S: Bayesian inferences on five datasets, each of which contains $n_{v}=1$ detect data out of $n=12$ samples. 


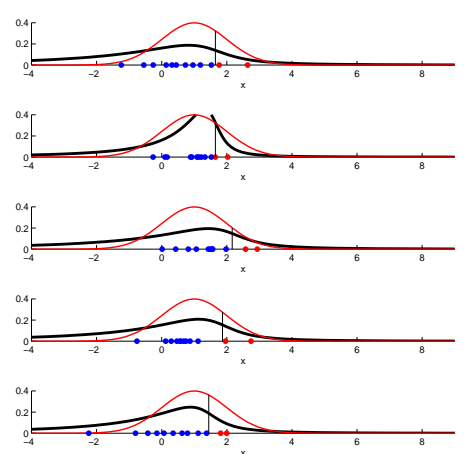

(a) $p\left(c_{\text {post }} \mid \mathcal{X}_{k \text {,post }}\right)$

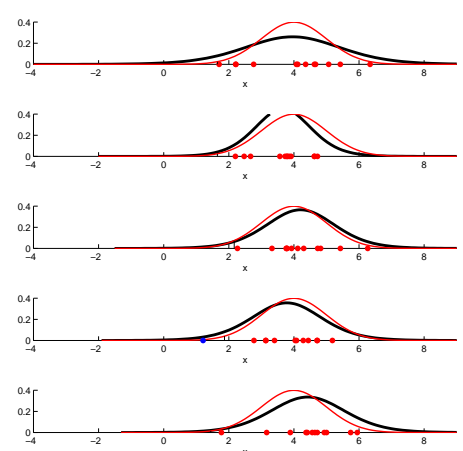

(d) $p\left(c_{\text {pre }} \mid \mathcal{X}_{k, \text { pre }}\right)$

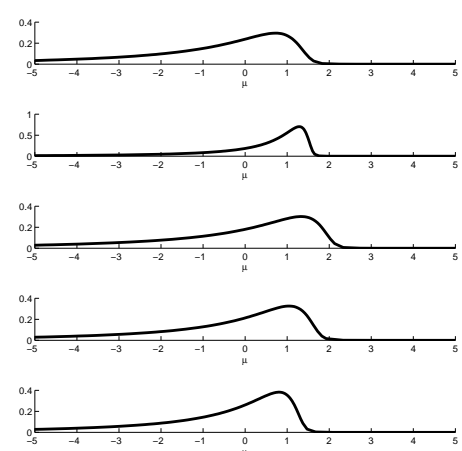

(b) $p\left(\mu_{1} \mid \mathcal{X}_{k, \text { post }}\right)$

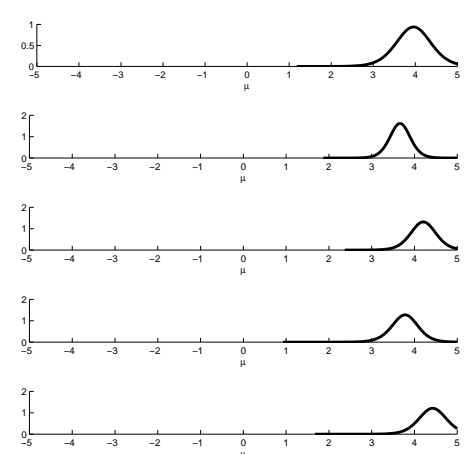

(e) $p\left(\mu_{1} \mid \mathcal{X}_{k, \text { pre }}\right)$

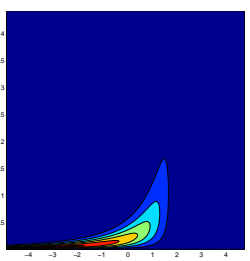

(g-3) $p\left(\mu, \beta \mid \mathcal{X}_{3, \text { post }}\right)$

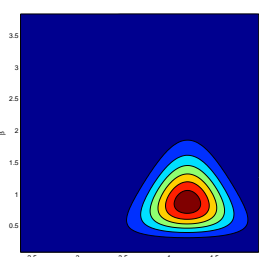

(h-3) $p\left(\mu, \beta \mid \mathcal{X}_{3, \text { pre }}\right)$

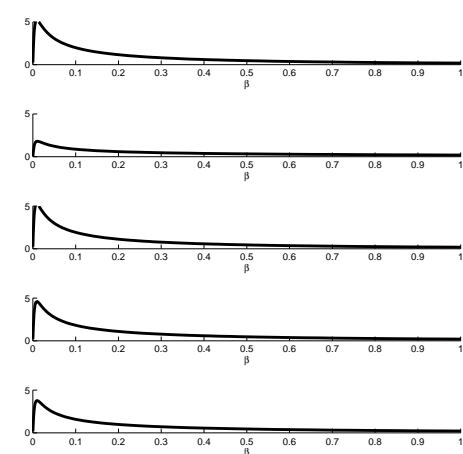

(c) $p\left(\beta_{1} \mid \mathcal{X}_{k, \text { post }}\right)$

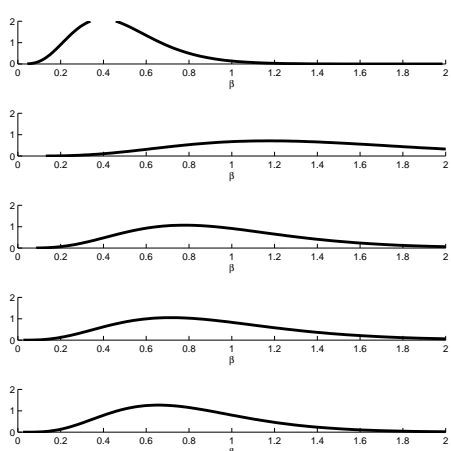

(f) $p\left(\beta_{1} \mid \mathcal{X}_{k, \text { pre }}\right)$

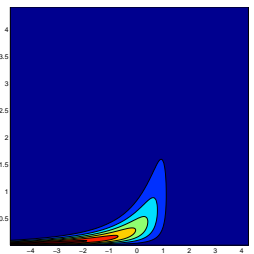

(g-1) $p\left(\mu, \beta \mid \mathcal{X}_{1, \text { post }}\right)$

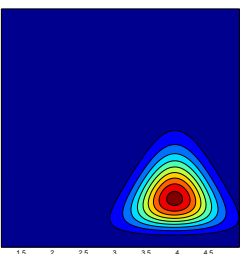

(h-1) $p\left(\mu, \beta \mid \mathcal{X}_{1, \text { pre }}\right)$

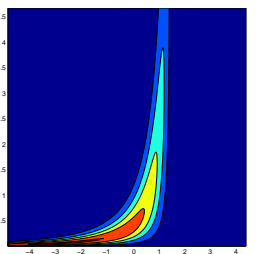

$(\mathrm{g}-2) p\left(\mu, \beta \mid \mathcal{X}_{2, \text { post }}\right)$

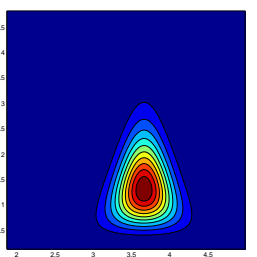

(h-2) $p\left(\mu, \beta \mid \mathcal{X}_{2, \text { pre }}\right)$

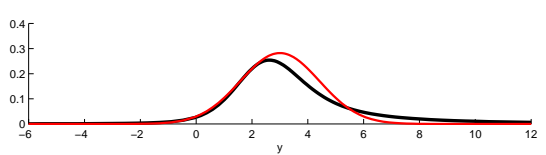

(i-2) $p\left(y \mid \mathcal{X}_{2, \text { pre }}, \mathcal{X}_{2, \text { post }}\right)$

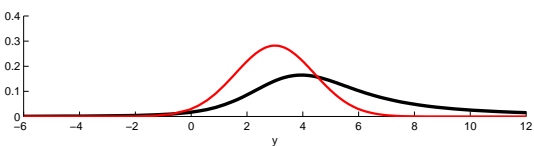

(i-5) $p\left(y \mid \mathcal{X}_{5, \text { pre }}, \mathcal{X}_{5, \text { post }}\right)$

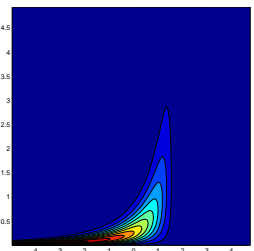

(g-4) $p\left(\mu, \beta \mid \mathcal{X}_{4, \text { post }}\right)$

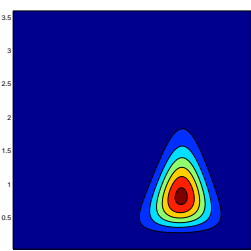

(h-4) $p\left(\mu, \beta \mid \mathcal{X}_{4, \text { pre }}\right)$

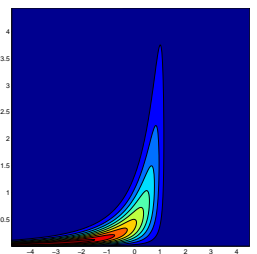

(g-5) $p\left(\mu, \beta \mid \mathcal{X}_{5, \text { post }}\right)$

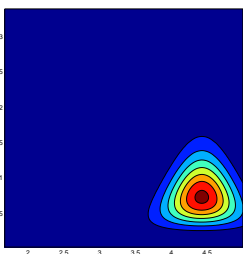

(h-5) $p\left(\mu, \beta \mid \mathcal{X}_{5, \text { pre }}\right)$

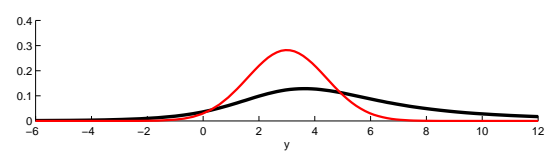

(i-1) $p\left(y \mid \mathcal{X}_{1, \text { pre }}, \mathcal{X}_{1, \text { post }}\right)$

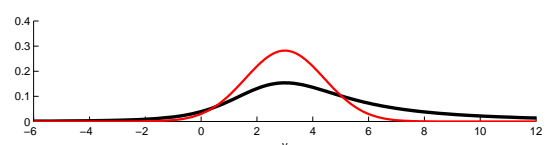

(i-4) $p\left(y \mid \mathcal{X}_{4, \text { pre }}, \mathcal{X}_{4, \text { post }}\right)$

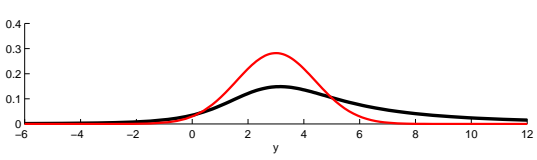

(i-3) $p\left(y \mid \mathcal{X}_{3, \text { pre }}, \mathcal{X}_{3, \text { post }}\right)$

Figure 2S: Bayesian inferences on five datasets, each of which contains $n_{v}=2$ detect data out of $n=12$ samples. 


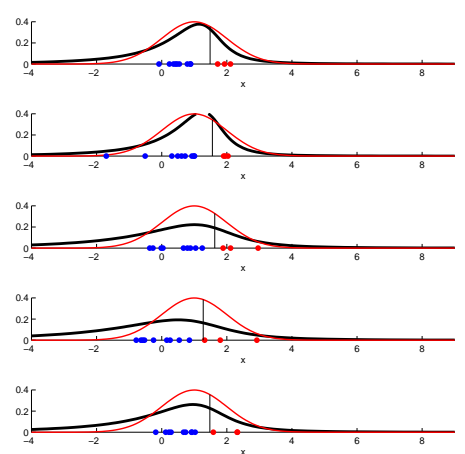

(a) $p\left(c_{\text {post }} \mid \mathcal{X}_{k, \text { post }}\right)$

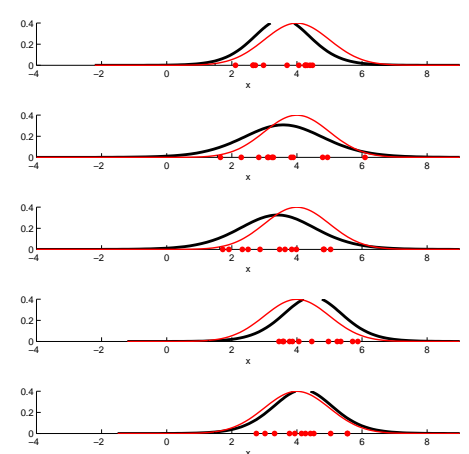

(d) $p\left(c_{\text {pre }} \mid \mathcal{X}_{k, \text { pre }}\right)$

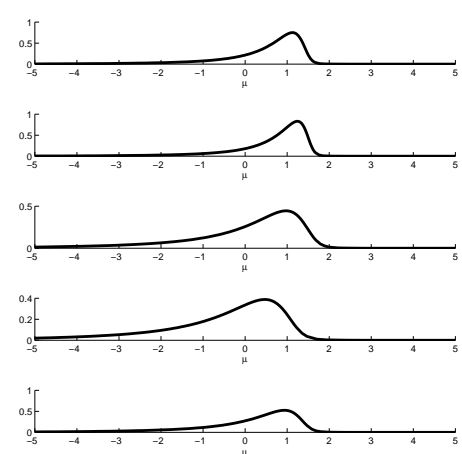

(b) $p\left(\mu_{1} \mid \mathcal{X}_{k, \text { post }}\right)$

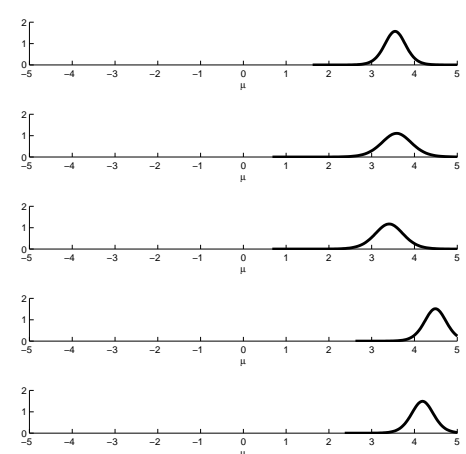

(e) $p\left(\mu_{1} \mid \mathcal{X}_{k, \text { pre }}\right)$

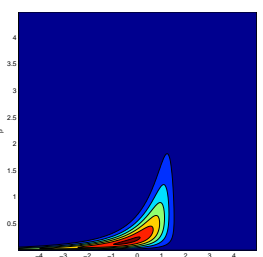

(g-3) $p\left(\mu, \beta \mid \mathcal{X}_{3, \text { post }}\right)$

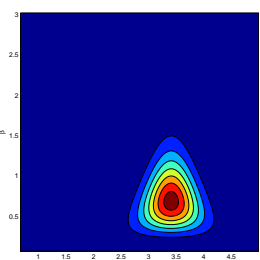

(h-3) $p\left(\mu, \beta \mid \mathcal{X}_{3, \text { pre }}\right)$

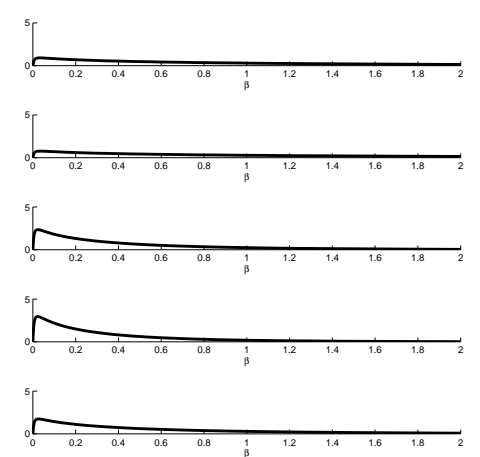

(c) $p\left(\beta_{1} \mid \mathcal{X}_{k, \text { post }}\right)$

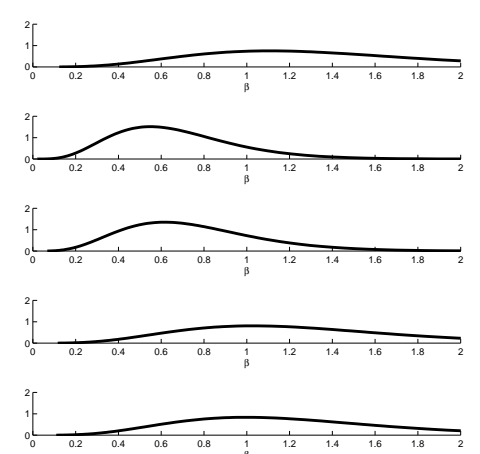

(f) $p\left(\beta_{1} \mid \mathcal{X}_{k, \text { pre }}\right)$

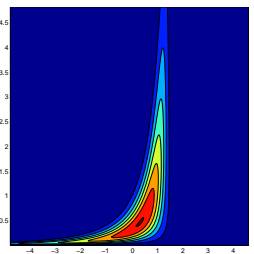

(g-1) $p\left(\mu, \beta \mid \mathcal{X}_{1, \text { post }}\right)$

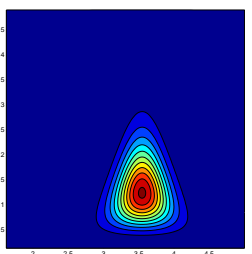

(h-1) $p\left(\mu, \beta \mid \mathcal{X}_{1, \text { pre }}\right)$

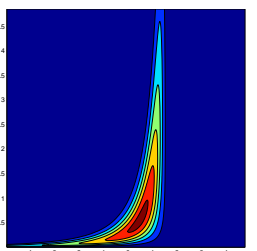

(g-2) $p\left(\mu, \beta \mid \mathcal{X}_{2, \text { post }}\right)$

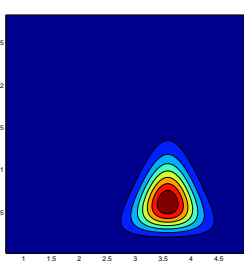

(h-2) $p\left(\mu, \beta \mid \mathcal{X}_{2, \text { pre }}\right)$

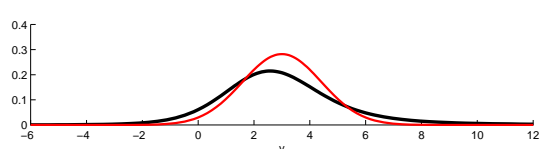

(i-2) $p\left(y \mid \mathcal{X}_{2, \text { pre }}, \mathcal{X}_{2, \text { post }}\right)$

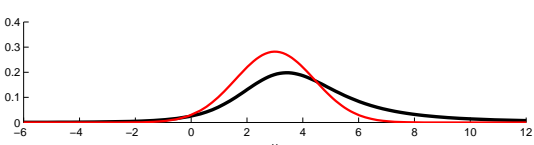

(i-5) $p\left(y \mid \mathcal{X}_{5, \text { pre }}, \mathcal{X}_{5, \text { post }}\right)$
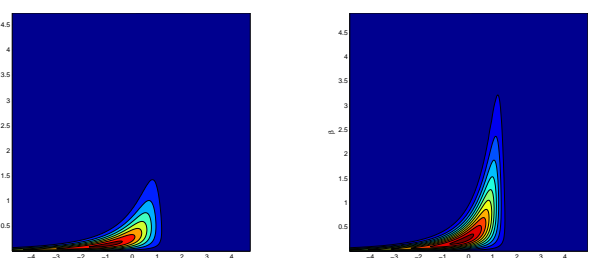

(g-4) $p\left(\mu, \beta \mid \mathcal{X}_{4, \text { post }}\right)$

(g-5) $p\left(\mu, \beta \mid \mathcal{X}_{5, \text { post }}\right)$
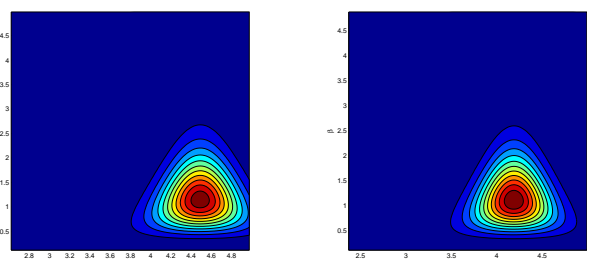

(h-4) $p\left(\mu, \beta \mid \mathcal{X}_{4, \text { pre }}\right) \quad(\mathrm{h}-5) p\left(\mu, \beta \mid \mathcal{X}_{5, \text { pre }}\right)$

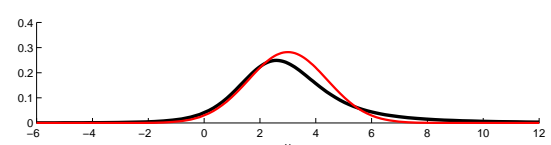

(i-1) $p\left(y \mid \mathcal{X}_{1, \text { pre }}, \mathcal{X}_{1, \text { post }}\right)$

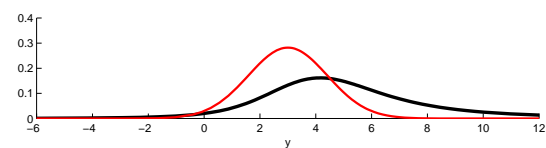

(i-4) $p\left(y \mid \mathcal{X}_{4, \text { pre }}, \mathcal{X}_{4, \text { post }}\right)$

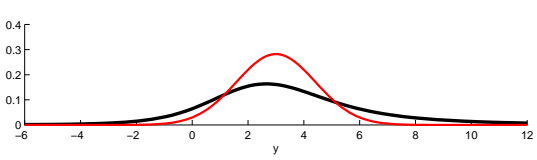

$(\mathrm{i}-3) p\left(y \mid \mathcal{X}_{3, \text { pre }}, \mathcal{X}_{3, \text { post }}\right)$

Figure 3S: Bayesian inferences on five datasets, each of which contains $n_{v}=3$ detect data out of $n=12$ samples. 


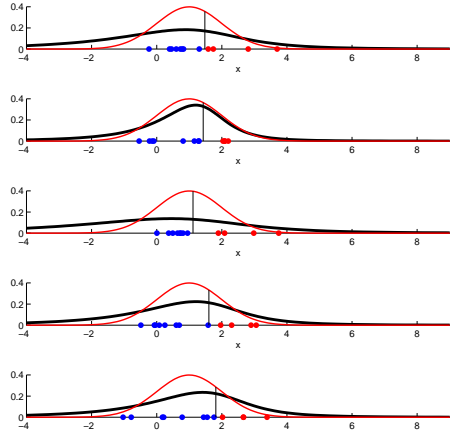

(a) $p\left(c_{\text {post }} \mid \mathcal{X}_{k \text {,post }}\right)$

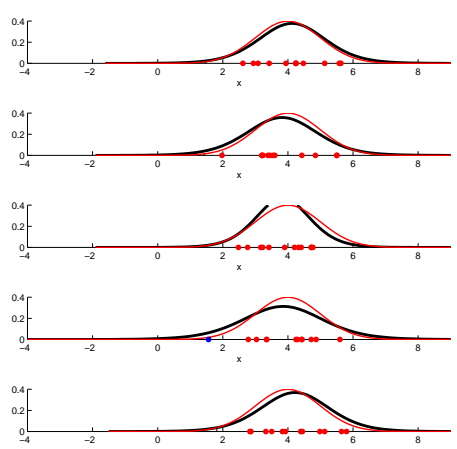

(d) $p\left(c_{\text {pre }} \mid \mathcal{X}_{k, \text { pre }}\right)$

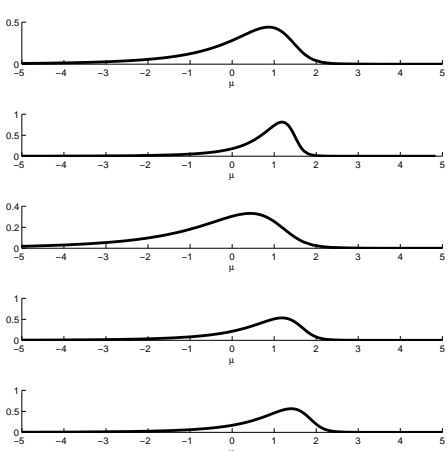

(b) $p\left(\mu_{1} \mid \mathcal{X}_{k, \text { post }}\right)$

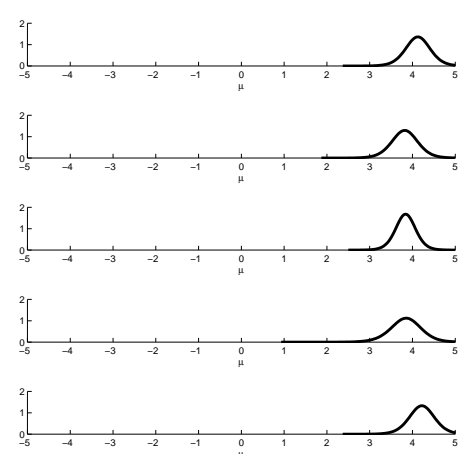

(e) $p\left(\mu_{1} \mid \mathcal{X}_{k, \text { pre }}\right)$

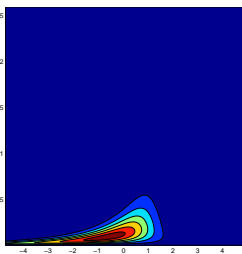

(g-3) $p\left(\mu, \beta \mid \mathcal{X}_{3, \text { post }}\right)$

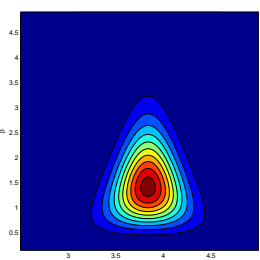

(h-3) $p\left(\mu, \beta \mid \mathcal{X}_{3, \text { pre }}\right)$

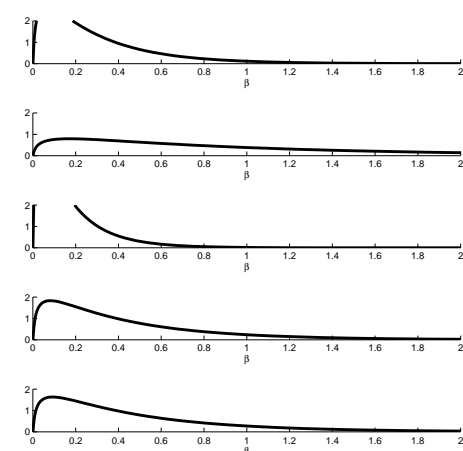

(c) $p\left(\beta_{1} \mid \mathcal{X}_{k, \text { post }}\right)$

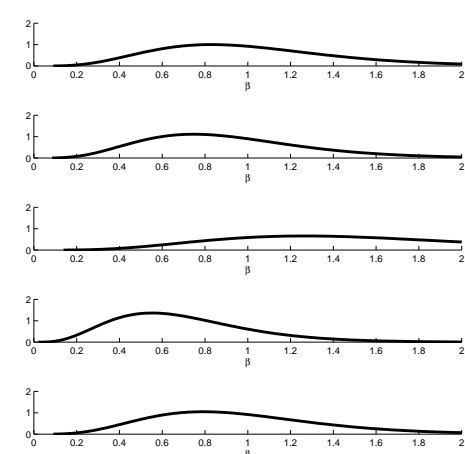

(f) $p\left(\beta_{1} \mid \mathcal{X}_{k, \text { pre }}\right)$

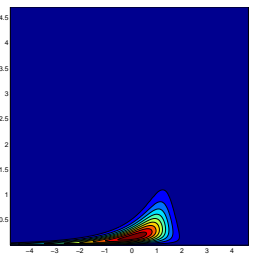

(g-1) $p\left(\mu, \beta \mid \mathcal{X}_{1, \text { post }}\right)$

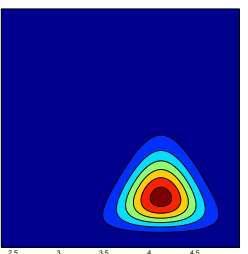

(h-1) $p\left(\mu, \beta \mid \mathcal{X}_{1, \text { pre }}\right)$

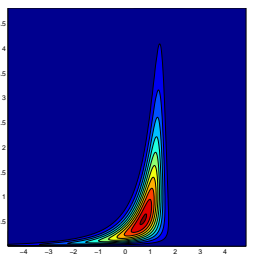

(g-2) $p\left(\mu, \beta \mid \mathcal{X}_{2, \text { post }}\right)$

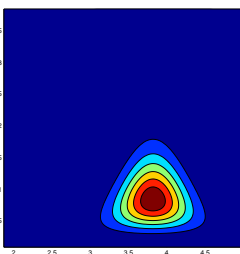

(h-2) $p\left(\mu, \beta \mid \mathcal{X}_{2, \text { pre }}\right)$

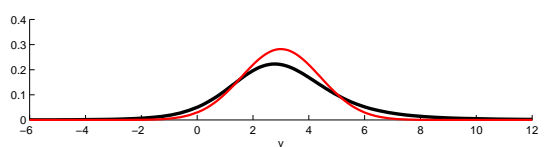

(i-2) $p\left(y \mid \mathcal{X}_{2, \text { pre }}, \mathcal{X}_{2, \text { post }}\right)$

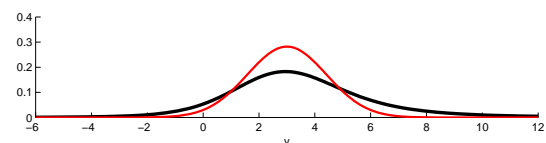

(i-5) $p\left(y \mid \mathcal{X}_{5, \text { pre }}, \mathcal{X}_{5, \text { post }}\right)$

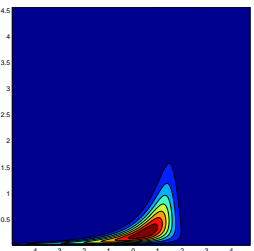

(g-4) $p\left(\mu, \beta \mid \mathcal{X}_{4, \text { post }}\right)$
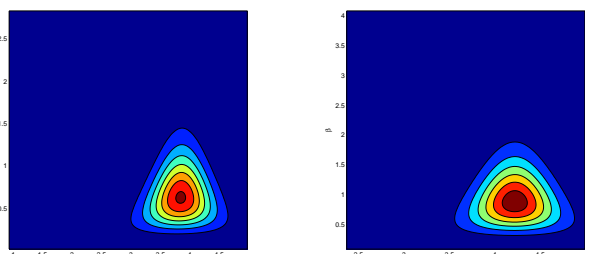

(h-4) $p\left(\mu, \beta \mid \mathcal{X}_{4, \text { pre }}\right)$

(h-5) $p\left(\mu, \beta \mid \mathcal{X}_{5, \text { pre }}\right)$

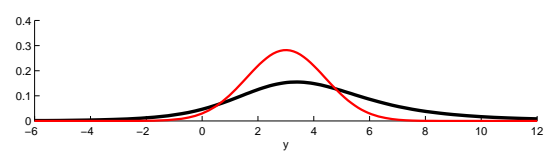

(i-1) $p\left(y \mid \mathcal{X}_{1, \text { pre }}, \mathcal{X}_{1, \text { post }}\right)$

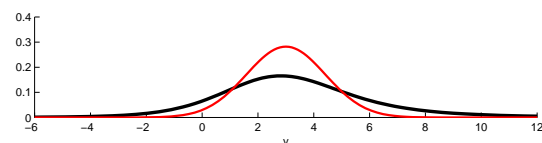

(i-4) $p\left(y \mid \mathcal{X}_{4, \text { pre }}, \mathcal{X}_{4, \text { post }}\right)$

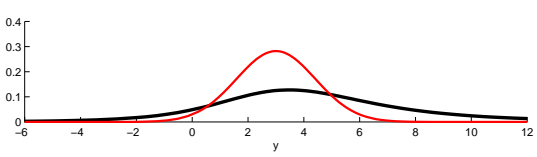

(i-3) $p\left(y \mid \mathcal{X}_{3, \text { pre }}, \mathcal{X}_{3, \text { post }}\right)$

Figure 4S: Bayesian inferences on five datasets, each of which contains $n_{v}=4$ detect data out of $n=12$ samples. 


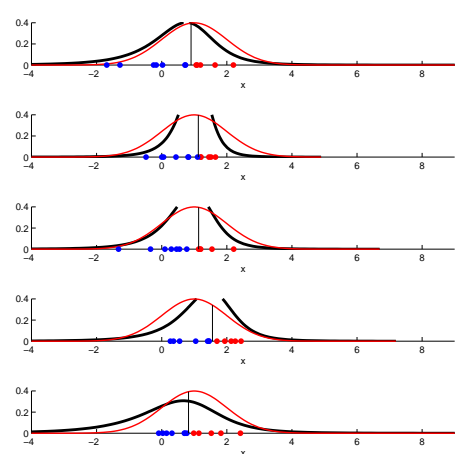

(a) $p\left(c_{\text {post }} \mid \mathcal{X}_{k \text {,post }}\right)$

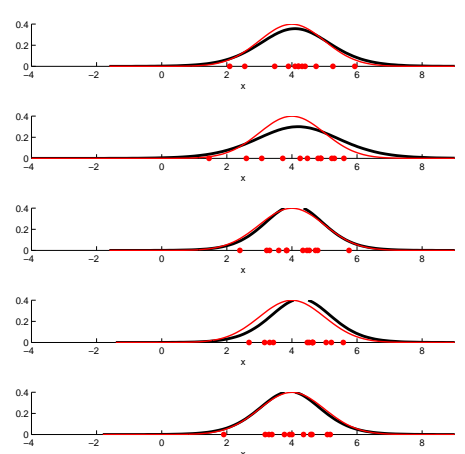

(d) $p\left(c_{\text {pre }} \mid \mathcal{X}_{k, \text { pre }}\right)$

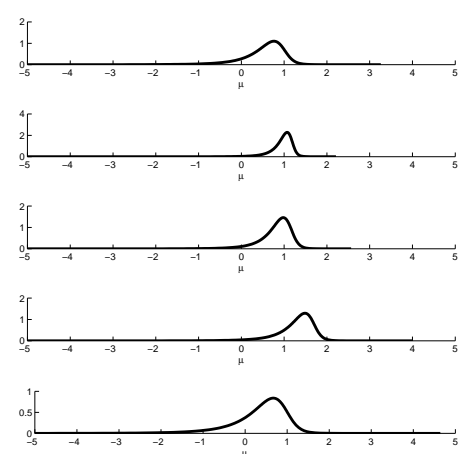

(b) $p\left(\mu_{1} \mid \mathcal{X}_{k, \text { post }}\right)$

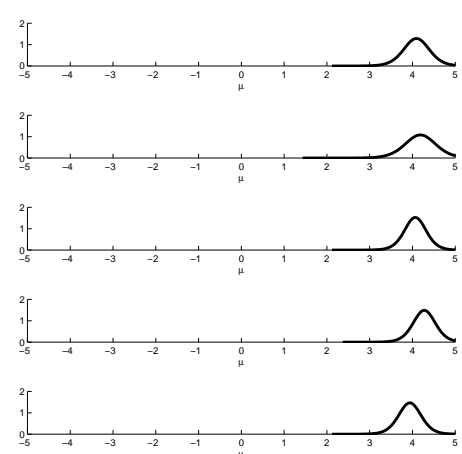

(e) $p\left(\mu_{1} \mid \mathcal{X}_{k, \text { pre }}\right)$

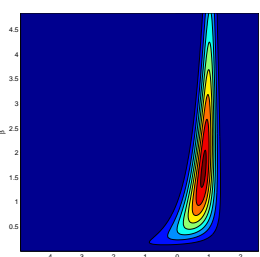

(g-3) $p\left(\mu, \beta \mid \mathcal{X}_{3, \text { post }}\right)$

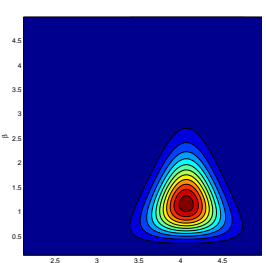

(h-3) $p\left(\mu, \beta \mid \mathcal{X}_{3, \text { pre }}\right)$

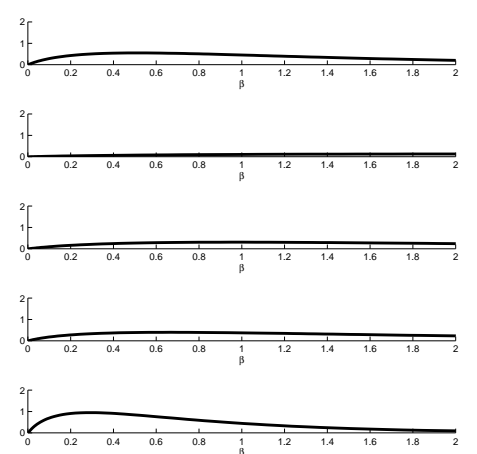

(c) $p\left(\beta_{1} \mid \mathcal{X}_{k, \text { post }}\right)$

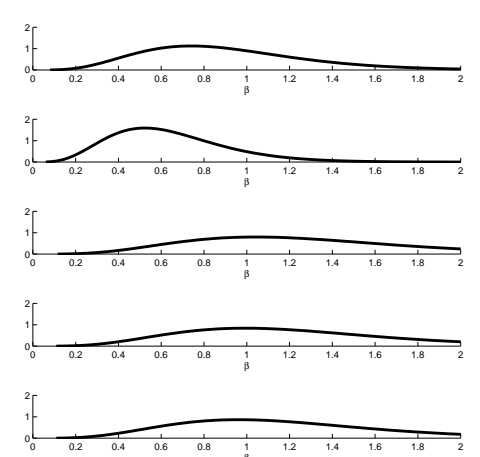

(f) $p\left(\beta_{1} \mid \mathcal{X}_{k, \text { pre }}\right)$

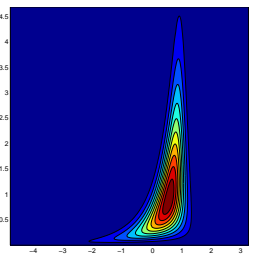

(g-1) $p\left(\mu, \beta \mid \mathcal{X}_{1, \text { post }}\right)$

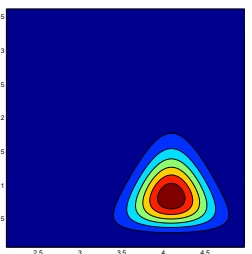

(h-1) $p\left(\mu, \beta \mid \mathcal{X}_{1, \text { pre }}\right)$

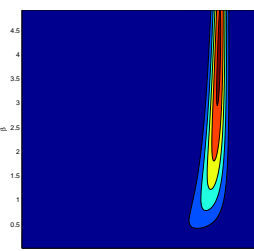

$(\mathrm{g}-2) p\left(\mu, \beta \mid \mathcal{X}_{2, \text { post }}\right)$

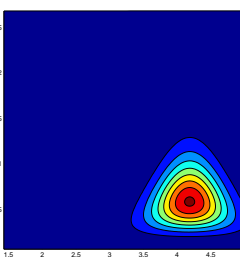

(h-2) $p\left(\mu, \beta \mid \mathcal{X}_{2, \text { pre }}\right)$

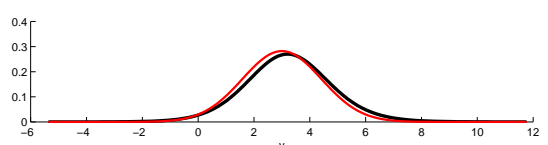

(i-2) $p\left(y \mid \mathcal{X}_{2, \text { pre }}, \mathcal{X}_{2, \text { post }}\right)$

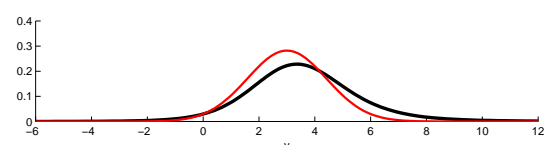

(i-5) $p\left(y \mid \mathcal{X}_{5, \text { pre }}, \mathcal{X}_{5, \text { post }}\right)$

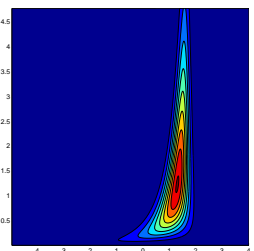

(g-4) $p\left(\mu, \beta \mid \mathcal{X}_{4, \text { post }}\right)$
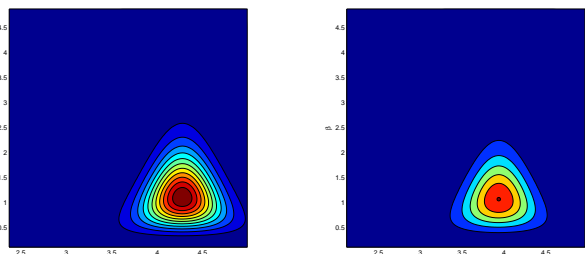

(h-4) $p\left(\mu, \beta \mid \mathcal{X}_{4, \text { pre }}\right)$

(h-5) $p\left(\mu, \beta \mid \mathcal{X}_{5, \text { pre }}\right)$

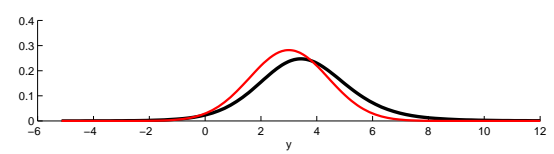

(i-1) $p\left(y \mid \mathcal{X}_{1, \text { pre }}, \mathcal{X}_{1, \text { post }}\right)$

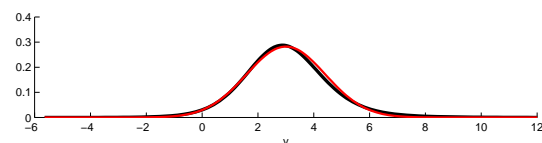

(i-4) $p\left(y \mid \mathcal{X}_{4, \text { pre }}, \mathcal{X}_{4, \text { post }}\right)$

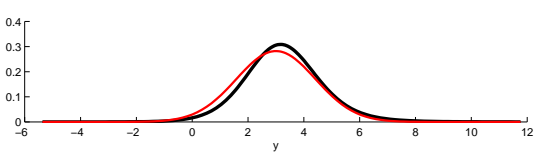

(i-3) $p\left(y \mid \mathcal{X}_{3, \text { pre }}, \mathcal{X}_{3, \text { post }}\right)$

Figure 5S: Bayesian inferences on five datasets, each of which contains $n_{v}=5$ detect data out of $n=12$ samples. 


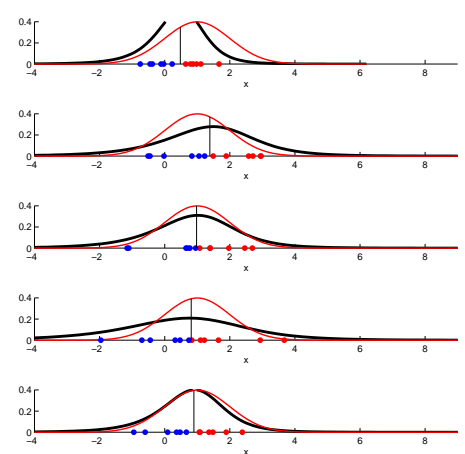

(a) $p\left(c_{\text {post }} \mid \mathcal{X}_{k \text {,post }}\right)$

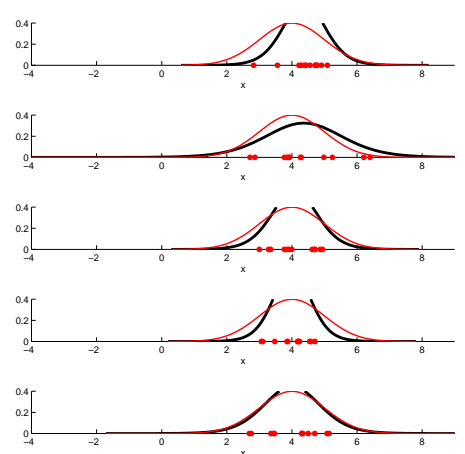

(d) $p\left(c_{\text {pre }} \mid \mathcal{X}_{k, \text { pre }}\right)$

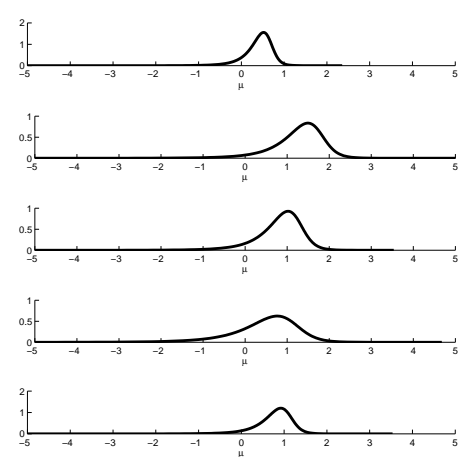

(b) $p\left(\mu_{1} \mid \mathcal{X}_{k, \text { post }}\right)$

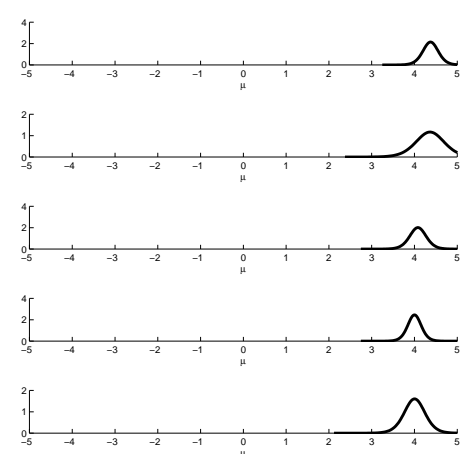

(e) $p\left(\mu_{1} \mid \mathcal{X}_{k, \text { pre }}\right)$

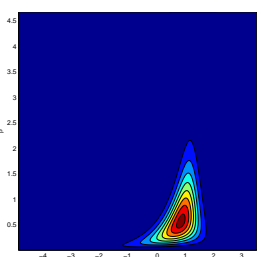

(g-3) $p\left(\mu, \beta \mid \mathcal{X}_{3, \text { post }}\right)$

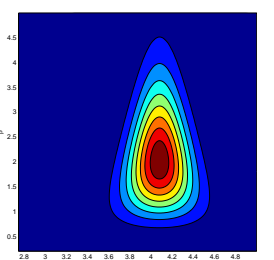

(h-3) $p\left(\mu, \beta \mid \mathcal{X}_{3, \text { pre }}\right)$

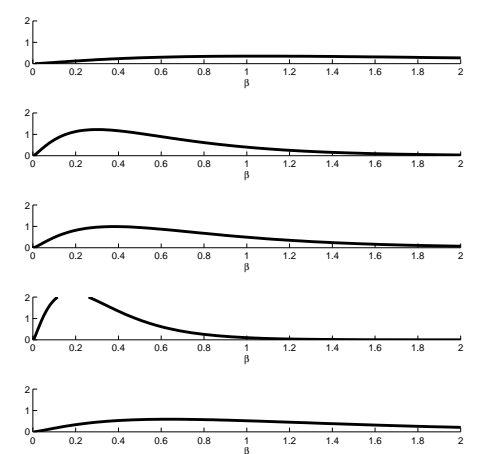

(c) $p\left(\beta_{1} \mid \mathcal{X}_{k, \text { post }}\right)$

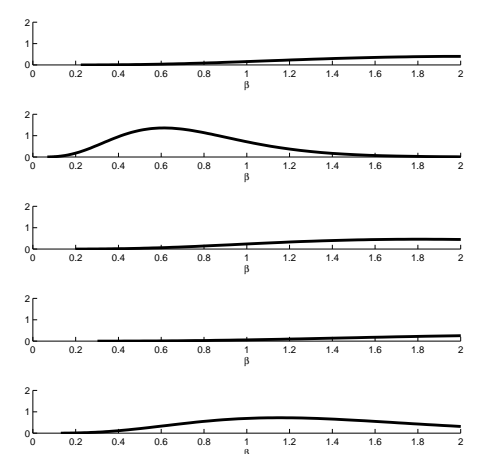

(f) $p\left(\beta_{1} \mid \mathcal{X}_{k, \text { pre }}\right)$

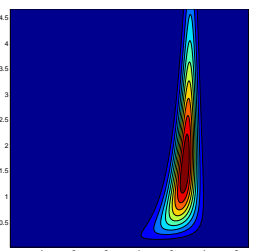

(g-1) $p\left(\mu, \beta \mid \mathcal{X}_{1, \text { post }}\right)$

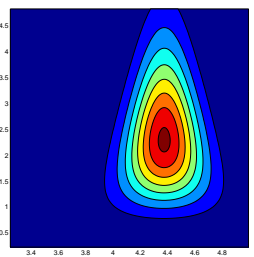

(h-1) $p\left(\mu, \beta \mid \mathcal{X}_{1, \text { pre }}\right)$

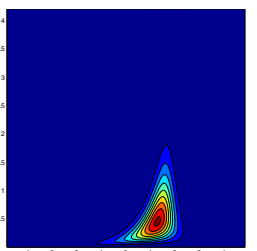

$(\mathrm{g}-2) p\left(\mu, \beta \mid \mathcal{X}_{2, \text { post }}\right)$

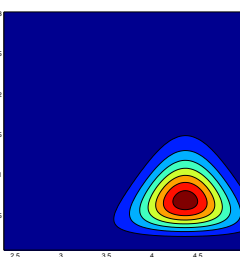

(h-2) $p\left(\mu, \beta \mid \mathcal{X}_{2, \text { pre }}\right)$

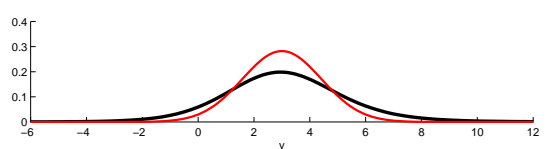

(i-2) $p\left(y \mid \mathcal{X}_{2, \text { pre }}, \mathcal{X}_{2, \text { post }}\right)$

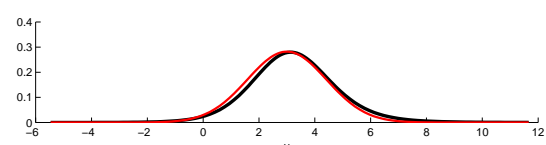

(i-5) $p\left(y \mid \mathcal{X}_{5, \text { pre }}, \mathcal{X}_{5, \text { post }}\right)$
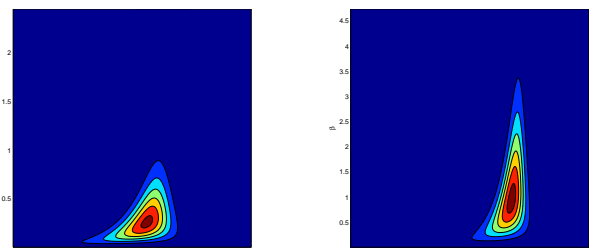

(g-4) $p\left(\mu, \beta \mid \mathcal{X}_{4, \text { post }}\right)$

$(\mathrm{g}-5) p\left(\mu, \beta \mid \mathcal{X}_{5, \text { post }}\right)$
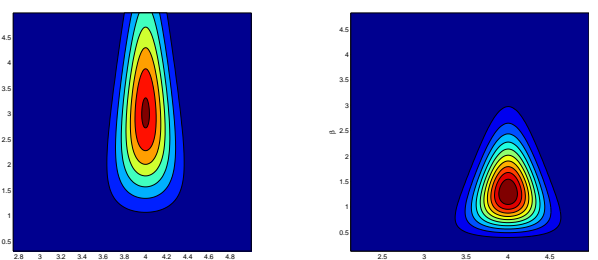

(h-4) $p\left(\mu, \beta \mid \mathcal{X}_{4, \text { pre }}\right)$

(h-5) $p\left(\mu, \beta \mid \mathcal{X}_{5, \text { pre }}\right)$

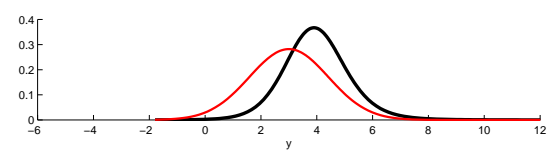

(i-1) $p\left(y \mid \mathcal{X}_{1, \text { pre }}, \mathcal{X}_{1, \text { post }}\right)$

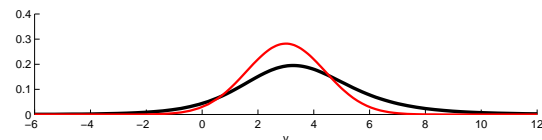

(i-4) $p\left(y \mid \mathcal{X}_{4, \text { pre }}, \mathcal{X}_{4, \text { post }}\right)$

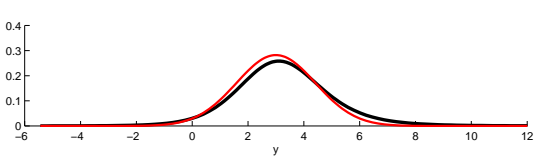

(i-3) $p\left(y \mid \mathcal{X}_{3, \text { pre }}, \mathcal{X}_{3, \text { post }}\right)$

Figure 6S: Bayesian inferences on five datasets, each of which contains $n_{v}=6$ detect data out of $n=12$ samples. 


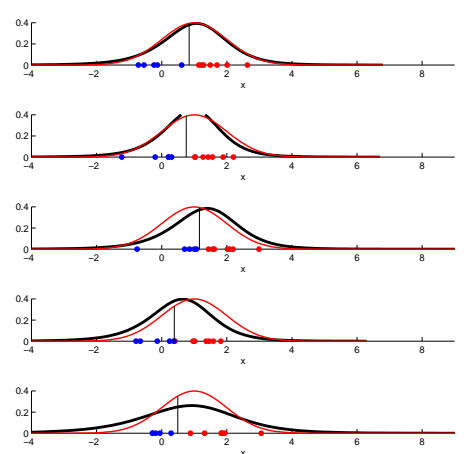

(a) $p\left(c_{\text {post }} \mid \mathcal{X}_{k, \text { post }}\right)$

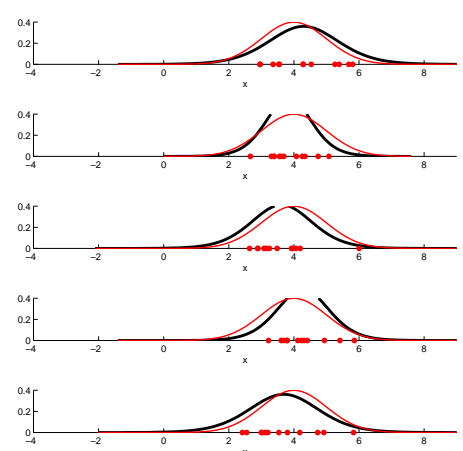

(d) $p\left(c_{\text {pre }} \mid \mathcal{X}_{k, \text { pre }}\right)$

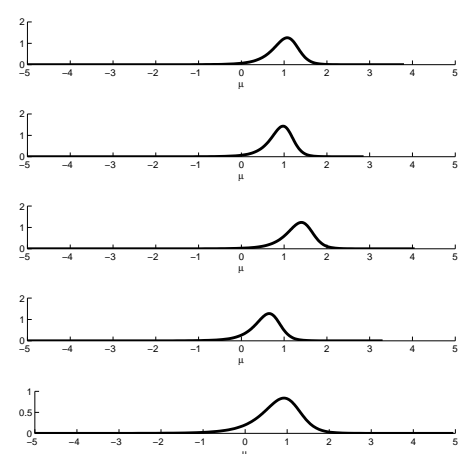

(b) $p\left(\mu_{1} \mid \mathcal{X}_{k, \text { post }}\right)$

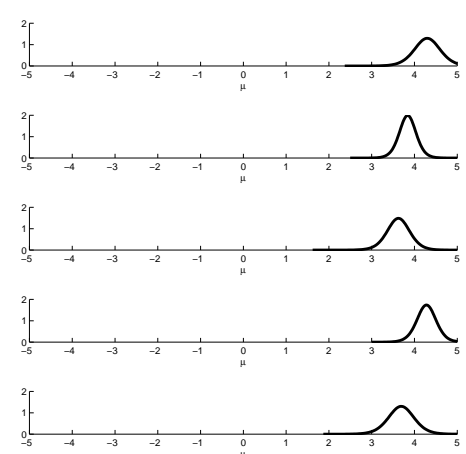

(e) $p\left(\mu_{1} \mid \mathcal{X}_{k, \text { pre }}\right)$

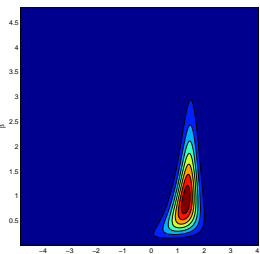

(g-3) $p\left(\mu, \beta \mid \mathcal{X}_{3, \text { post }}\right)$

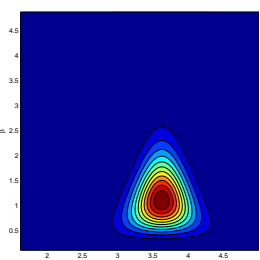

(h-3) $p\left(\mu, \beta \mid \mathcal{X}_{3, \text { pre }}\right)$

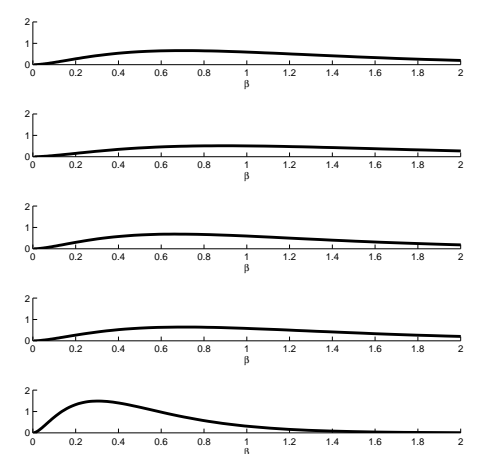

(c) $p\left(\beta_{1} \mid \mathcal{X}_{k, \text { post }}\right)$

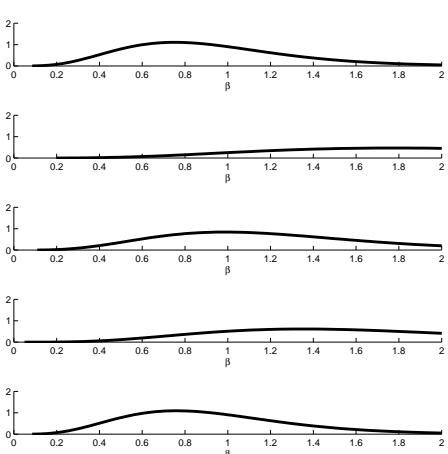

(f) $p\left(\beta_{1} \mid \mathcal{X}_{k, \text { pre }}\right)$

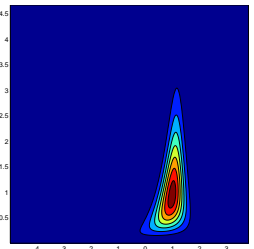

(g-1) $p\left(\mu, \beta \mid \mathcal{X}_{1, \text { post }}\right)$

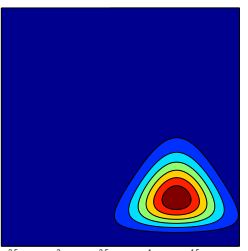

(h-1) $p\left(\mu, \beta \mid \mathcal{X}_{1, \text { pre }}\right)$

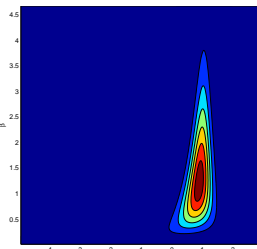

(g-2) $p\left(\mu, \beta \mid \mathcal{X}_{2, \text { post }}\right)$

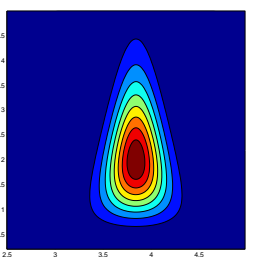

(h-2) $p\left(\mu, \beta \mid \mathcal{X}_{2, \text { pre }}\right)$

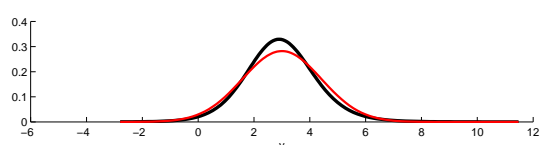

(i-2) $p\left(y \mid \mathcal{X}_{2, \text { pre }}, \mathcal{X}_{2, \text { post }}\right)$

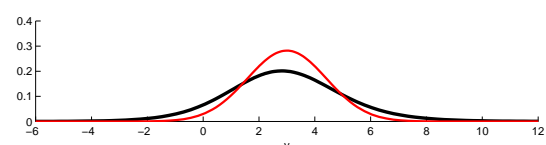

(i-5) $p\left(y \mid \mathcal{X}_{5, \text { pre }}, \mathcal{X}_{5, \text { post }}\right)$

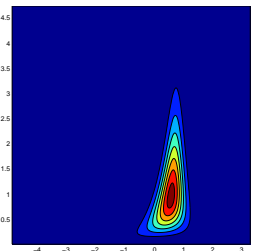

(g-4) $p\left(\mu, \beta \mid \mathcal{X}_{4, \text { post }}\right)$

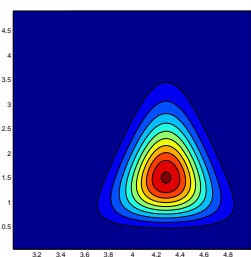

(h-4) $p\left(\mu, \beta \mid \mathcal{X}_{4, \text { pre }}\right)$

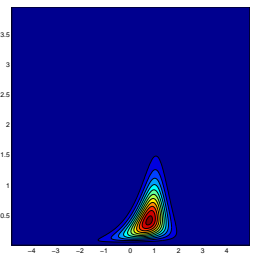

(g-5) $p\left(\mu, \beta \mid \mathcal{X}_{5, \text { post }}\right)$

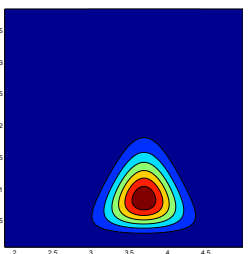

(h-5) $p\left(\mu, \beta \mid \mathcal{X}_{5, \text { pre }}\right)$

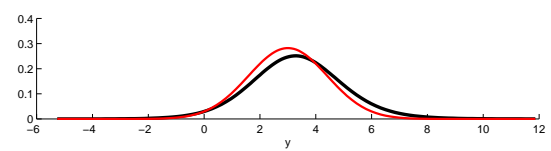

(i-1) $p\left(y \mid \mathcal{X}_{1, \text { pre }}, \mathcal{X}_{1, \text { post }}\right)$

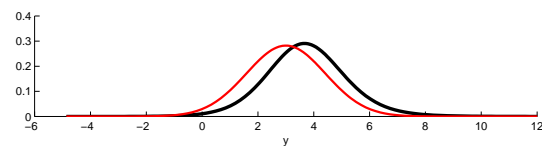

(i-4) $p\left(y \mid \mathcal{X}_{4, \text { pre }}, \mathcal{X}_{4, \text { post }}\right)$

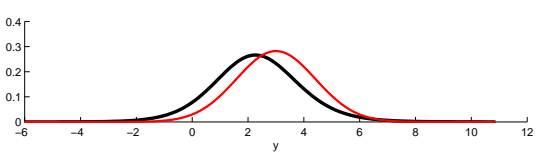

(i-3) $p\left(y \mid \mathcal{X}_{3, \text { pre }}, \mathcal{X}_{3, \text { post }}\right)$

Figure 7S: Bayesian inferences on five datasets, each of which contains $n_{v}=7$ detect data out of $n=12$ samples. 


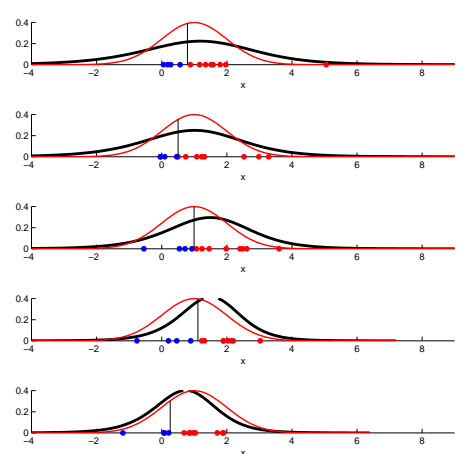

(a) $p\left(c_{\text {post }} \mid \mathcal{X}_{k, \text { post }}\right)$

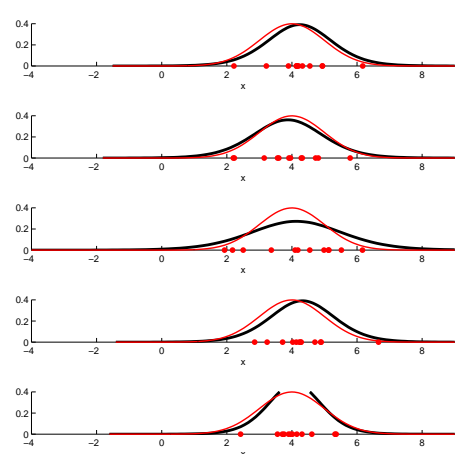

(d) $p\left(c_{\text {pre }} \mid \mathcal{X}_{k, \text { pre }}\right)$

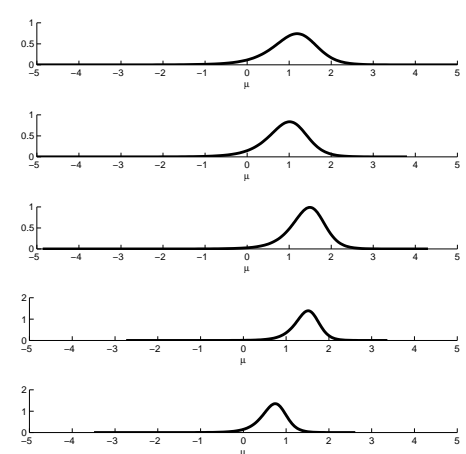

(b) $p\left(\mu_{1} \mid \mathcal{X}_{k, \text { post }}\right)$

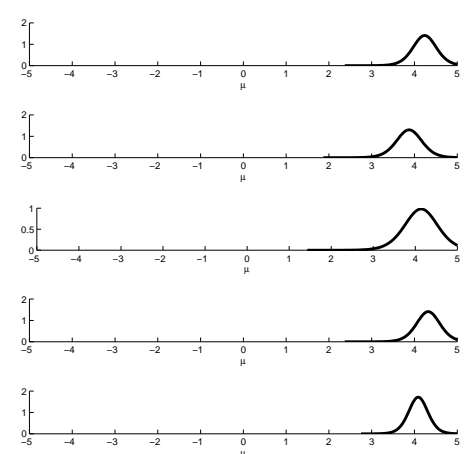

(e) $p\left(\mu_{1} \mid \mathcal{X}_{k, \text { pre }}\right)$

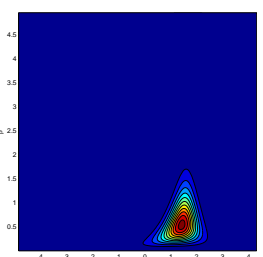

(g-3) $p\left(\mu, \beta \mid \mathcal{X}_{3, \text { post }}\right)$

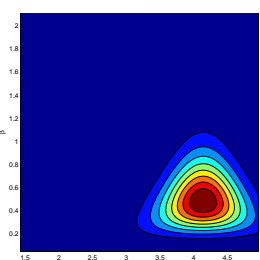

(h-3) $p\left(\mu, \beta \mid \mathcal{X}_{3, \text { pre }}\right)$

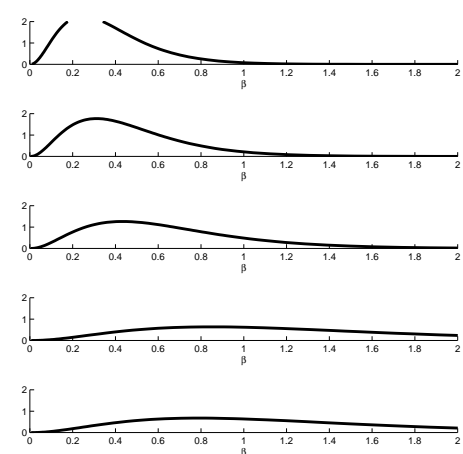

(c) $p\left(\beta_{1} \mid \mathcal{X}_{k, \text { post }}\right)$

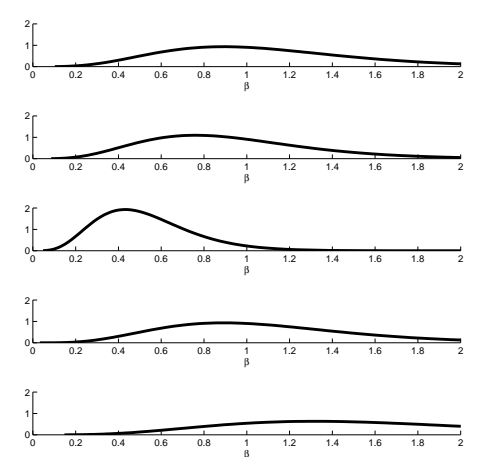

(f) $p\left(\beta_{1} \mid \mathcal{X}_{k, \text { pre }}\right)$

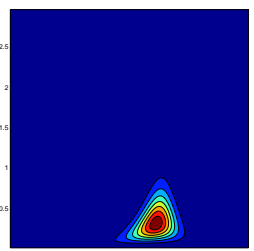

(g-1) $p\left(\mu, \beta \mid \mathcal{X}_{1, \text { post }}\right)$

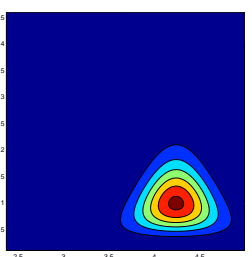

(h-1) $p\left(\mu, \beta \mid \mathcal{X}_{1, \text { pre }}\right)$

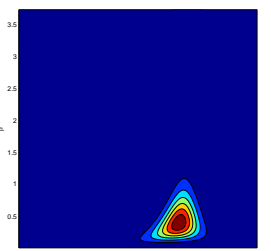

(g-2) $p\left(\mu, \beta \mid \mathcal{X}_{2, \text { post }}\right)$

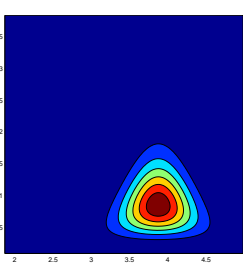

(h-2) $p\left(\mu, \beta \mid \mathcal{X}_{2, \text { pre }}\right)$

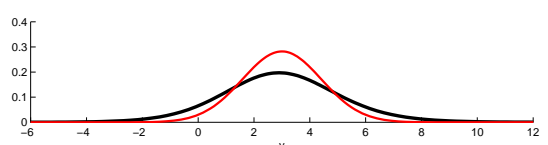

(i-2) $p\left(y \mid \mathcal{X}_{2, \text { pre }}, \mathcal{X}_{2, \text { post }}\right)$

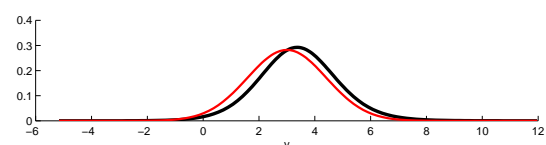

(i-5) $p\left(y \mid \mathcal{X}_{5, \text { pre }}, \mathcal{X}_{5, \text { post }}\right)$
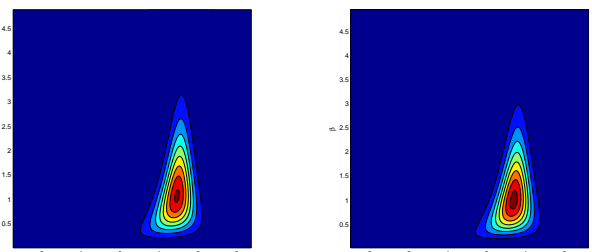

(g-4) $p\left(\mu, \beta \mid \mathcal{X}_{4, \text { post }}\right)$

$(\mathrm{g}-5) p\left(\mu, \beta \mid \mathcal{X}_{5, \text { post }}\right)$
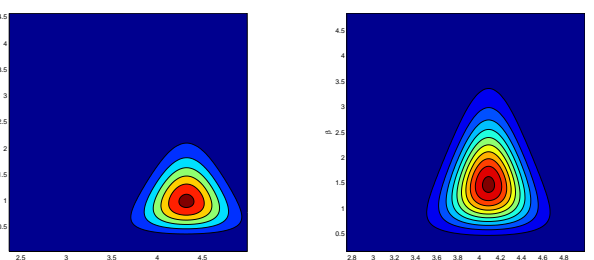

(h-4) $p\left(\mu, \beta \mid \mathcal{X}_{4, \text { pre }}\right)$

(h-5) $p\left(\mu, \beta \mid \mathcal{X}_{5, \text { pre }}\right)$

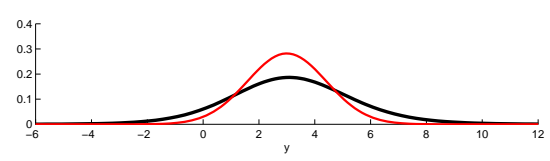

(i-1) $p\left(y \mid \mathcal{X}_{1, \text { pre }}, \mathcal{X}_{1, \text { post }}\right)$

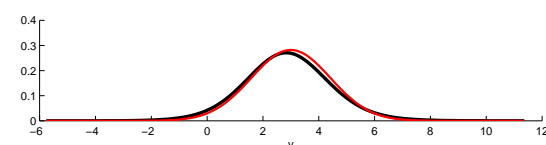

(i-4) $p\left(y \mid \mathcal{X}_{4, \text { pre }}, \mathcal{X}_{4, \text { post }}\right)$

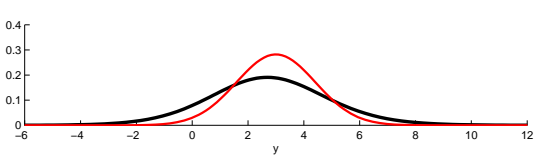

(i-3) $p\left(y \mid \mathcal{X}_{3, \text { pre }}, \mathcal{X}_{3, \text { post }}\right)$

Figure 8S: Bayesian inferences on five datasets, each of which contains $n_{v}=8$ detect data out of $n=12$ samples. 


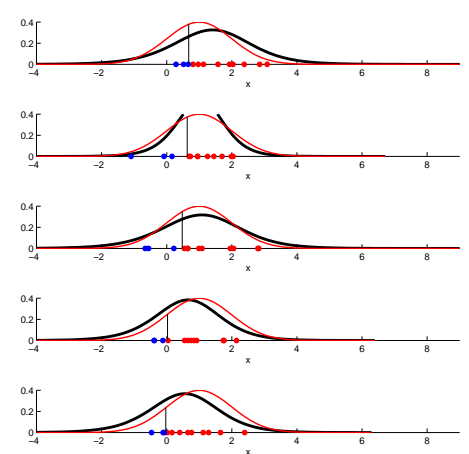

(a) $p\left(c_{\text {post }} \mid \mathcal{X}_{k, \text { post }}\right)$

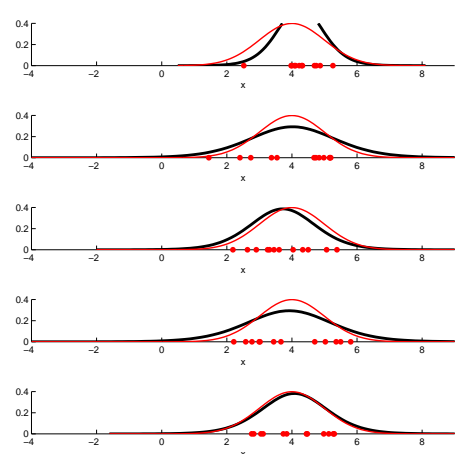

(d) $p\left(c_{\text {pre }} \mid \mathcal{X}_{k, \text { pre }}\right)$

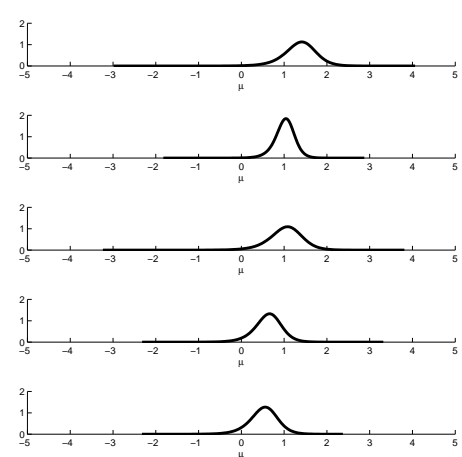

(b) $p\left(\mu_{1} \mid \mathcal{X}_{k, \text { post }}\right)$

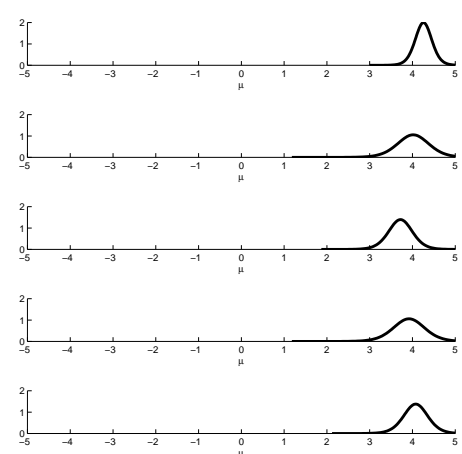

(e) $p\left(\mu_{1} \mid \mathcal{X}_{k, \text { pre }}\right)$

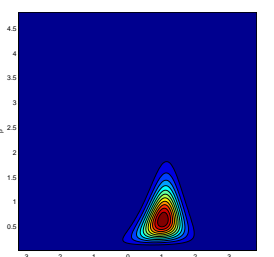

(g-3) $p\left(\mu, \beta \mid \mathcal{X}_{3, \text { post }}\right)$

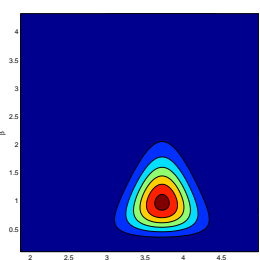

(h-3) $p\left(\mu, \beta \mid \mathcal{X}_{3, \text { pre }}\right)$

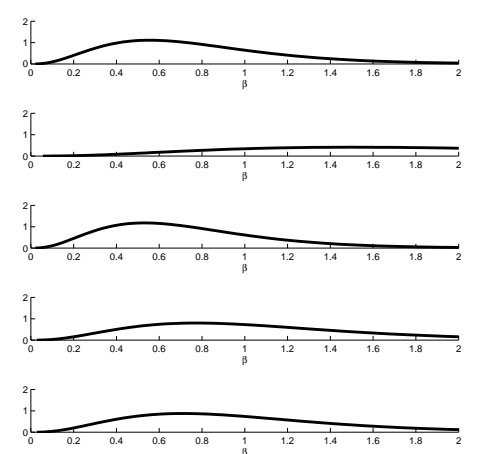

(c) $p\left(\beta_{1} \mid \mathcal{X}_{k, \text { post }}\right)$

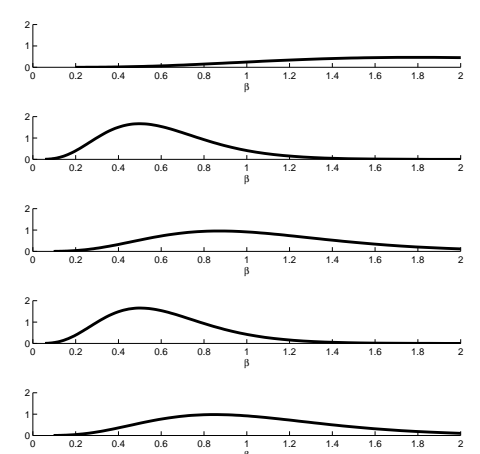

(f) $p\left(\beta_{1} \mid \mathcal{X}_{k, \text { pre }}\right)$

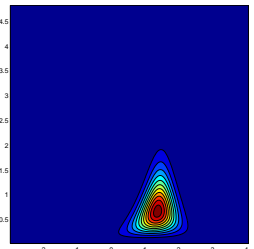

(g-1) $p\left(\mu, \beta \mid \mathcal{X}_{1, \text { post }}\right)$

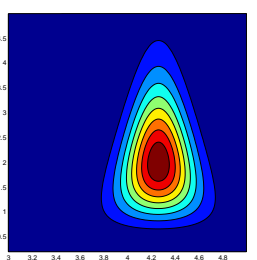

(h-1) $p\left(\mu, \beta \mid \mathcal{X}_{1, \text { pre }}\right)$

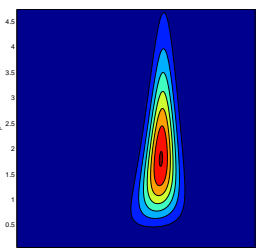

(g-2) $p\left(\mu, \beta \mid \mathcal{X}_{2, \text { post }}\right)$

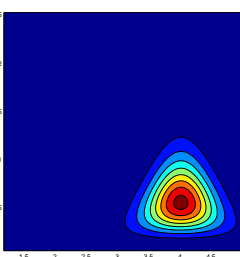

(h-2) $p\left(\mu, \beta \mid \mathcal{X}_{2, \text { pre }}\right)$

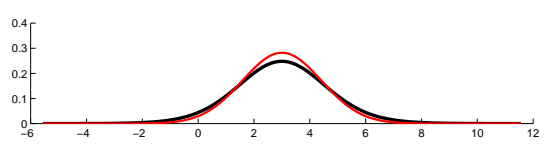

(i-2) $p\left(y \mid \mathcal{X}_{2, \text { pre }}, \mathcal{X}_{2, \text { post }}\right)$

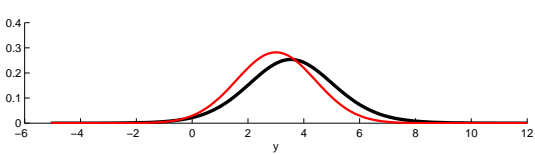

(i-5) $p\left(y \mid \mathcal{X}_{5, \text { pre }}, \mathcal{X}_{5, \text { post }}\right)$
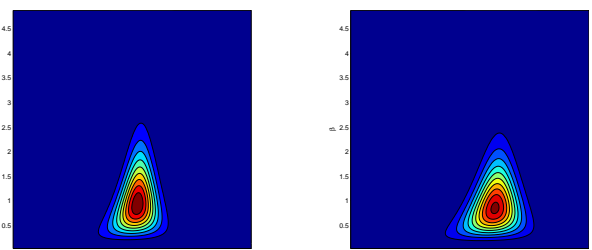

(g-4) $p\left(\mu, \beta \mid \mathcal{X}_{4, \text { post }}\right)$

(g-5) $p\left(\mu, \beta \mid \mathcal{X}_{5, \text { post }}\right)$
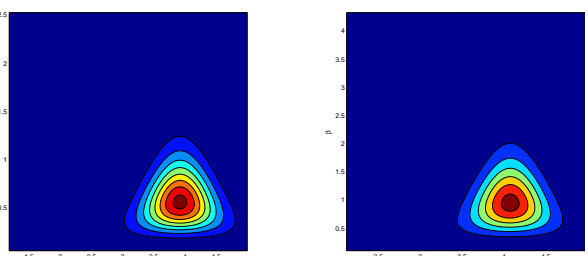

(h-4) $p\left(\mu, \beta \mid \mathcal{X}_{4, \text { pre }}\right)$

(h-5) $p\left(\mu, \beta \mid \mathcal{X}_{5, \text { pre }}\right)$

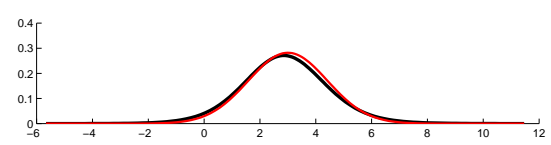

(i-1) $p\left(y \mid \mathcal{X}_{1, \text { pre }}, \mathcal{X}_{1, \text { post }}\right)$

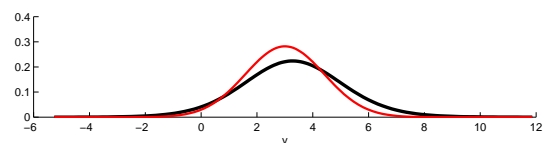

(i-4) $p\left(y \mid \mathcal{X}_{4, \text { pre }}, \mathcal{X}_{4, \text { post }}\right)$

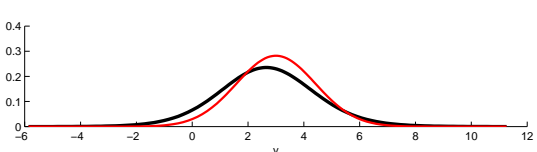

(i-3) $p\left(y \mid \mathcal{X}_{3, \text { pre }}, \mathcal{X}_{3, \text { post }}\right)$

Figure 9S: Bayesian inferences on five datasets, each of which contains $n_{v}=9$ detect data out of $n=12$ samples. 


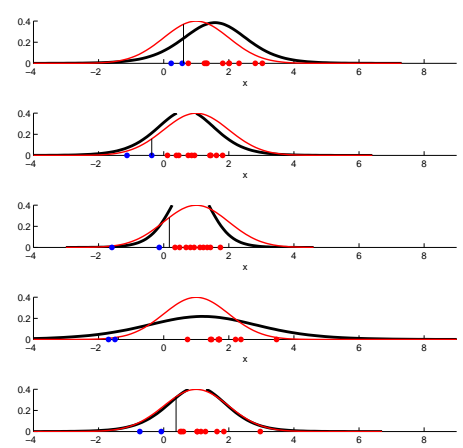

(a) $p\left(c_{\text {post }} \mid \mathcal{X}_{k, \text { post }}\right)$

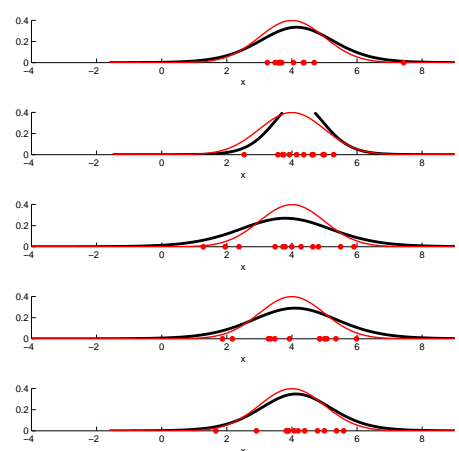

(d) $p\left(c_{\text {pre }} \mid \mathcal{X}_{k, \text { pre }}\right)$

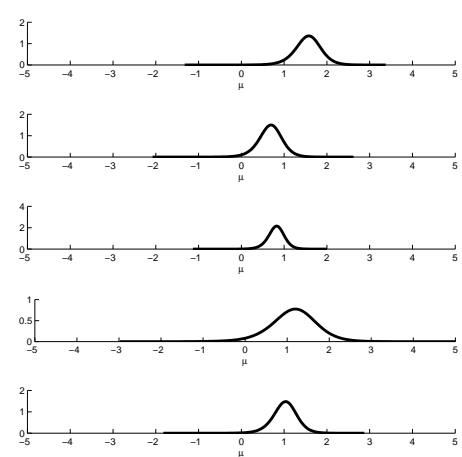

(b) $p\left(\mu_{1} \mid \mathcal{X}_{k, \text { post }}\right)$

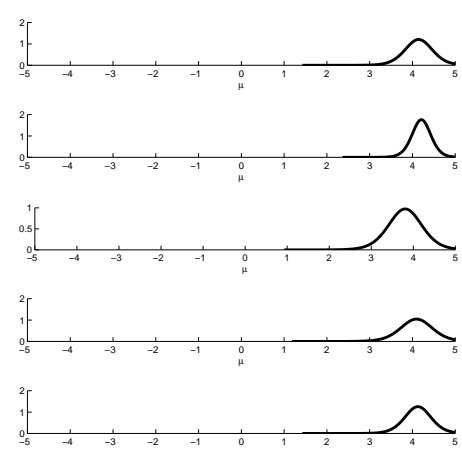

(e) $p\left(\mu_{1} \mid \mathcal{X}_{k, \text { pre }}\right)$

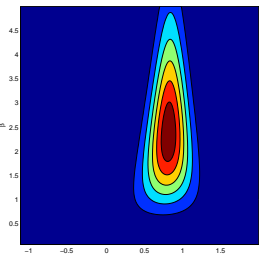

(g-3) $p\left(\mu, \beta \mid \mathcal{X}_{3, \text { post }}\right)$

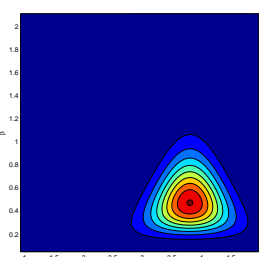

(h-3) $p\left(\mu, \beta \mid \mathcal{X}_{3, \text { pre }}\right)$

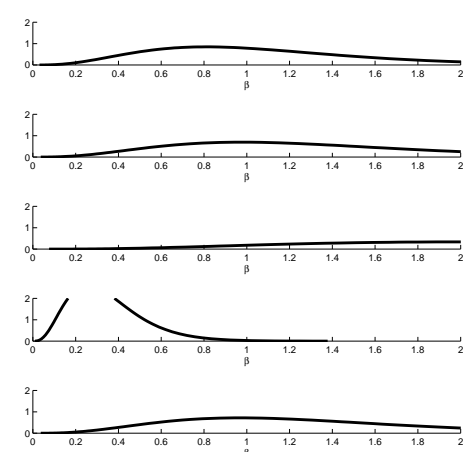

(c) $p\left(\beta_{1} \mid \mathcal{X}_{k, \text { post }}\right)$

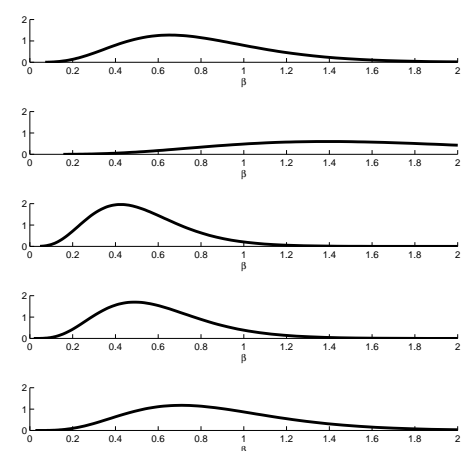

(f) $p\left(\beta_{1} \mid \mathcal{X}_{k, \text { pre }}\right)$

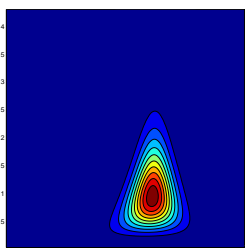

(g-1) $p\left(\mu, \beta \mid \mathcal{X}_{1, \text { post }}\right)$

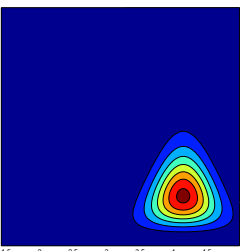

(h-1) $p\left(\mu, \beta \mid \mathcal{X}_{1, \text { pre }}\right)$

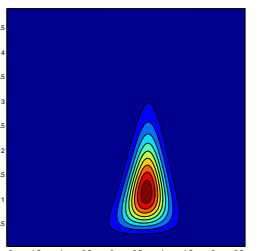

(g-2) $p\left(\mu, \beta \mid \mathcal{X}_{2, \text { post }}\right)$

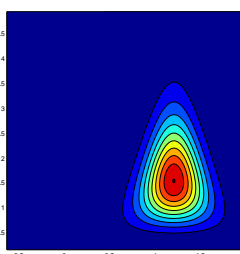

(h-2) $p\left(\mu, \beta \mid \mathcal{X}_{2, \text { pre }}\right)$

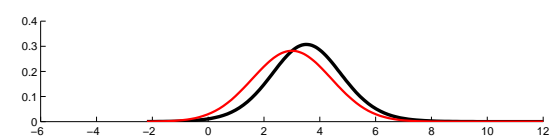

(i-2) $p\left(y \mid \mathcal{X}_{2, \text { pre }}, \mathcal{X}_{2, \text { post }}\right)$

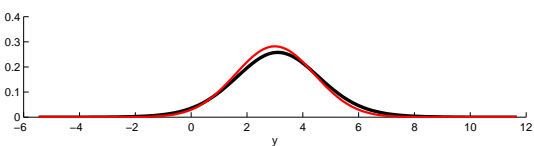

(i-5) $p\left(y \mid \mathcal{X}_{5, \text { pre }}, \mathcal{X}_{5, \text { post }}\right)$

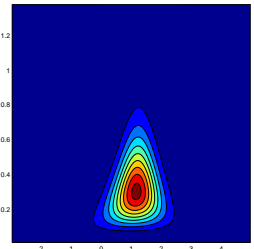

(g-4) $p\left(\mu, \beta \mid \mathcal{X}_{4, \text { post }}\right)$

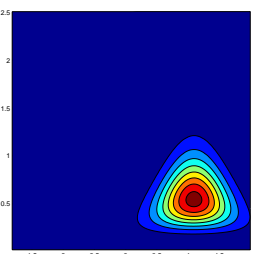

(h-4) $p\left(\mu, \beta \mid \mathcal{X}_{4, \text { pre }}\right)$

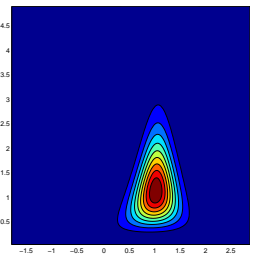

(g-5) $p\left(\mu, \beta \mid \mathcal{X}_{5, \text { post }}\right)$

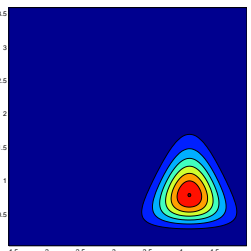

(h-5) $p\left(\mu, \beta \mid \mathcal{X}_{5, \text { pre }}\right)$

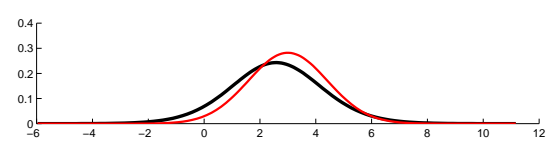

(i-1) $p\left(y \mid \mathcal{X}_{1, \text { pre }}, \mathcal{X}_{1, \text { post }}\right)$

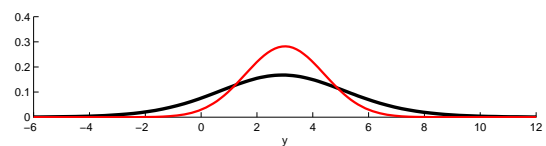

(i-4) $p\left(y \mid \mathcal{X}_{4, \text { pre }}, \mathcal{X}_{4, \text { post }}\right)$

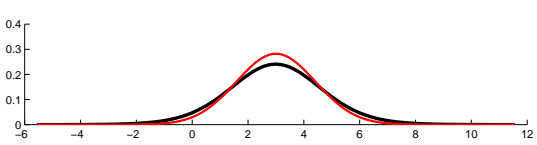

$(\mathrm{i}-3) p\left(y \mid \mathcal{X}_{3, \text { pre }}, \mathcal{X}_{3, \text { post }}\right)$

Figure 10S: Bayesian inferences on five datasets, each of which contains $n_{v}=10$ detect data out of $n=12$ samples. 


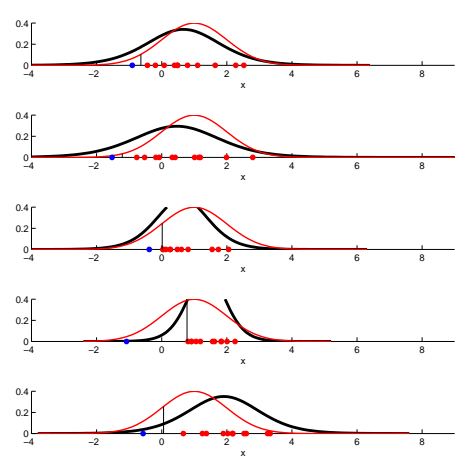

(a) $p\left(c_{\text {post }} \mid \mathcal{X}_{k, \text { post }}\right)$

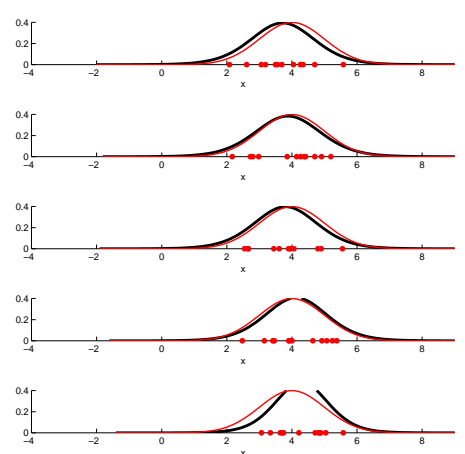

(d) $p\left(c_{\text {pre }} \mid \mathcal{X}_{k, \text { pre }}\right)$

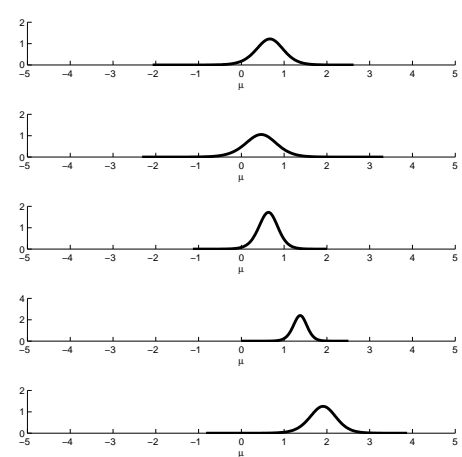

(b) $p\left(\mu_{1} \mid \mathcal{X}_{k, \text { post }}\right)$

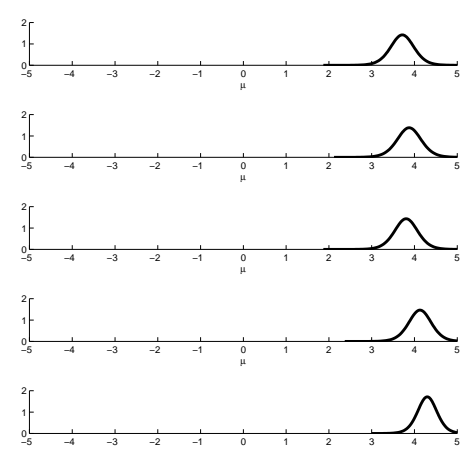

(e) $p\left(\mu_{1} \mid \mathcal{X}_{k, \text { pre }}\right)$

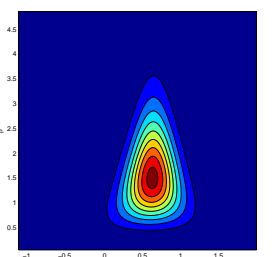

$(\mathrm{g}-3) p\left(\mu, \beta \mid \mathcal{X}_{3, \text { post }}\right)$

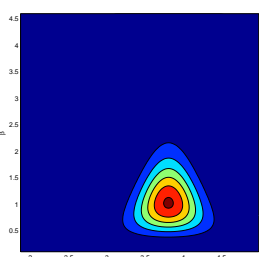

(h-3) $p\left(\mu, \beta \mid \mathcal{X}_{3, \text { pre }}\right)$

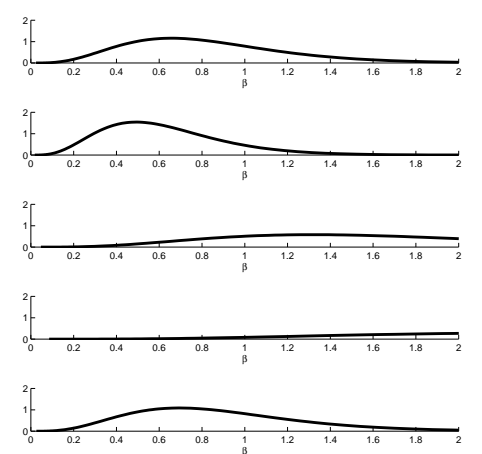

(c) $p\left(\beta_{1} \mid \mathcal{X}_{k, \text { post }}\right)$

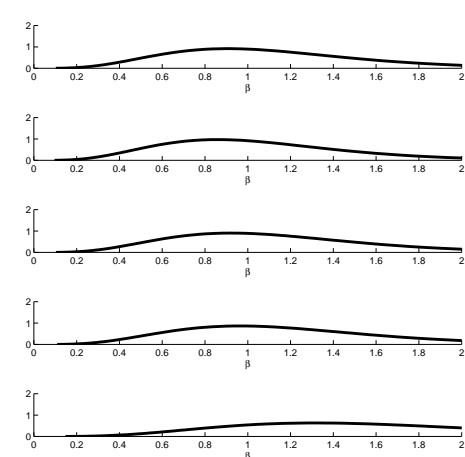

(f) $p\left(\beta_{1} \mid \mathcal{X}_{k, \text { pre }}\right)$

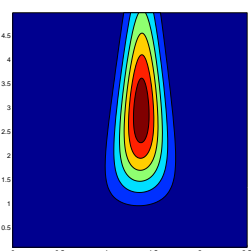

(g-4) $p\left(\mu, \beta \mid \mathcal{X}_{4, \text { post }}\right)$

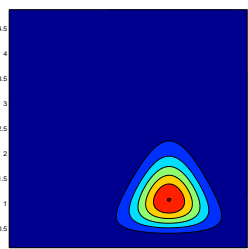

(h-4) $p\left(\mu, \beta \mid \mathcal{X}_{4, \text { pre }}\right)$

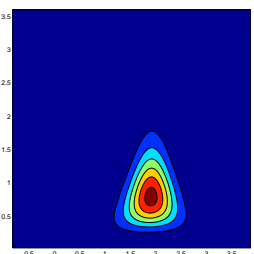

$(\mathrm{g}-5) p\left(\mu, \beta \mid \mathcal{X}_{5, \text { post }}\right)$

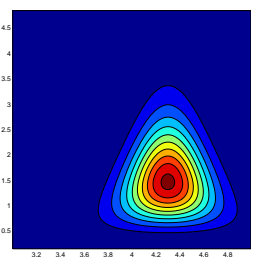

(h-5) $p\left(\mu, \beta \mid \mathcal{X}_{5, \text { pre }}\right)$

(h-1) $p\left(\mu, \beta \mid \mathcal{X}_{1, \text { pre }}\right)$

(h-2) $p\left(\mu, \beta \mid \mathcal{X}_{2, \text { pre }}\right)$

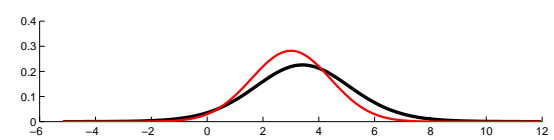

(i-2) $p\left(y \mid \mathcal{X}_{2, \text { pre }}, \mathcal{X}_{2, \text { post }}\right)$

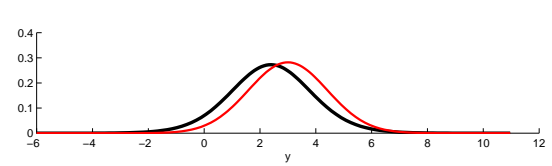

(i-5) $p\left(y \mid \mathcal{X}_{5, \text { pre }}, \mathcal{X}_{5, \text { post }}\right)$

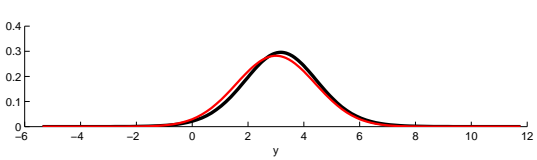

(i-3) $p\left(y \mid \mathcal{X}_{3, \text { pre }}, \mathcal{X}_{3, \text { post }}\right)$

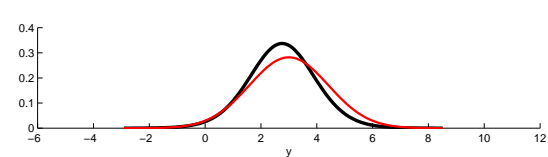

(i-4) $p\left(y \mid \mathcal{X}_{4, \text { pre }}, \mathcal{X}_{4, \text { post }}\right)$

Figure 11S: Bayesian inferences on five datasets, each of which contains $n_{v}=11$ detect data out of $n=12$ samples. 


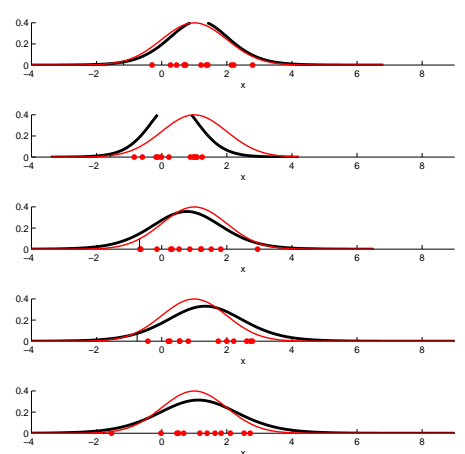

(a) $p\left(c_{\text {post }} \mid \mathcal{X}_{k, \text { post }}\right)$

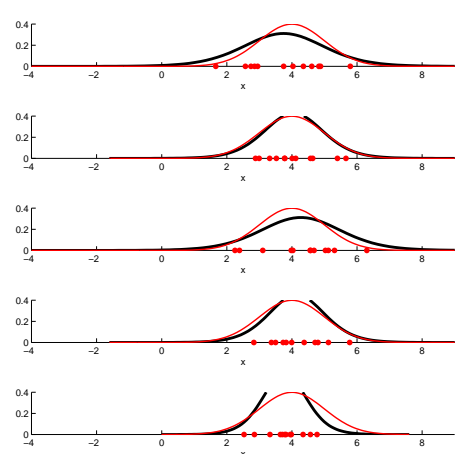

(d) $p\left(c_{\text {pre }} \mid \mathcal{X}_{k, \text { pre }}\right)$

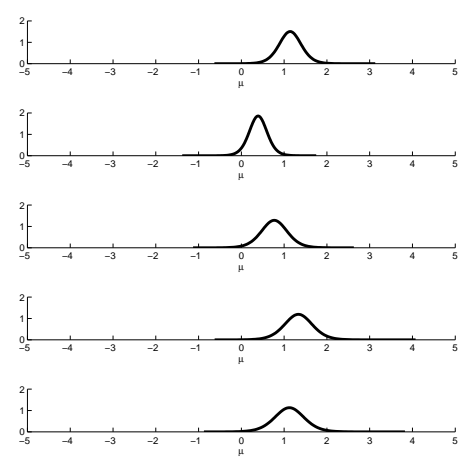

(b) $p\left(\mu_{1} \mid \mathcal{X}_{k, \text { post }}\right)$

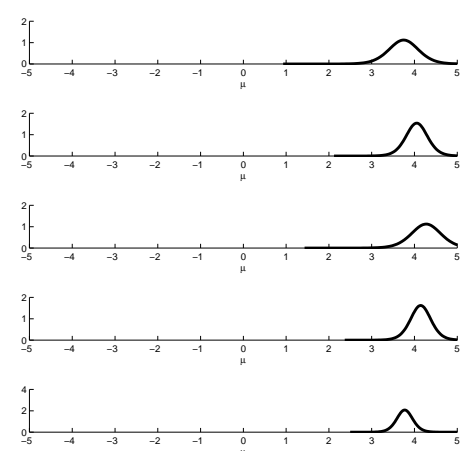

(e) $p\left(\mu_{1} \mid \mathcal{X}_{k, \text { pre }}\right)$

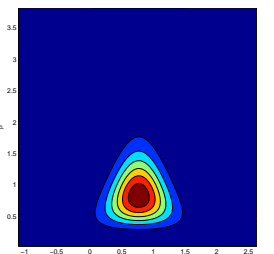

(g-3) $p\left(\mu, \beta \mid \mathcal{X}_{3, \text { post }}\right)$

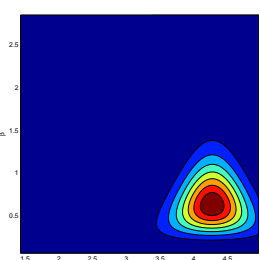

(h-3) $p\left(\mu, \beta \mid \mathcal{X}_{3, \text { pre }}\right)$

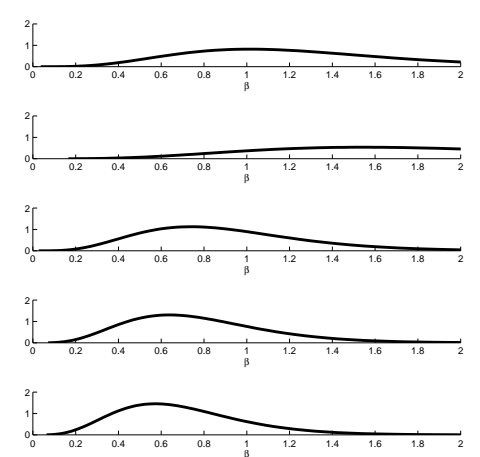

(c) $p\left(\beta_{1} \mid \mathcal{X}_{k, \text { post }}\right)$

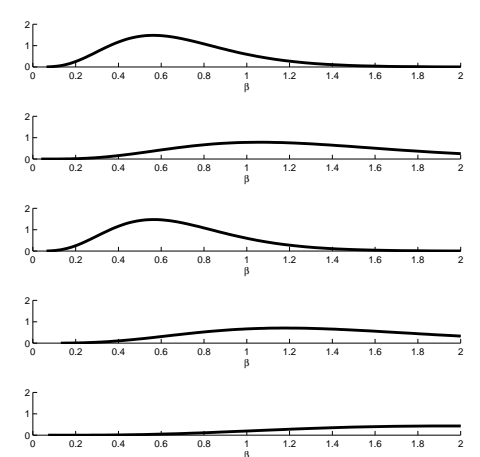

(f) $p\left(\beta_{1} \mid \mathcal{X}_{k, \text { pre }}\right)$

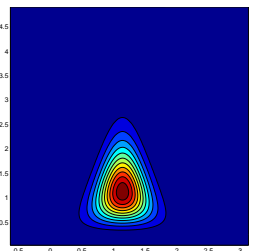

(g-1) $p\left(\mu, \beta \mid \mathcal{X}_{1, \text { post }}\right)$

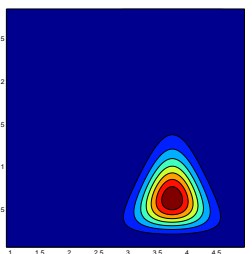

(h-1) $p\left(\mu, \beta \mid \mathcal{X}_{1, \text { pre }}\right)$

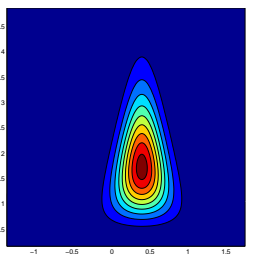

(g-2) $p\left(\mu, \beta \mid \mathcal{X}_{2, \text { post }}\right)$

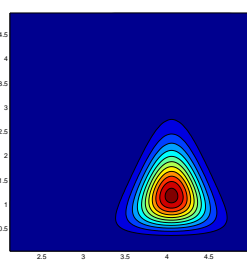

(h-2) $p\left(\mu, \beta \mid \mathcal{X}_{2, \text { pre }}\right)$

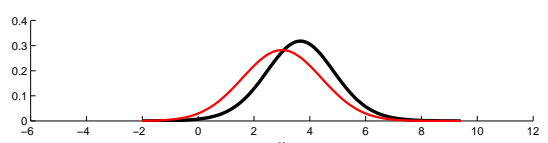

(i-2) $p\left(y \mid \mathcal{X}_{2, \text { pre }}, \mathcal{X}_{2, \text { post }}\right)$

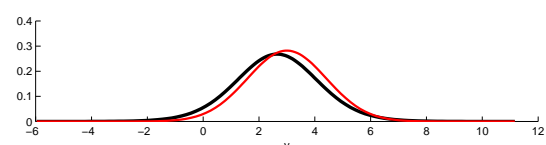

(i-5) $p\left(y \mid \mathcal{X}_{5, \text { pre }}, \mathcal{X}_{5, \text { post }}\right)$

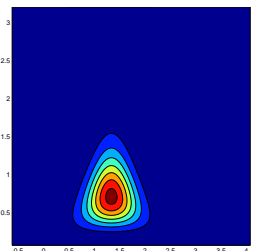

(g-4) $p\left(\mu, \beta \mid \mathcal{X}_{4, \text { post }}\right)$

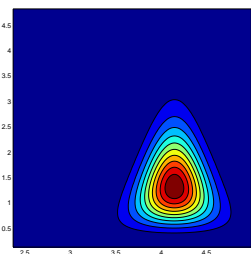

(h-4) $p\left(\mu, \beta \mid \mathcal{X}_{4, \text { pre }}\right)$

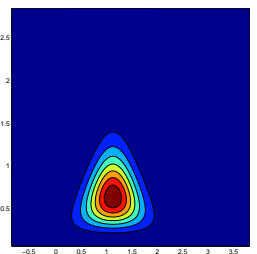

(g-5) $p\left(\mu, \beta \mid \mathcal{X}_{5, \text { post }}\right)$

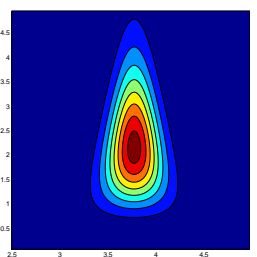

(h-5) $p\left(\mu, \beta \mid \mathcal{X}_{5, \text { pre }}\right)$

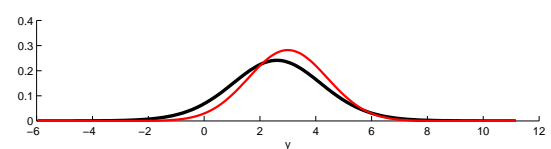

(i-1) $p\left(y \mid \mathcal{X}_{1, \text { pre }}, \mathcal{X}_{1, \text { post }}\right)$

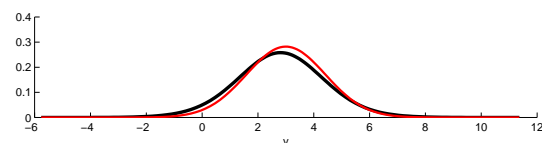

(i-4) $p\left(y \mid \mathcal{X}_{4, \text { pre }}, \mathcal{X}_{4, \text { post }}\right)$

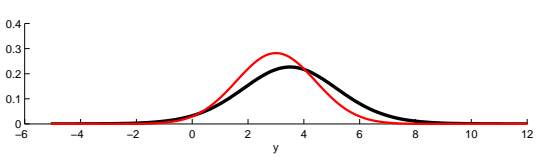

(i-3) $p\left(y \mid \mathcal{X}_{3, \text { pre }}, \mathcal{X}_{3, \text { post }}\right)$

Figure 12S: Bayesian inferences on five datasets, each of which contains $n_{v}=12$ detect data out of $n=12$ samples. 


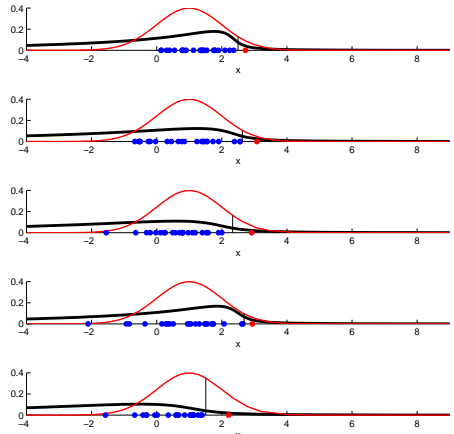

(a) $p\left(c_{\text {post }} \mid \mathcal{X}_{k, \text { post }}\right)$

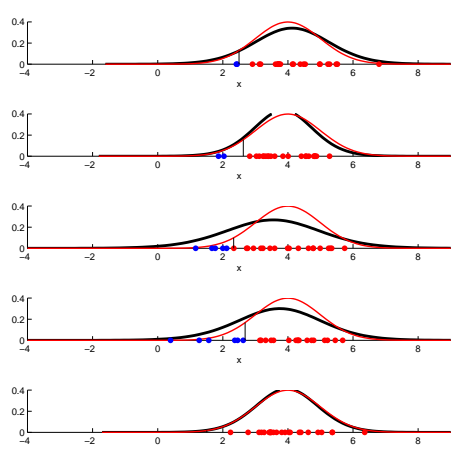

(d) $p\left(c_{\text {pre }} \mid \mathcal{X}_{k, \text { pre }}\right)$

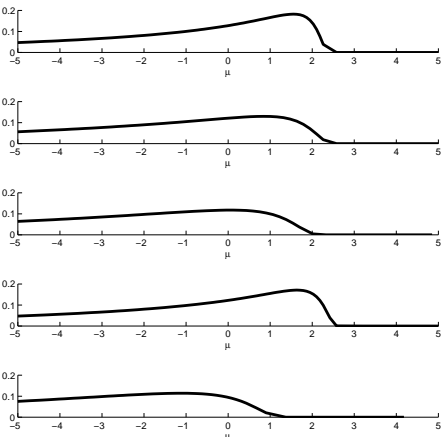

(b) $p\left(\mu_{1} \mid \mathcal{X}_{k, \text { post }}\right)$

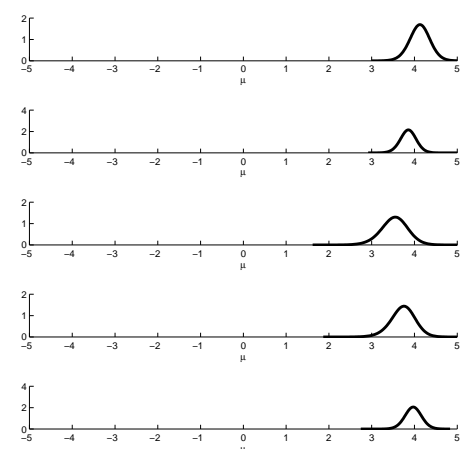

(e) $p\left(\mu_{1} \mid \mathcal{X}_{k, \text { pre }}\right)$

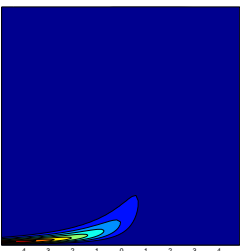

(g-3) $p\left(\mu, \beta \mid \mathcal{X}_{3, \text { post }}\right)$

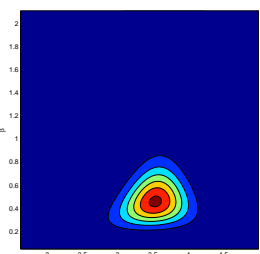

(h-3) $p\left(\mu, \beta \mid \mathcal{X}_{3, \text { pre }}\right)$

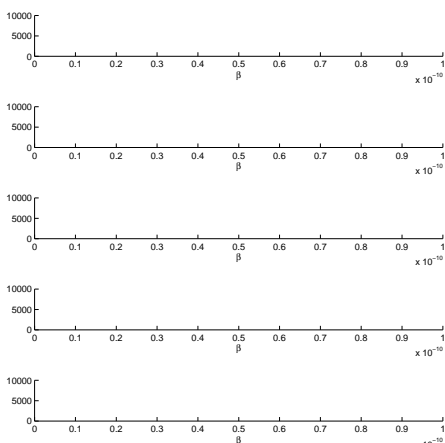

(c) $p\left(\beta_{1} \mid \mathcal{X}_{k, \text { post }}\right)$

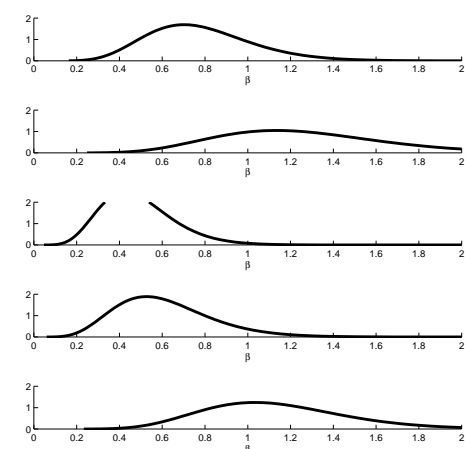

(f) $p\left(\beta_{1} \mid \mathcal{X}_{k, \text { pre }}\right)$

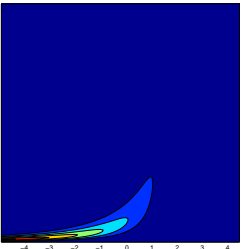

(g-1) $p\left(\mu, \beta \mid \mathcal{X}_{1, \text { post }}\right)$

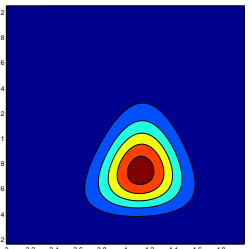

(h-1) $p\left(\mu, \beta \mid \mathcal{X}_{1, \text { pre }}\right)$

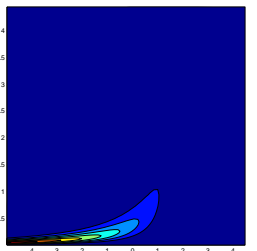

(g-2) $p\left(\mu, \beta \mid \mathcal{X}_{2, \text { post }}\right)$

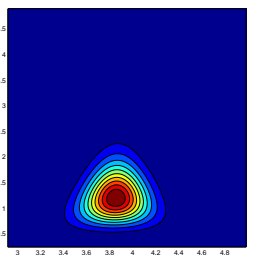

(h-2) $p\left(\mu, \beta \mid \mathcal{X}_{2, \text { pre }}\right)$

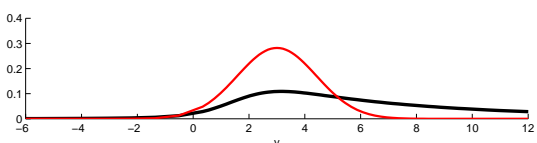

(i-2) $p\left(y \mid \mathcal{X}_{2, \text { pre }}, \mathcal{X}_{2, \text { post }}\right)$

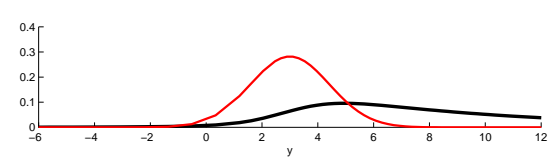

(i-5) $p\left(y \mid \mathcal{X}_{5, \text { pre }}, \mathcal{X}_{5, \text { post }}\right)$
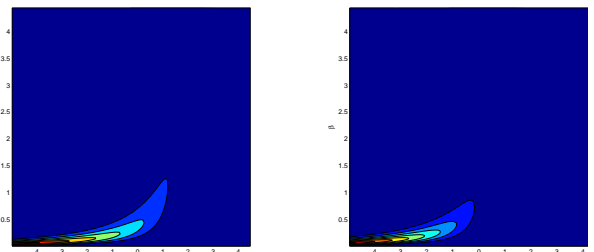

(g-4) $p\left(\mu, \beta \mid \mathcal{X}_{4, \text { post }}\right)$

(g-5) $p\left(\mu, \beta \mid \mathcal{X}_{5, \text { post }}\right)$
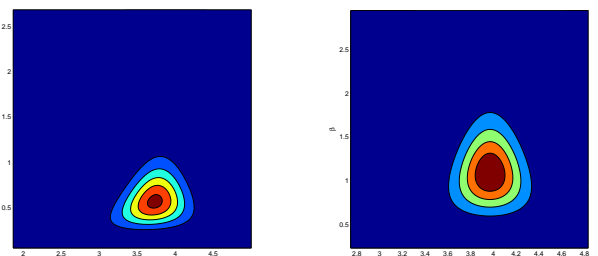

(h-4) $p\left(\mu, \beta \mid \mathcal{X}_{4, \text { pre }}\right)$

(h-5) $p\left(\mu, \beta \mid \mathcal{X}_{5, \text { pre }}\right)$

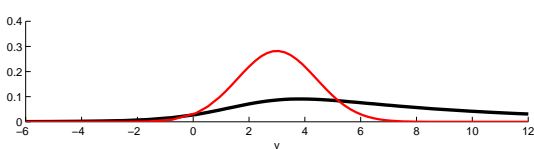

(i-3) $p\left(y \mid \mathcal{X}_{3, \text { pre }}, \mathcal{X}_{3, \text { post }}\right)$

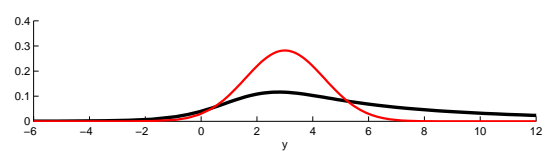

(i-4) $p\left(y \mid \mathcal{X}_{4, \text { pre }}, \mathcal{X}_{4, \text { post }}\right)$

Figure 13S: Bayesian inferences on five datasets, each of which contains $n_{v}=1$ detect data out of $n=24$ samples. 


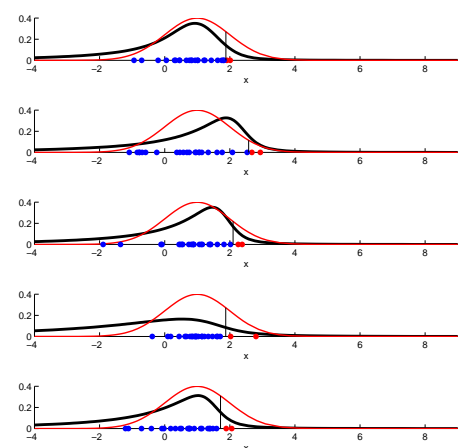

(a) $p\left(c_{\text {post }} \mid \mathcal{X}_{k, \text { post }}\right)$

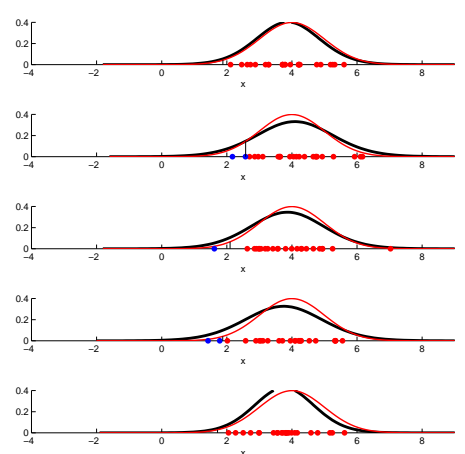

(d) $p\left(c_{\text {pre }} \mid \mathcal{X}_{k, \text { pre }}\right)$

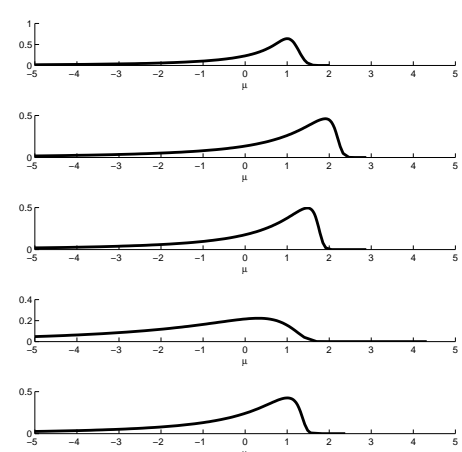

(b) $p\left(\mu_{1} \mid \mathcal{X}_{k, \text { post }}\right)$

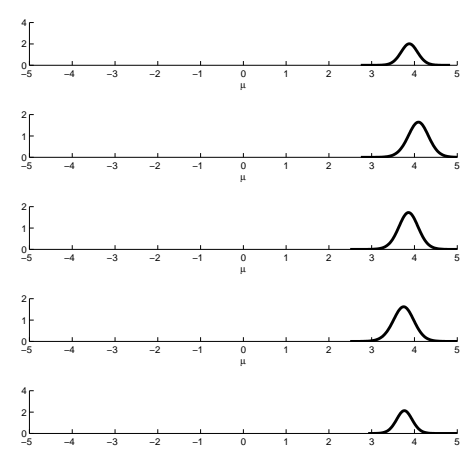

(e) $p\left(\mu_{1} \mid \mathcal{X}_{k, \text { pre }}\right)$

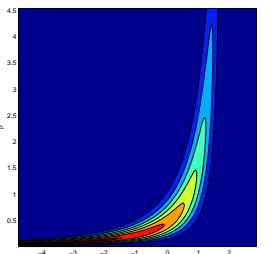

(g-3) $p\left(\mu, \beta \mid \mathcal{X}_{3, \text { post }}\right)$

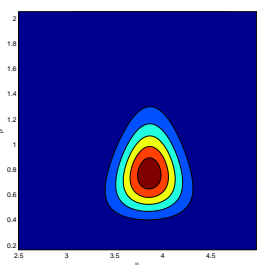

(h-3) $p\left(\mu, \beta \mid \mathcal{X}_{3, \text { pre }}\right)$

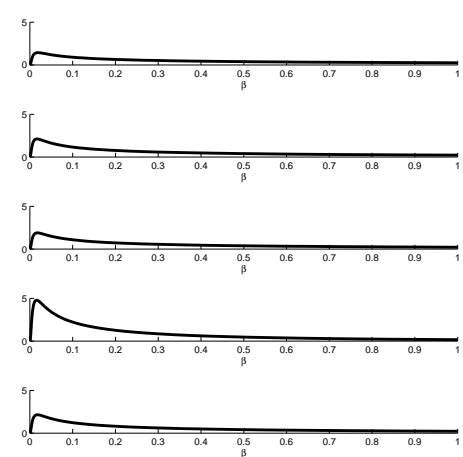

(c) $p\left(\beta_{1} \mid \mathcal{X}_{k, \text { post }}\right)$

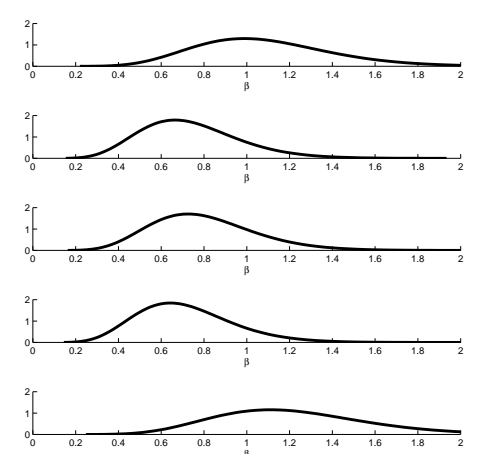

(f) $p\left(\beta_{1} \mid \mathcal{X}_{k, \text { pre }}\right)$

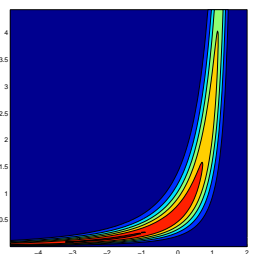

(g-1) $p\left(\mu, \beta \mid \mathcal{X}_{1, \text { post }}\right)$

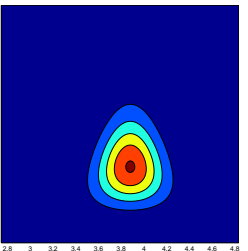

(h-1) $p\left(\mu, \beta \mid \mathcal{X}_{1, \text { pre }}\right)$

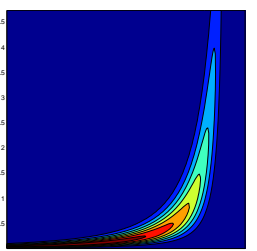

(g-2) $p\left(\mu, \beta \mid \mathcal{X}_{2, \text { post }}\right)$

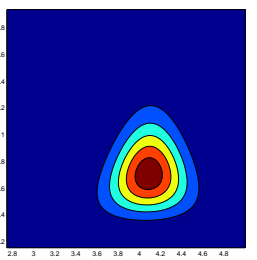

(h-2) $p\left(\mu, \beta \mid \mathcal{X}_{2, \text { pre }}\right)$

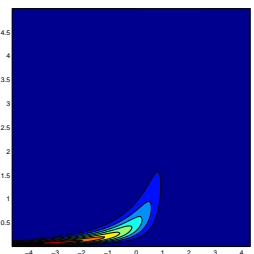

(g-4) $p\left(\mu, \beta \mid \mathcal{X}_{4, \text { post }}\right)$

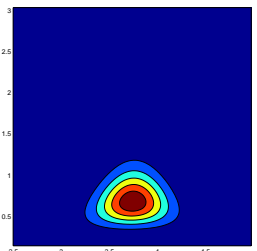

(h-4) $p\left(\mu, \beta \mid \mathcal{X}_{4, \text { pre }}\right)$

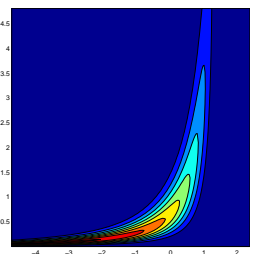

(g-5) $p\left(\mu, \beta \mid \mathcal{X}_{5, \text { post }}\right)$

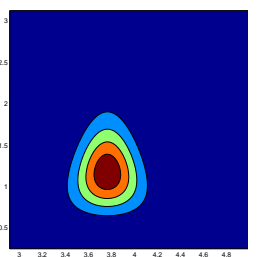

(h-5) $p\left(\mu, \beta \mid \mathcal{X}_{5, \text { pre }}\right)$

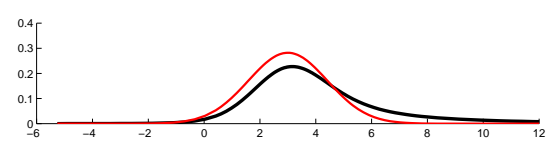

(i-1) $p\left(y \mid \mathcal{X}_{1, \text { pre }}, \mathcal{X}_{1, \text { post }}\right)$

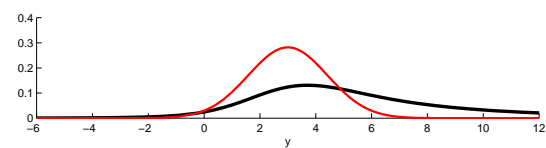

(i-4) $p\left(y \mid \mathcal{X}_{4, \text { pre }}, \mathcal{X}_{4, \text { post }}\right)$

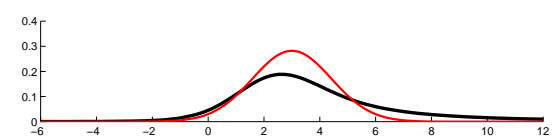

(i-2) $p\left(y \mid \mathcal{X}_{2, \text { pre }}, \mathcal{X}_{2, \text { post }}\right)$

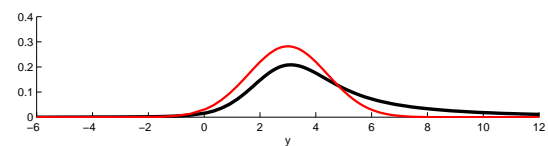

(i-5) $p\left(y \mid \mathcal{X}_{5, \text { pre }}, \mathcal{X}_{5, \text { post }}\right)$

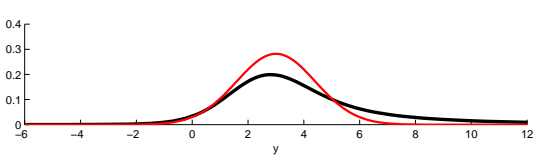

(i-3) $p\left(y \mid \mathcal{X}_{3, \text { pre }}, \mathcal{X}_{3, \text { post }}\right)$

Figure 14S: Bayesian inferences on five datasets, each of which contains $n_{v}=2$ detect data out of $n=24$ samples. 


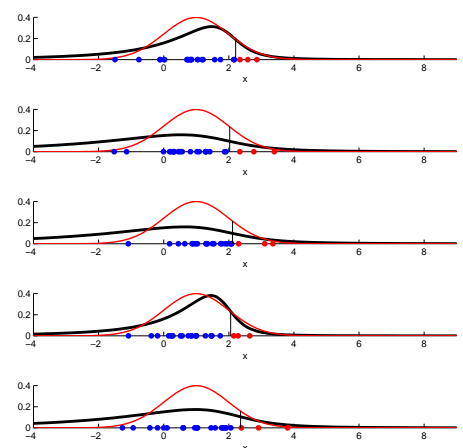

(a) $p\left(c_{\text {post }} \mid \mathcal{X}_{k, \text { post }}\right)$

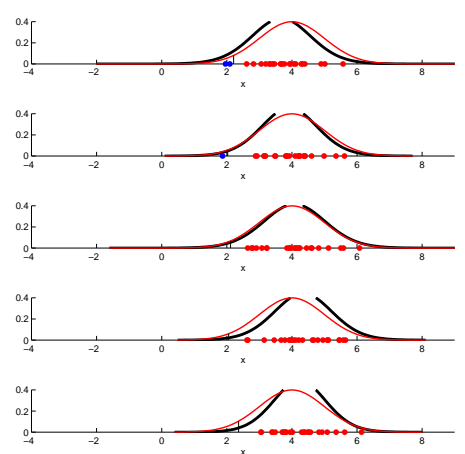

(d) $p\left(c_{\text {pre }} \mid \mathcal{X}_{k \text {,pre }}\right)$

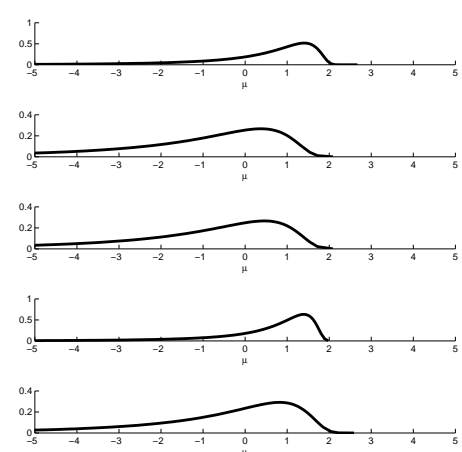

(b) $p\left(\mu_{1} \mid \mathcal{X}_{k, \text { post }}\right)$

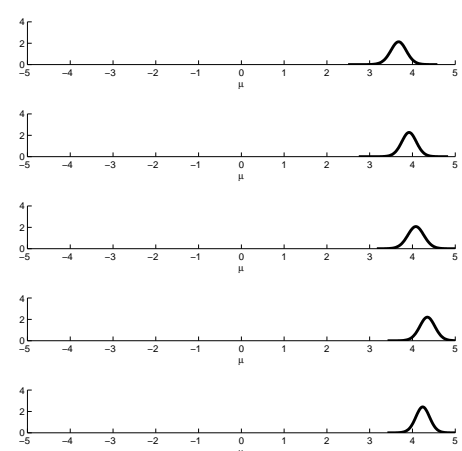

(e) $p\left(\mu_{1} \mid \mathcal{X}_{k, \text { pre }}\right)$

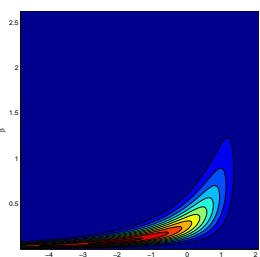

(g-3) $p\left(\mu, \beta \mid \mathcal{X}_{3, \text { post }}\right)$

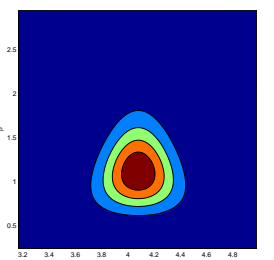

(h-3) $p\left(\mu, \beta \mid \mathcal{X}_{3, \text { pre }}\right)$

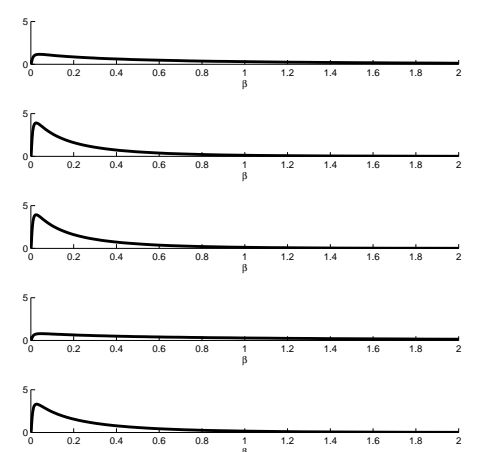

(c) $p\left(\beta_{1} \mid \mathcal{X}_{k, \text { post }}\right)$

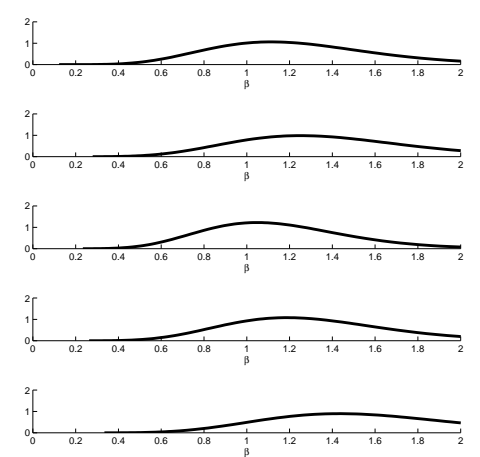

(f) $p\left(\beta_{1} \mid \mathcal{X}_{k, \text { pre }}\right)$

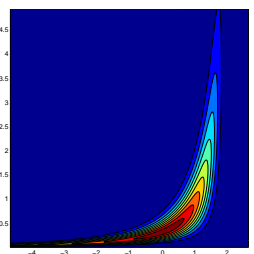

(g-1) $p\left(\mu, \beta \mid \mathcal{X}_{1, \text { post }}\right)$

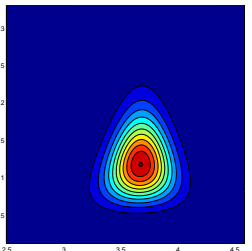

(h-1) $p\left(\mu, \beta \mid \mathcal{X}_{1, \text { pre }}\right)$

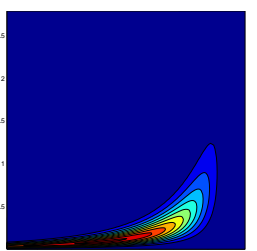

(g-2) $p\left(\mu, \beta \mid \mathcal{X}_{2, \text { post }}\right)$

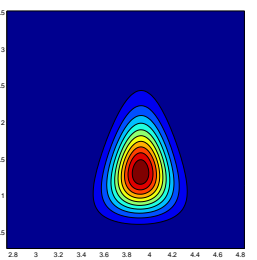

(h-2) $p\left(\mu, \beta \mid \mathcal{X}_{2, \text { pre }}\right)$

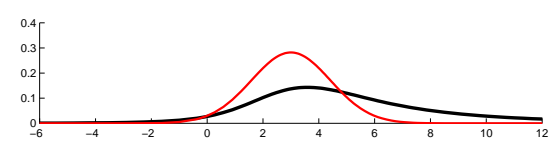

(i-2) $p\left(y \mid \mathcal{X}_{2, \text { pre }}, \mathcal{X}_{2, \text { post }}\right)$

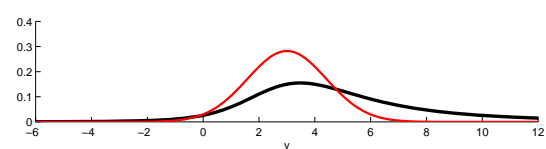

(i-5) $p\left(y \mid \mathcal{X}_{5, \text { pre }}, \mathcal{X}_{5, \text { post }}\right)$

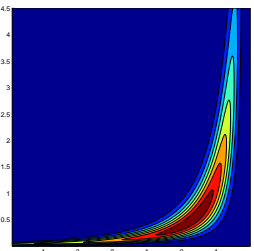

(g-4) $p\left(\mu, \beta \mid \mathcal{X}_{4, \text { post }}\right)$

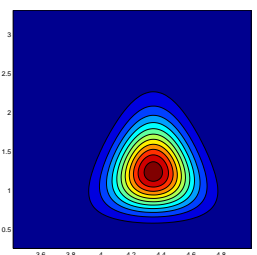

(h-4) $p\left(\mu, \beta \mid \mathcal{X}_{4, \text { pre }}\right)$

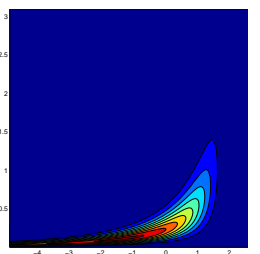

(g-5) $p\left(\mu, \beta \mid \mathcal{X}_{5, \text { post }}\right)$

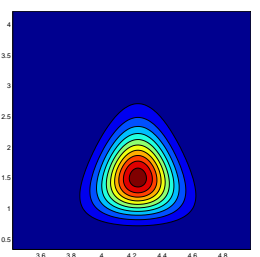

(h-5) $p\left(\mu, \beta \mid \mathcal{X}_{5, \text { pre }}\right)$

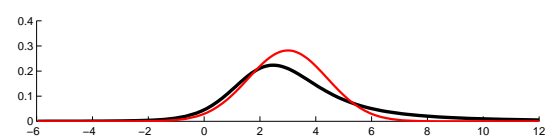

(i-1) $p\left(y \mid \mathcal{X}_{1, \text { pre }}, \mathcal{X}_{1, \text { post }}\right)$

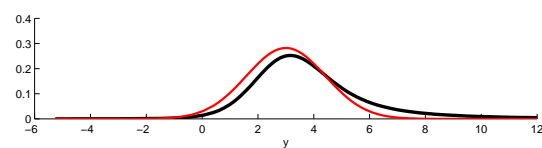

(i-4) $p\left(y \mid \mathcal{X}_{4, \text { pre }}, \mathcal{X}_{4, \text { post }}\right)$

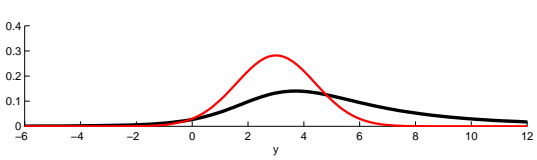

$(\mathrm{i}-3) p\left(y \mid \mathcal{X}_{3, \text { pre }}, \mathcal{X}_{3, \text { post }}\right)$

Figure 15S: Bayesian inferences on five datasets, each of which contains $n_{v}=3$ detect data out of $n=24$ samples. 


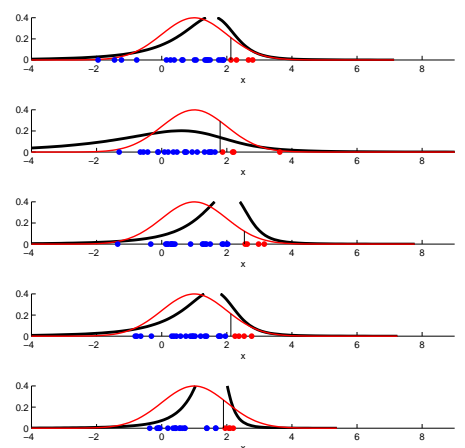

(a) $p\left(c_{\text {post }} \mid \mathcal{X}_{k, \text { post }}\right)$

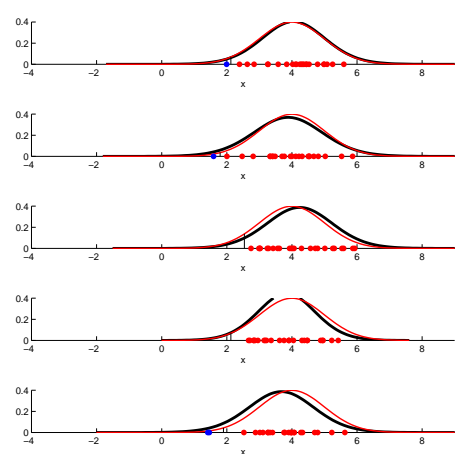

(d) $p\left(c_{\text {pre }} \mid \mathcal{X}_{k, \text { pre }}\right)$

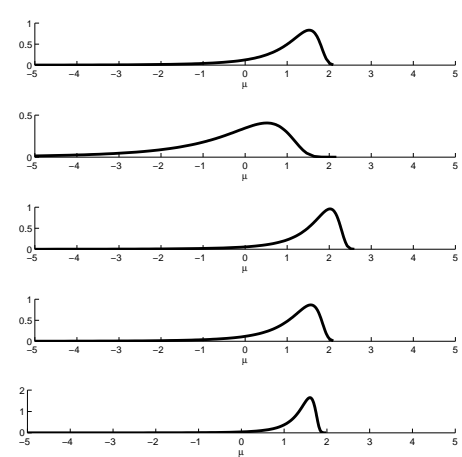

(b) $p\left(\mu_{1} \mid \mathcal{X}_{k, \text { post }}\right)$

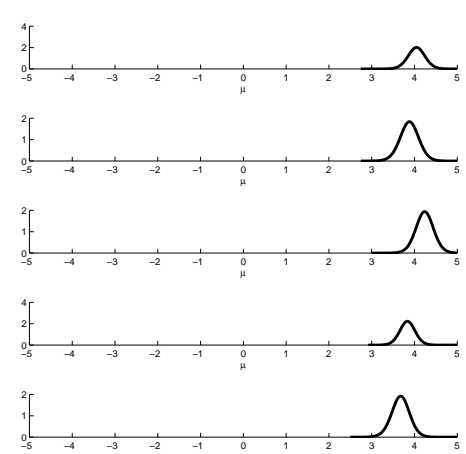

(e) $p\left(\mu_{1} \mid \mathcal{X}_{k, \text { pre }}\right)$

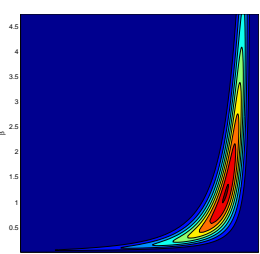

(g-3) $p\left(\mu, \beta \mid \mathcal{X}_{3, \text { post }}\right)$

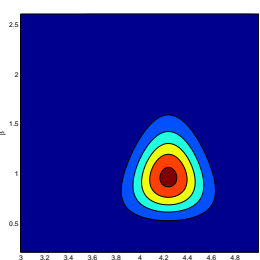

(h-3) $p\left(\mu, \beta \mid \mathcal{X}_{3, \text { pre }}\right)$

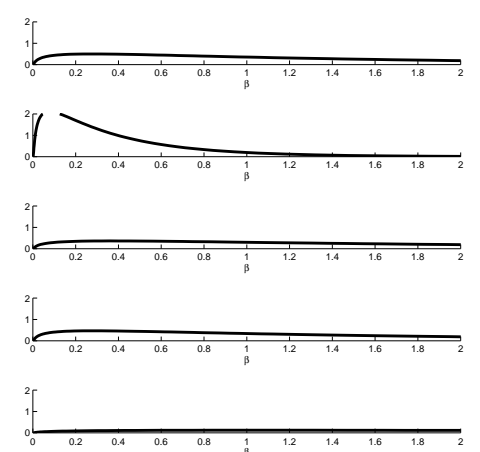

(c) $p\left(\beta_{1} \mid \mathcal{X}_{k, \text { post }}\right)$

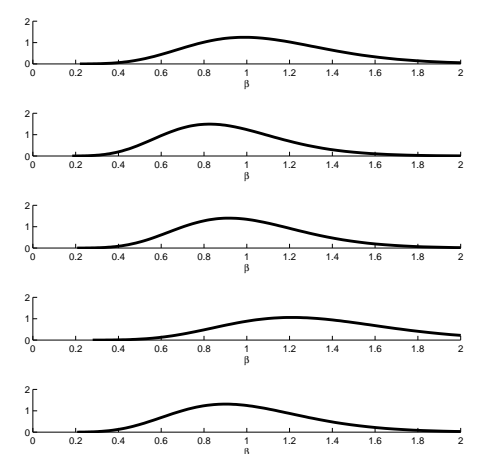

(f) $p\left(\beta_{1} \mid \mathcal{X}_{k, \text { pre }}\right)$

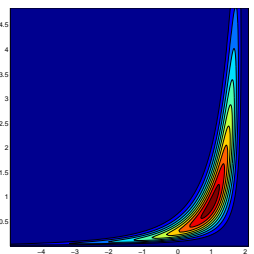

(g-1) $p\left(\mu, \beta \mid \mathcal{X}_{1, \text { post }}\right)$

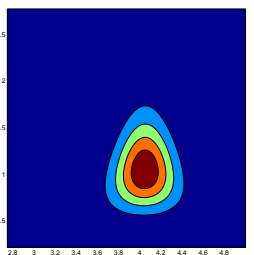

(h-1) $p\left(\mu, \beta \mid \mathcal{X}_{1, \text { pre }}\right)$

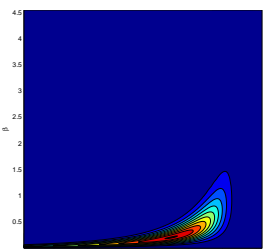

(g-2) $p\left(\mu, \beta \mid \mathcal{X}_{2, \text { post }}\right)$

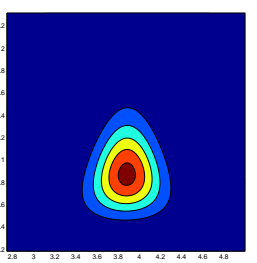

(h-2) $p\left(\mu, \beta \mid \mathcal{X}_{2, \text { pre }}\right)$

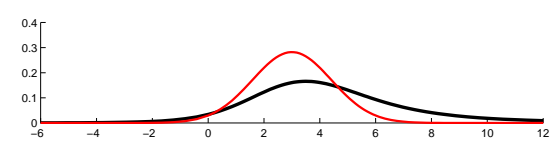

(i-2) $p\left(y \mid \mathcal{X}_{2, \text { pre }}, \mathcal{X}_{2, \text { post }}\right)$

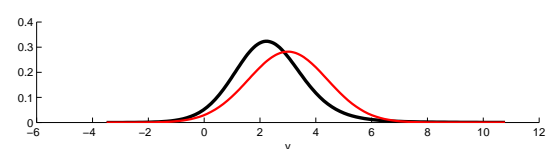

(i-5) $p\left(y \mid \mathcal{X}_{5, \text { pre }}, \mathcal{X}_{5, \text { post }}\right)$
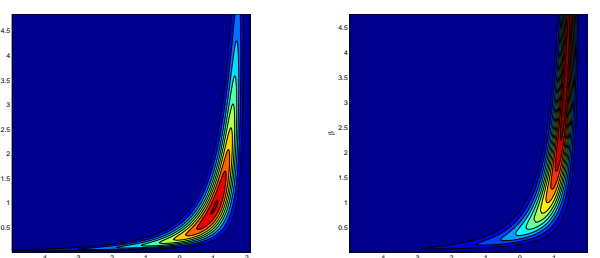

(g-4) $p\left(\mu, \beta \mid \mathcal{X}_{4, \text { post }}\right)$

$(\mathrm{g}-5) p\left(\mu, \beta \mid \mathcal{X}_{5, \text { post }}\right)$
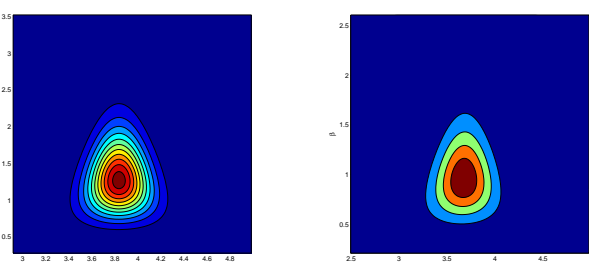

(h-4) $p\left(\mu, \beta \mid \mathcal{X}_{4, \text { pre }}\right)$

(h-5) $p\left(\mu, \beta \mid \mathcal{X}_{5, \text { pre }}\right)$

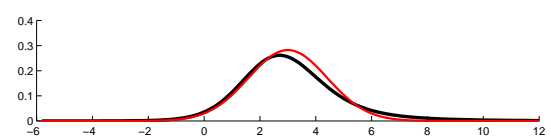

(i-1) $p\left(y \mid \mathcal{X}_{1, \text { pre }}, \mathcal{X}_{1, \text { post }}\right)$

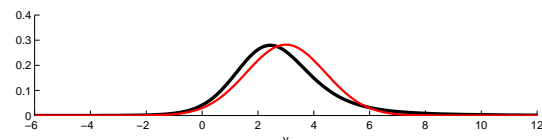

(i-4) $p\left(y \mid \mathcal{X}_{4, \text { pre }}, \mathcal{X}_{4, \text { post }}\right)$

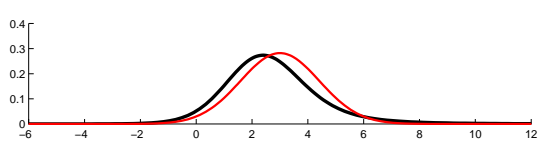

(i-3) $p\left(y \mid \mathcal{X}_{3, \text { pre }}, \mathcal{X}_{3, \text { post }}\right)$

Figure 16S: Bayesian inferences on five datasets, each of which contains $n_{v}=4$ detect data out of $n=24$ samples. 


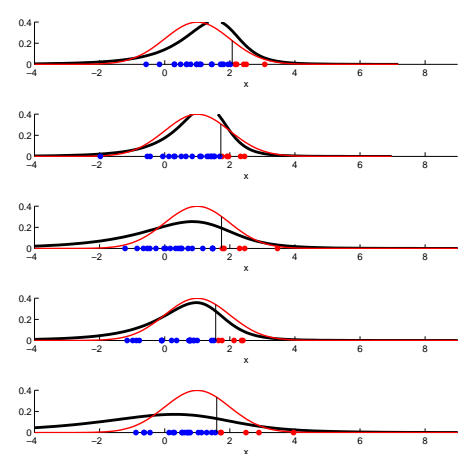

(a) $p\left(c_{\text {post }} \mid \mathcal{X}_{k, \text { post }}\right)$

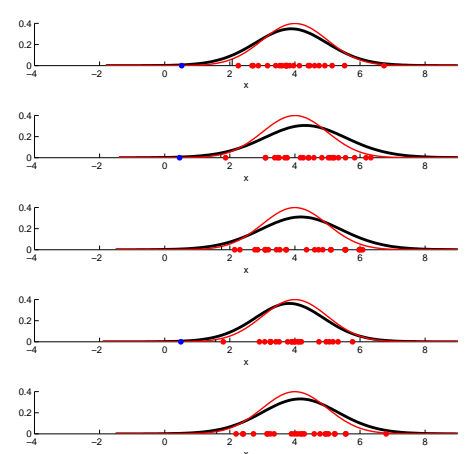

(d) $p\left(c_{\text {pre }} \mid \mathcal{X}_{k, \text { pre }}\right)$

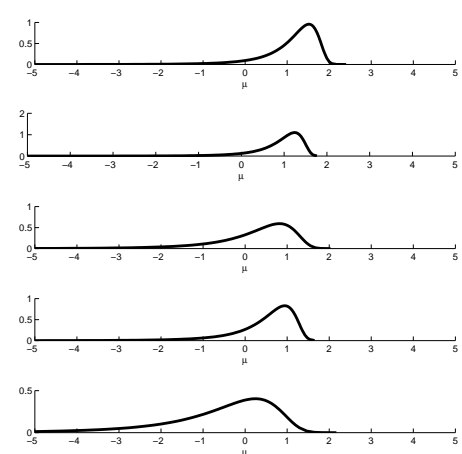

(b) $p\left(\mu_{1} \mid \mathcal{X}_{k, \text { post }}\right)$

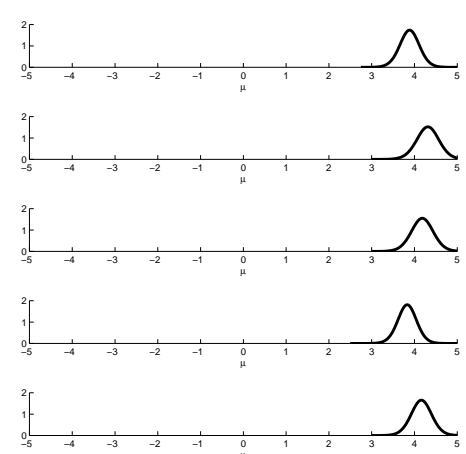

(e) $p\left(\mu_{1} \mid \mathcal{X}_{k, \text { pre }}\right)$

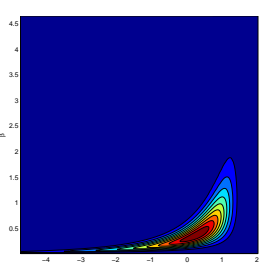

(g-3) $p\left(\mu, \beta \mid \mathcal{X}_{3, \text { post }}\right)$

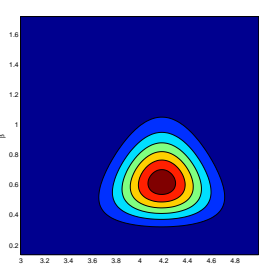

(h-3) $p\left(\mu, \beta \mid \mathcal{X}_{3, \text { pre }}\right)$

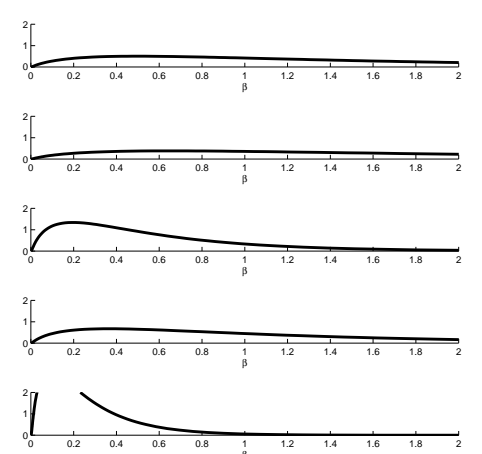

(c) $p\left(\beta_{1} \mid \mathcal{X}_{k, \text { post }}\right)$

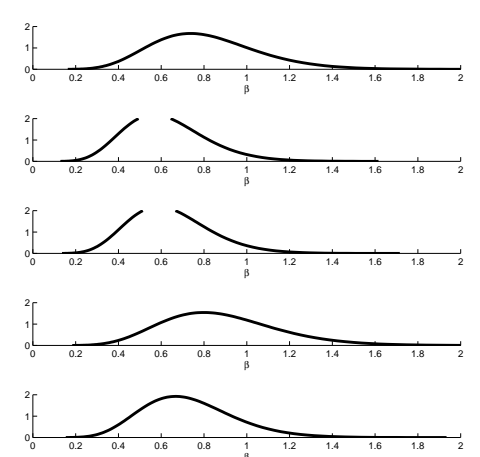

(f) $p\left(\beta_{1} \mid \mathcal{X}_{k, \text { pre }}\right)$

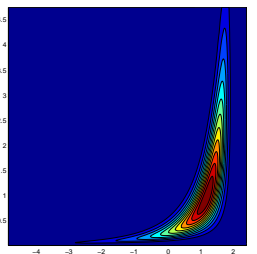

(g-1) $p\left(\mu, \beta \mid \mathcal{X}_{1, \text { post }}\right)$

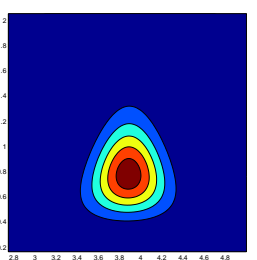

(h-1) $p\left(\mu, \beta \mid \mathcal{X}_{1, \text { pre }}\right)$

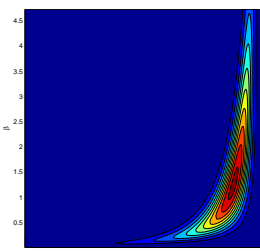

(g-2) $p\left(\mu, \beta \mid \mathcal{X}_{2, \text { post }}\right)$

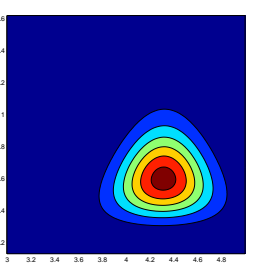

(h-2) $p\left(\mu, \beta \mid \mathcal{X}_{2, \text { pre }}\right)$

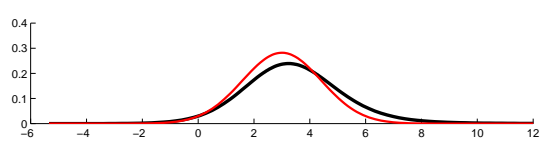

(i-2) $p\left(y \mid \mathcal{X}_{2, \text { pre }}, \mathcal{X}_{2, \text { post }}\right)$

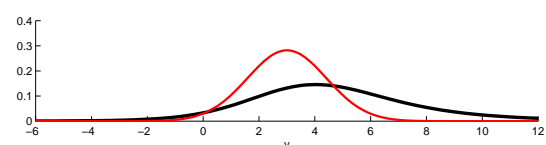

(i-5) $p\left(y \mid \mathcal{X}_{5, \text { pre }}, \mathcal{X}_{5, \text { post }}\right)$
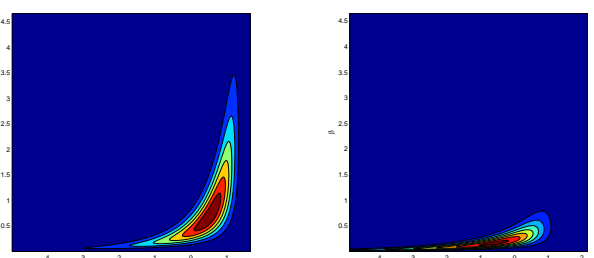

(g-4) $p\left(\mu, \beta \mid \mathcal{X}_{4, \text { post }}\right)$

(g-5) $p\left(\mu, \beta \mid \mathcal{X}_{5, \text { post }}\right)$
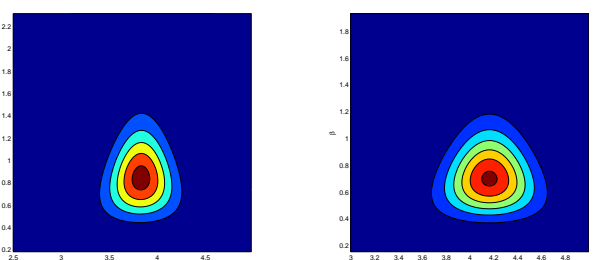

(h-4) $p\left(\mu, \beta \mid \mathcal{X}_{4, \text { pre }}\right)$

(h-5) $p\left(\mu, \beta \mid \mathcal{X}_{5, \text { pre }}\right)$

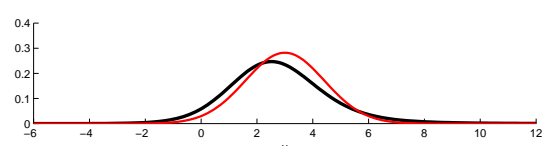

(i-1) $p\left(y \mid \mathcal{X}_{1, \text { pre }}, \mathcal{X}_{1, \text { post }}\right)$

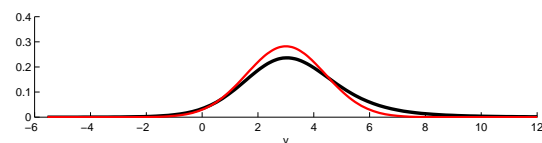

(i-4) $p\left(y \mid \mathcal{X}_{4, \text { pre }}, \mathcal{X}_{4, \text { post }}\right)$

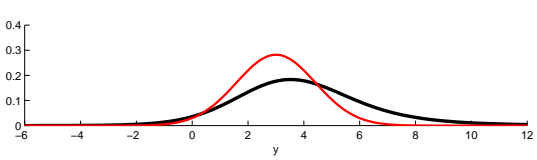

(i-3) $p\left(y \mid \mathcal{X}_{3, \text { pre }}, \mathcal{X}_{3, \text { post }}\right)$

Figure 17S: Bayesian inferences on five datasets, each of which contains $n_{v}=5$ detect data out of $n=24$ samples. 


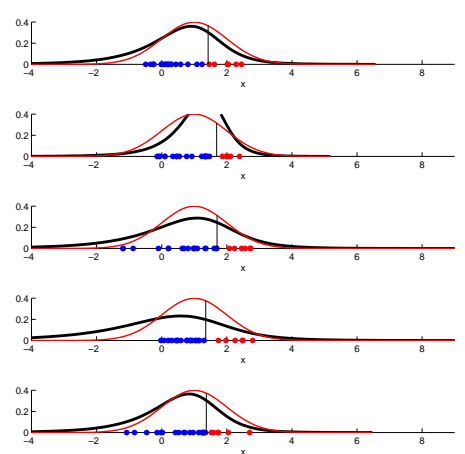

(a) $p\left(c_{\text {post }} \mid \mathcal{X}_{k, \text { post }}\right)$

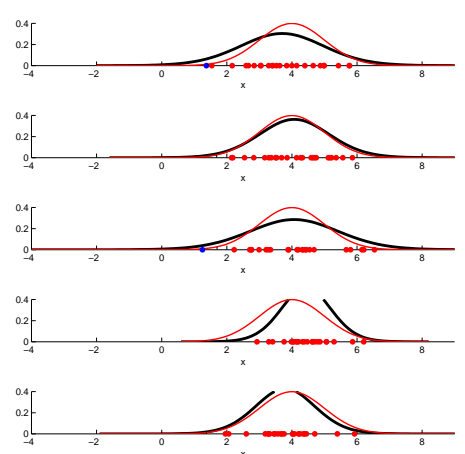

(d) $p\left(c_{\text {pre }} \mid \mathcal{X}_{k, \text { pre }}\right)$

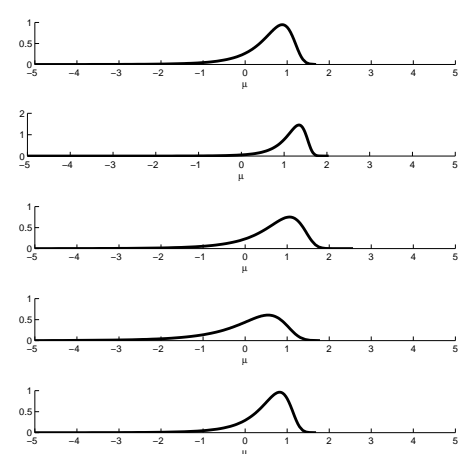

(b) $p\left(\mu_{1} \mid \mathcal{X}_{k, \text { post }}\right)$

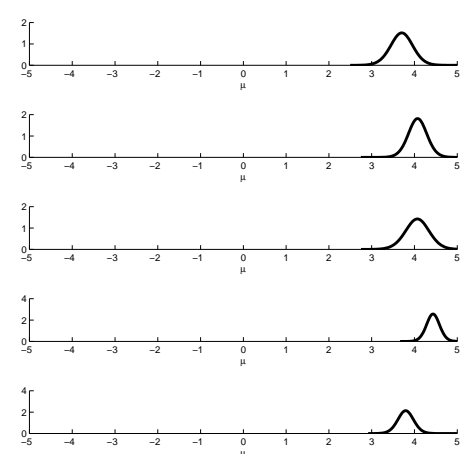

(e) $p\left(\mu_{1} \mid \mathcal{X}_{k, \text { pre }}\right)$

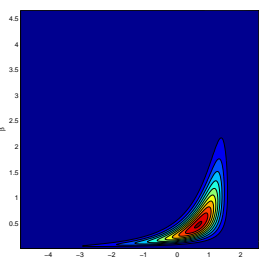

(g-3) $p\left(\mu, \beta \mid \mathcal{X}_{3, \text { post }}\right)$

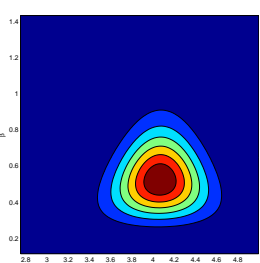

(h-3) $p\left(\mu, \beta \mid \mathcal{X}_{3, \text { pre }}\right)$

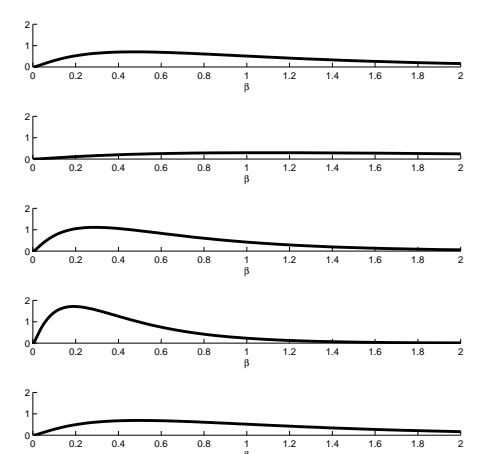

(c) $p\left(\beta_{1} \mid \mathcal{X}_{k, \text { post }}\right)$

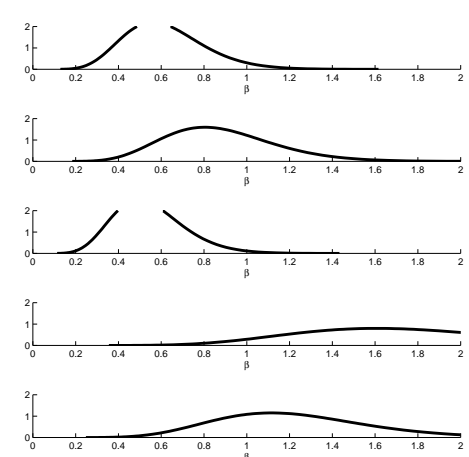

(f) $p\left(\beta_{1} \mid \mathcal{X}_{k, \text { pre }}\right)$

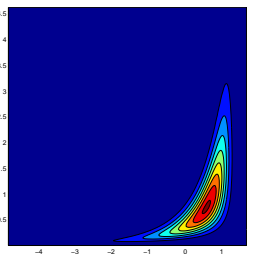

(g-1) $p\left(\mu, \beta \mid \mathcal{X}_{1, \text { post }}\right)$

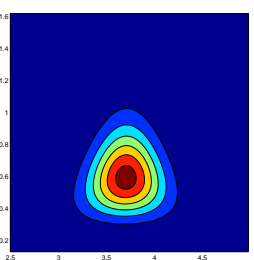

(h-1) $p\left(\mu, \beta \mid \mathcal{X}_{1, \text { pre }}\right)$

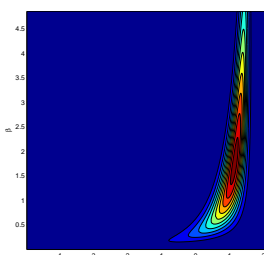

(g-2) $p\left(\mu, \beta \mid \mathcal{X}_{2, \text { post }}\right)$

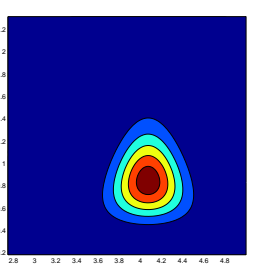

(h-2) $p\left(\mu, \beta \mid \mathcal{X}_{2, \text { pre }}\right)$

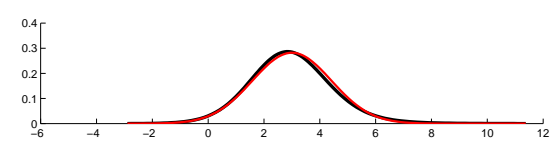

(i-2) $p\left(y \mid \mathcal{X}_{2, \text { pre }}, \mathcal{X}_{2, \text { post }}\right)$

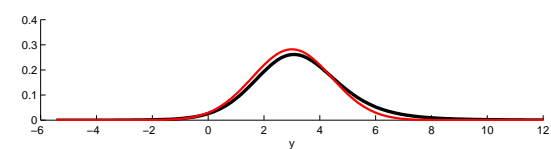

(i-5) $p\left(y \mid \mathcal{X}_{5, \text { pre }}, \mathcal{X}_{5, \text { post }}\right)$
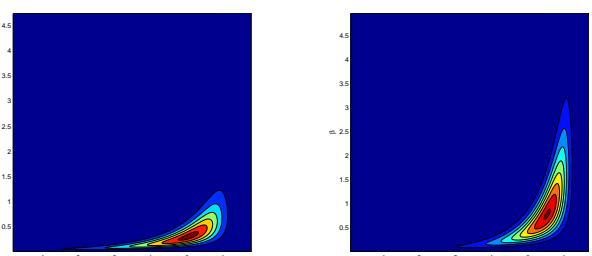

(g-4) $p\left(\mu, \beta \mid \mathcal{X}_{4, \text { post }}\right)$

$(\mathrm{g}-5) p\left(\mu, \beta \mid \mathcal{X}_{5, \text { post }}\right)$
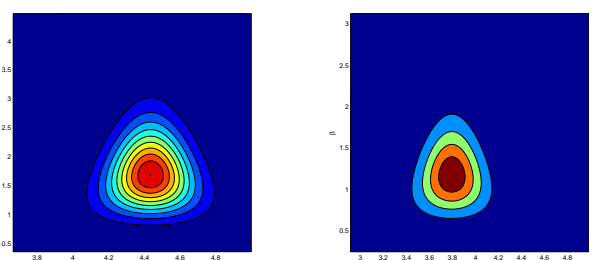

(h-4) $p\left(\mu, \beta \mid \mathcal{X}_{4, \text { pre }}\right) \quad(\mathrm{h}-5) p\left(\mu, \beta \mid \mathcal{X}_{5, \text { pre }}\right)$

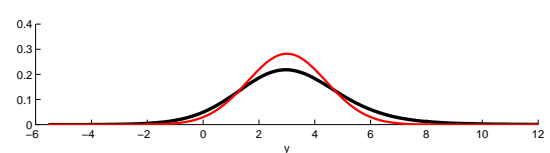

(i-1) $p\left(y \mid \mathcal{X}_{1, \text { pre }}, \mathcal{X}_{1, \text { post }}\right)$

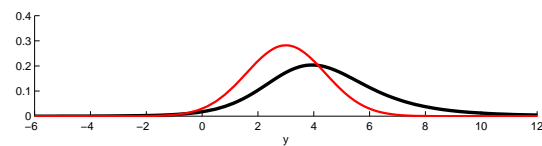

(i-4) $p\left(y \mid \mathcal{X}_{4, \text { pre }}, \mathcal{X}_{4, \text { post }}\right)$

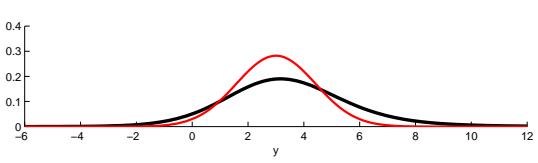

(i-3) $p\left(y \mid \mathcal{X}_{3, \text { pre }}, \mathcal{X}_{3, \text { post }}\right)$

Figure 18S: Bayesian inferences on five datasets, each of which contains $n_{v}=6$ detect data out of $n=24$ samples. 


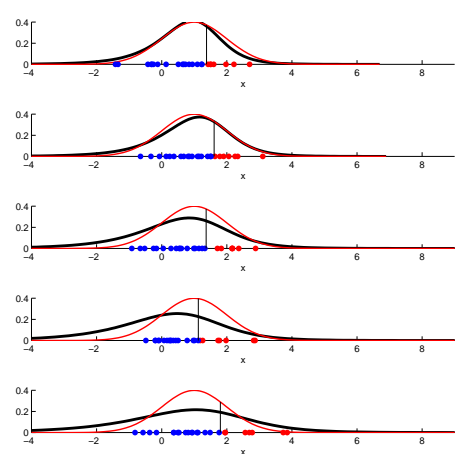

(a) $p\left(c_{\text {post }} \mid \mathcal{X}_{k \text {,post }}\right)$

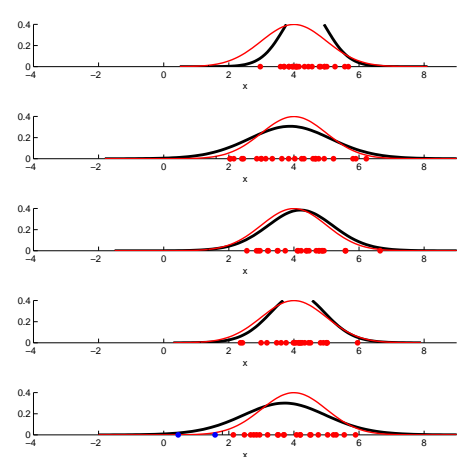

(d) $p\left(c_{\text {pre }} \mid \mathcal{X}_{k, \text { pre }}\right)$

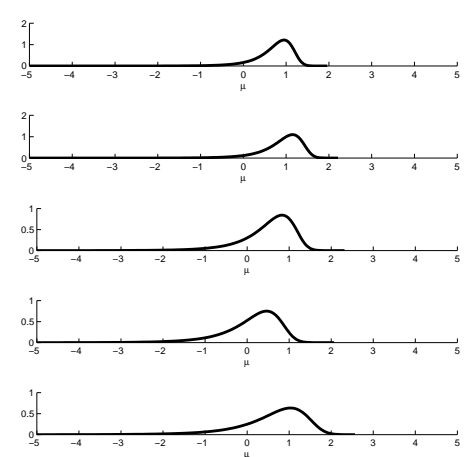

(b) $p\left(\mu_{1} \mid \mathcal{X}_{k, \text { post }}\right)$

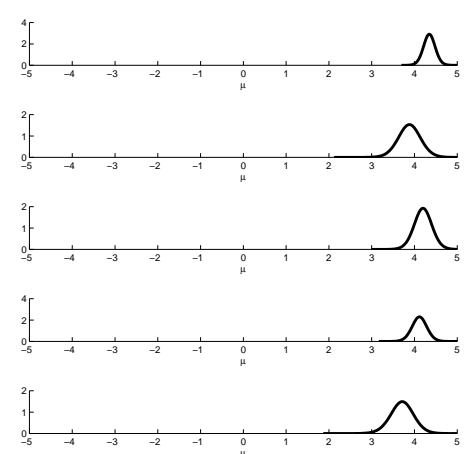

(e) $p\left(\mu_{1} \mid \mathcal{X}_{k, \text { pre }}\right)$

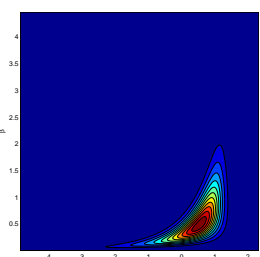

(g-3) $p\left(\mu, \beta \mid \mathcal{X}_{3, \text { post }}\right)$

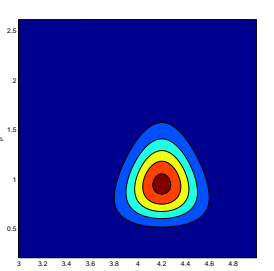

(h-3) $p\left(\mu, \beta \mid \mathcal{X}_{3, \text { pre }}\right)$

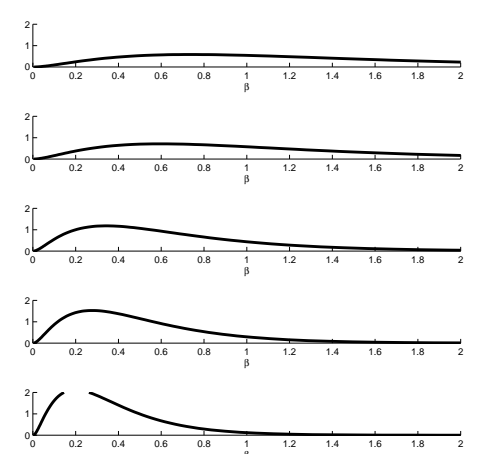

(c) $p\left(\beta_{1} \mid \mathcal{X}_{k, \text { post }}\right)$

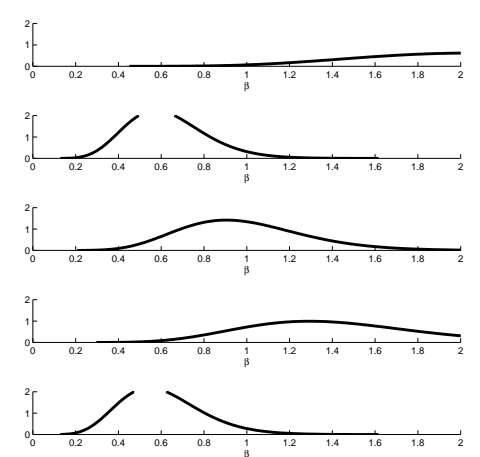

(f) $p\left(\beta_{1} \mid \mathcal{X}_{k, \text { pre }}\right)$

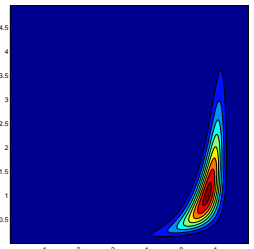

(g-1) $p\left(\mu, \beta \mid \mathcal{X}_{1, \text { post }}\right)$

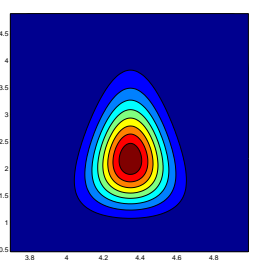

(h-1) $p\left(\mu, \beta \mid \mathcal{X}_{1, \text { pre }}\right)$

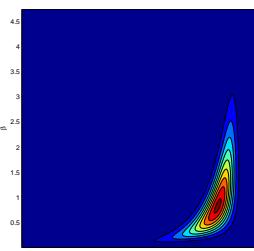

$(\mathrm{g}-2) p\left(\mu, \beta \mid \mathcal{X}_{2, \text { post }}\right)$

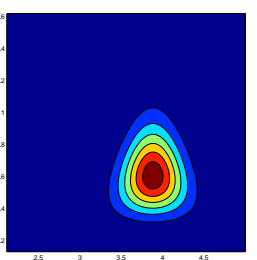

(h-2) $p\left(\mu, \beta \mid \mathcal{X}_{2, \text { pre }}\right)$

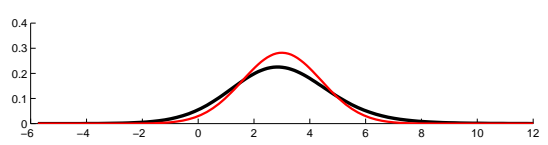

(i-2) $p\left(y \mid \mathcal{X}_{2, \text { pre }}, \mathcal{X}_{2, \text { post }}\right)$

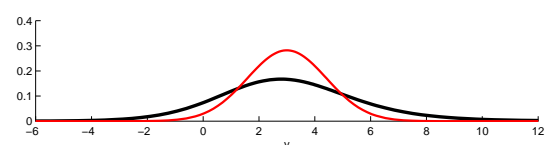

(i-5) $p\left(y \mid \mathcal{X}_{5, \text { pre }}, \mathcal{X}_{5, \text { post }}\right)$
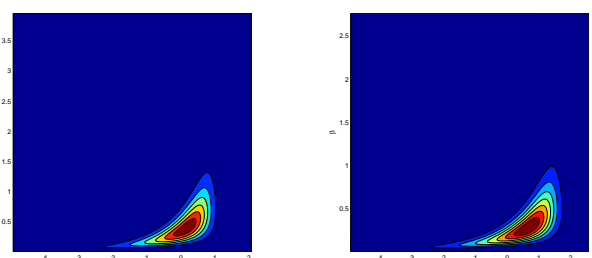

(g-4) $p\left(\mu, \beta \mid \mathcal{X}_{4, \text { post }}\right)$

$(\mathrm{g}-5) p\left(\mu, \beta \mid \mathcal{X}_{5, \text { post }}\right)$
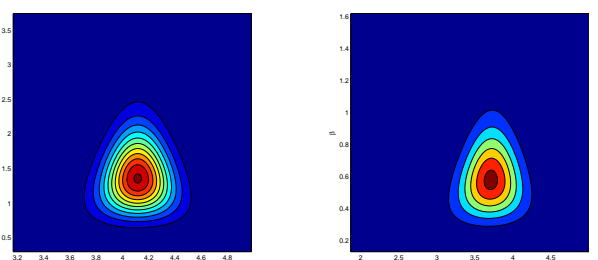

(h-4) $p\left(\mu, \beta \mid \mathcal{X}_{4, \text { pre }}\right)$

(h-5) $p\left(\mu, \beta \mid \mathcal{X}_{5, \text { pre }}\right)$

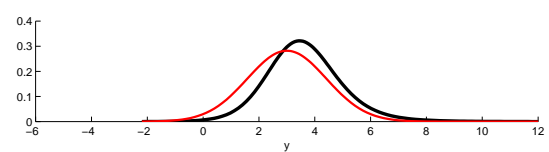

(i-1) $p\left(y \mid \mathcal{X}_{1, \text { pre }}, \mathcal{X}_{1, \text { post }}\right)$

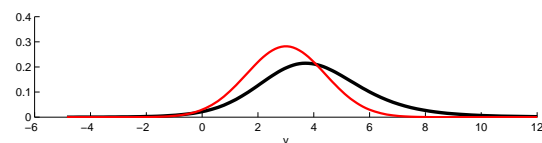

(i-4) $p\left(y \mid \mathcal{X}_{4, \text { pre }}, \mathcal{X}_{4, \text { post }}\right)$

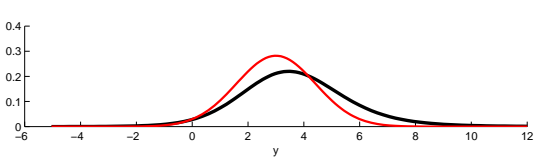

(i-3) $p\left(y \mid \mathcal{X}_{3, \text { pre }}, \mathcal{X}_{3, \text { post }}\right)$

Figure 19S: Bayesian inferences on five datasets, each of which contains $n_{v}=7$ detect data out of $n=24$ samples. 


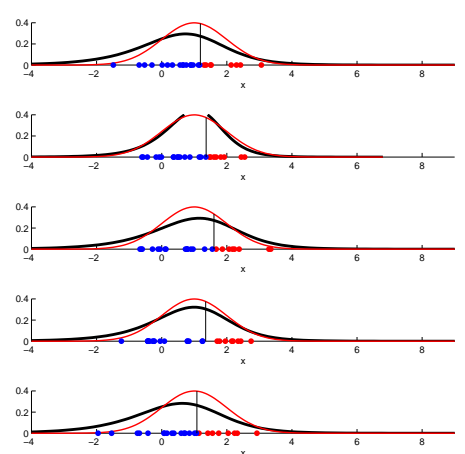

(a) $p\left(c_{\text {post }} \mid \mathcal{X}_{k, \text { post }}\right)$

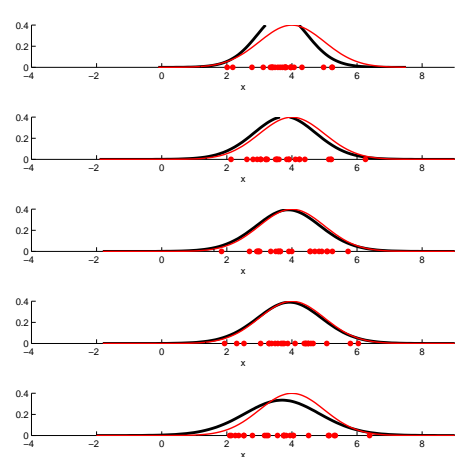

(d) $p\left(c_{\text {pre }} \mid \mathcal{X}_{k, \text { pre }}\right)$

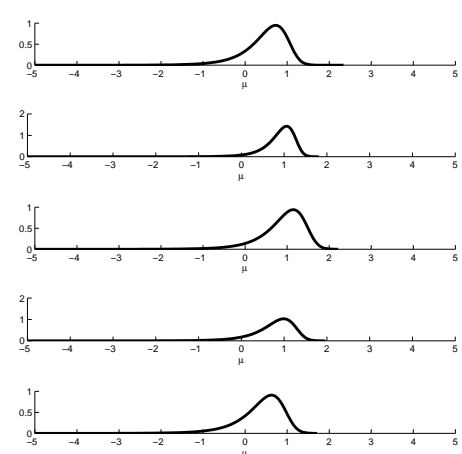

(b) $p\left(\mu_{1} \mid \mathcal{X}_{k, \text { post }}\right)$

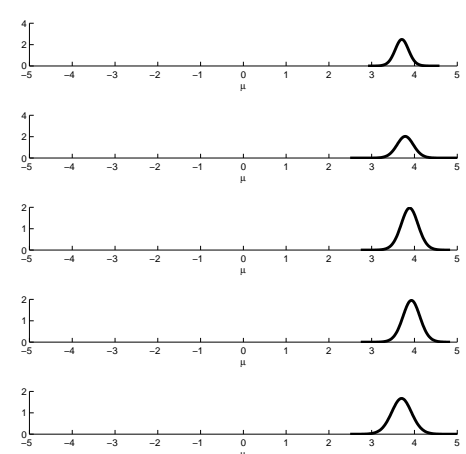

(e) $p\left(\mu_{1} \mid \mathcal{X}_{k, \text { pre }}\right)$

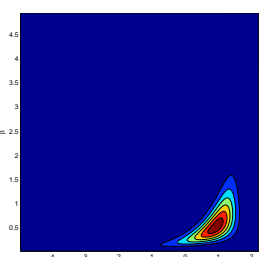

(g-3) $p\left(\mu, \beta \mid \mathcal{X}_{3, \text { post }}\right)$

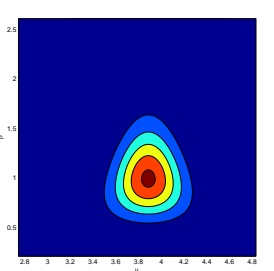

(h-3) $p\left(\mu, \beta \mid \mathcal{X}_{3, \text { pre }}\right)$

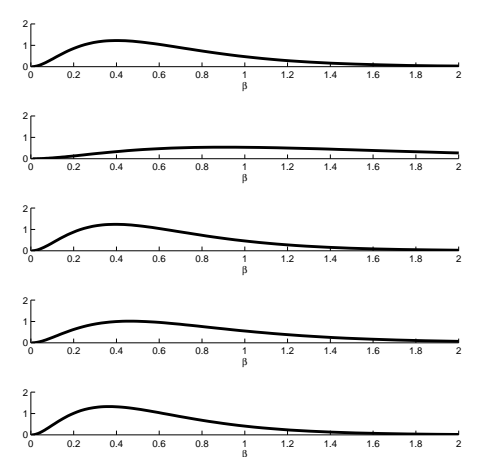

(c) $p\left(\beta_{1} \mid \mathcal{X}_{k, \text { post }}\right)$

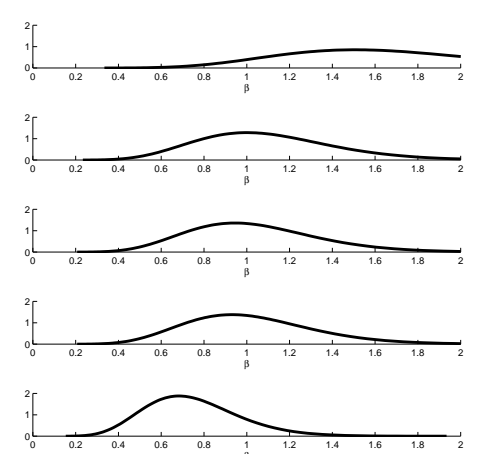

(f) $p\left(\beta_{1} \mid \mathcal{X}_{k, \text { pre }}\right)$

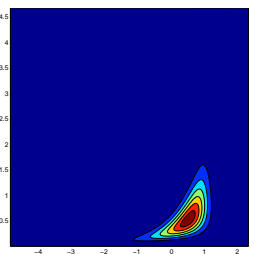

(g-1) $p\left(\mu, \beta \mid \mathcal{X}_{1, \text { post }}\right)$

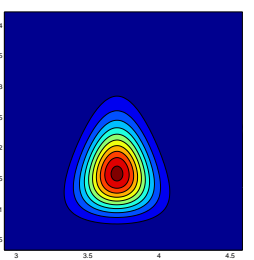

(h-1) $p\left(\mu, \beta \mid \mathcal{X}_{1, \text { pre }}\right)$

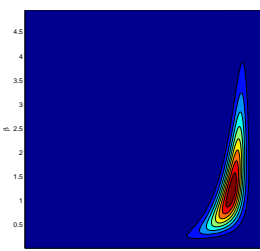

$(\mathrm{g}-2) p\left(\mu, \beta \mid \mathcal{X}_{2, \text { post }}\right)$

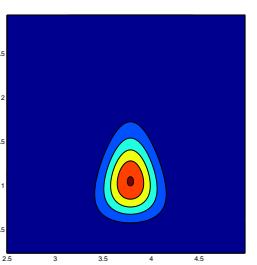

(h-2) $p\left(\mu, \beta \mid \mathcal{X}_{2, \text { pre }}\right)$

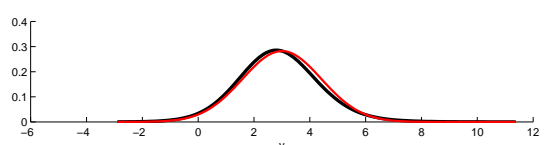

(i-2) $p\left(y \mid \mathcal{X}_{2, \text { pre }}, \mathcal{X}_{2, \text { post }}\right)$

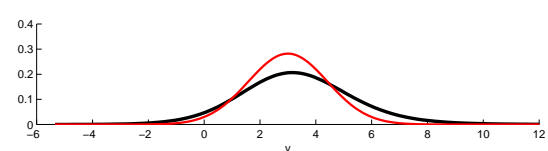

(i-5) $p\left(y \mid \mathcal{X}_{5, \text { pre }}, \mathcal{X}_{5, \text { post }}\right)$
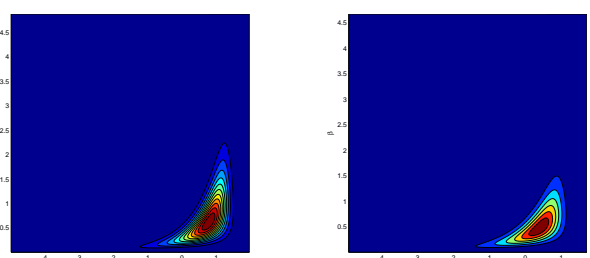

(g-4) $p\left(\mu, \beta \mid \mathcal{X}_{4, \text { post }}\right)$

(g-5) $p\left(\mu, \beta \mid \mathcal{X}_{5, \text { post }}\right)$
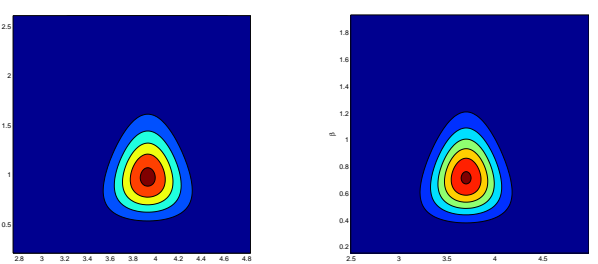

(h-4) $p\left(\mu, \beta \mid \mathcal{X}_{4, \text { pre }}\right)$

(h-5) $p\left(\mu, \beta \mid \mathcal{X}_{5, \text { pre }}\right)$

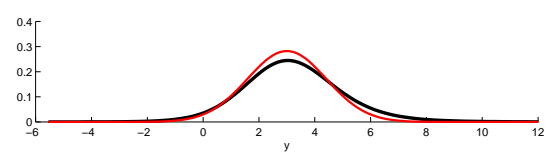

(i-1) $p\left(y \mid \mathcal{X}_{1, \text { pre }}, \mathcal{X}_{1, \text { post }}\right)$

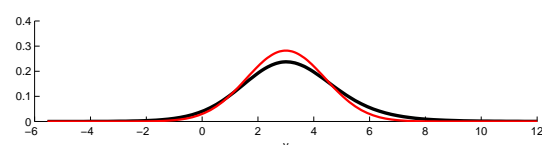

(i-4) $p\left(y \mid \mathcal{X}_{4, \text { pre }}, \mathcal{X}_{4, \text { post }}\right)$

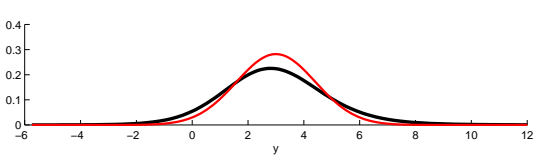

(i-3) $p\left(y \mid \mathcal{X}_{3, \text { pre }}, \mathcal{X}_{3, \text { post }}\right)$

Figure 20S: Bayesian inferences on five datasets, each of which contains $n_{v}=8$ detect data out of $n=24$ samples. 


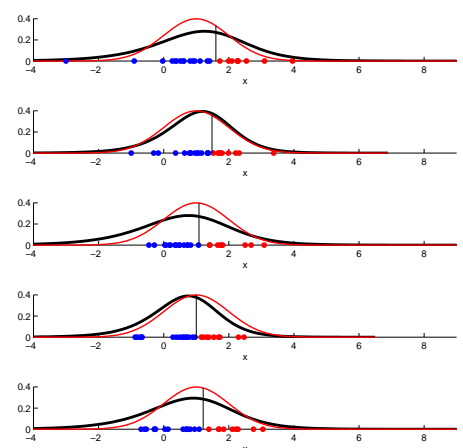

(a) $p\left(c_{\text {post }} \mid \mathcal{X}_{k, \text { post }}\right)$

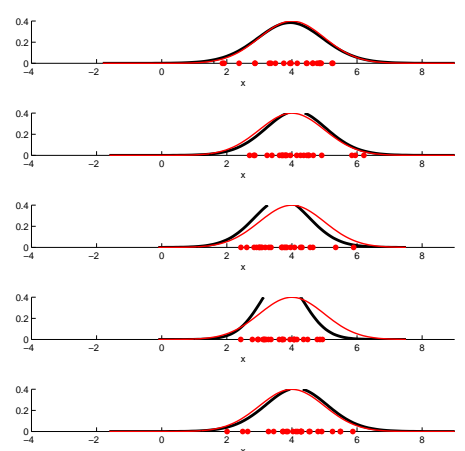

(d) $p\left(c_{\text {pre }} \mid \mathcal{X}_{k, \text { pre }}\right)$

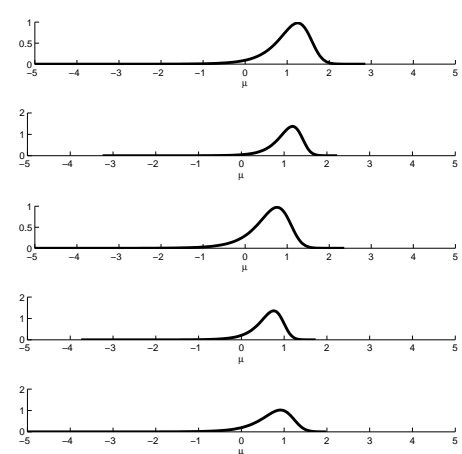

(b) $p\left(\mu_{1} \mid \mathcal{X}_{k, \text { post }}\right)$

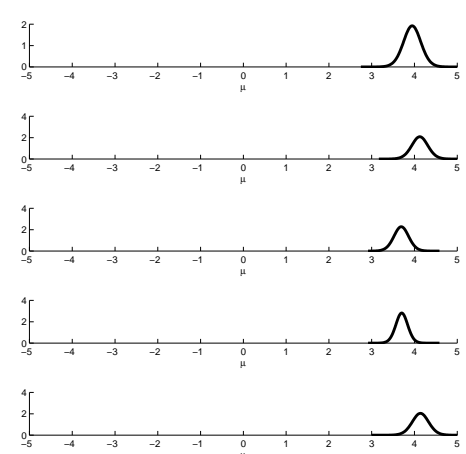

(e) $p\left(\mu_{1} \mid \mathcal{X}_{k, \text { pre }}\right)$

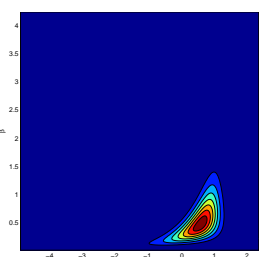

$(\mathrm{g}-3) p\left(\mu, \beta \mid \mathcal{X}_{3, \text { post }}\right)$

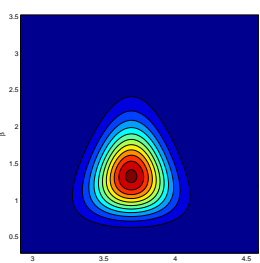

(h-3) $p\left(\mu, \beta \mid \mathcal{X}_{3, \text { pre }}\right)$

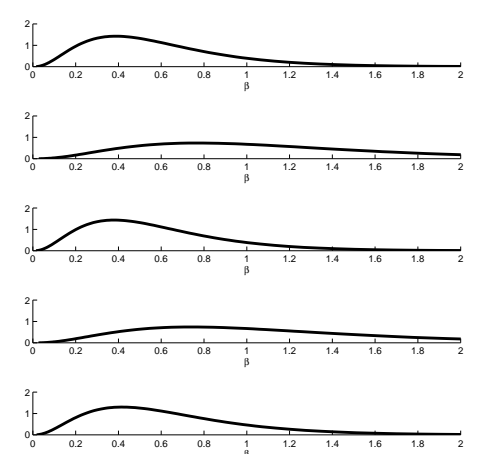

(c) $p\left(\beta_{1} \mid \mathcal{X}_{k, \text { post }}\right)$

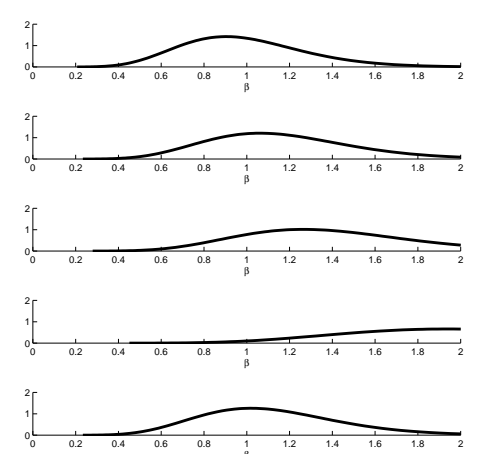

(f) $p\left(\beta_{1} \mid \mathcal{X}_{k, \text { pre }}\right)$

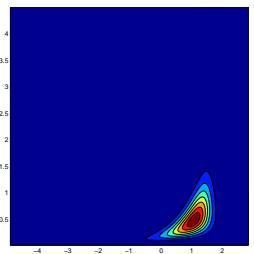

(g-1) $p\left(\mu, \beta \mid \mathcal{X}_{1, \text { post }}\right)$

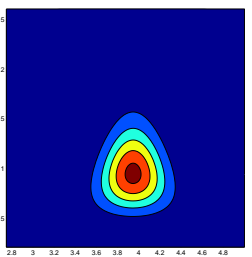

(h-1) $p\left(\mu, \beta \mid \mathcal{X}_{1, \text { pre }}\right)$

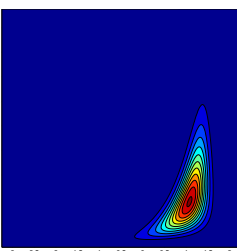

(g-2) $p\left(\mu, \beta \mid \mathcal{X}_{2, \text { post }}\right)$

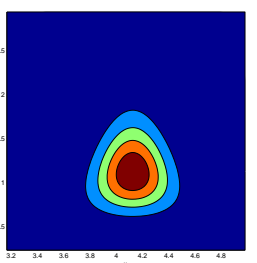

(h-2) $p\left(\mu, \beta \mid \mathcal{X}_{2, \text { pre }}\right)$

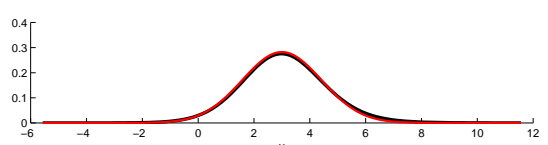

(i-2) $p\left(y \mid \mathcal{X}_{2, \text { pre }}, \mathcal{X}_{2, \text { post }}\right)$

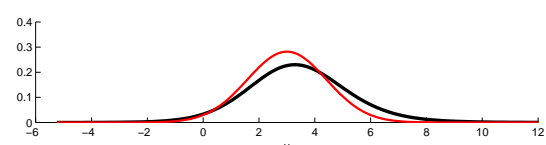

(i-5) $p\left(y \mid \mathcal{X}_{5, \text { pre }}, \mathcal{X}_{5, \text { post }}\right)$
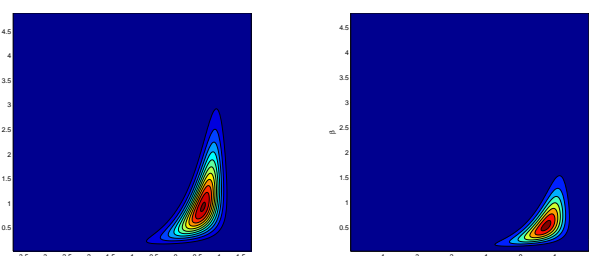

(g-4) $p\left(\mu, \beta \mid \mathcal{X}_{4, \text { post }}\right)$

(g-5) $p\left(\mu, \beta \mid \mathcal{X}_{5, \text { post }}\right)$
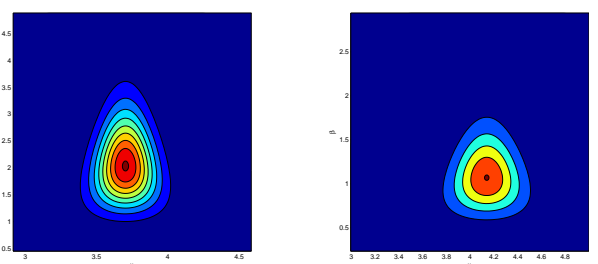

(h-4) $p\left(\mu, \beta \mid \mathcal{X}_{4, \text { pre }}\right)$

(h-5) $p\left(\mu, \beta \mid \mathcal{X}_{5, \text { pre }}\right)$

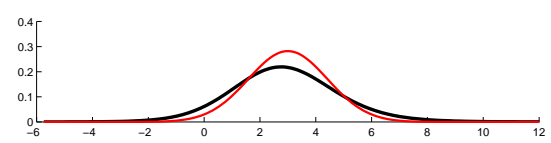

(i-1) $p\left(y \mid \mathcal{X}_{1, \text { pre }}, \mathcal{X}_{1, \text { post }}\right)$

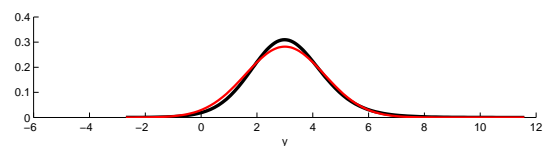

(i-4) $p\left(y \mid \mathcal{X}_{4, \text { pre }}, \mathcal{X}_{4, \text { post }}\right)$

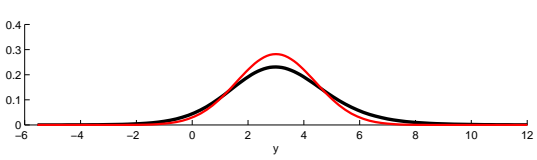

$(\mathrm{i}-3) p\left(y \mid \mathcal{X}_{3, \text { pre }}, \mathcal{X}_{3, \text { post }}\right)$

Figure 21S: Bayesian inferences on five datasets, each of which contains $n_{v}=9$ detect data out of $n=24$ samples. 


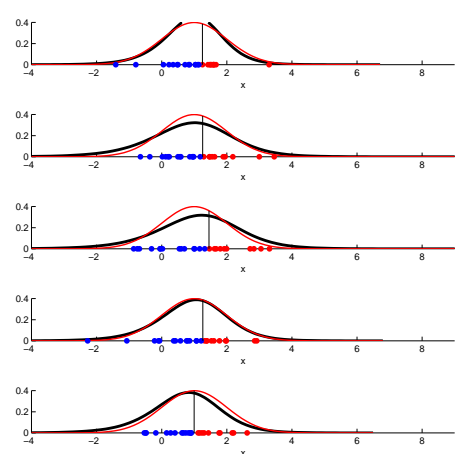

(a) $p\left(c_{\text {post }} \mid \mathcal{X}_{k, \text { post }}\right)$

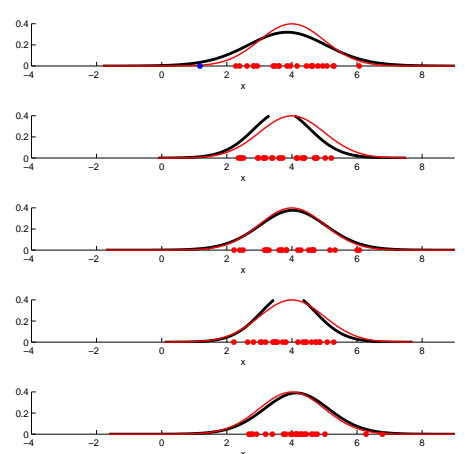

(d) $p\left(c_{\text {pre }} \mid \mathcal{X}_{k, \text { pre }}\right)$

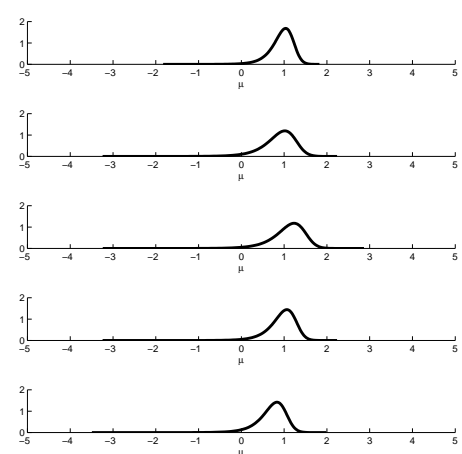

(b) $p\left(\mu_{1} \mid \mathcal{X}_{k, \text { post }}\right)$

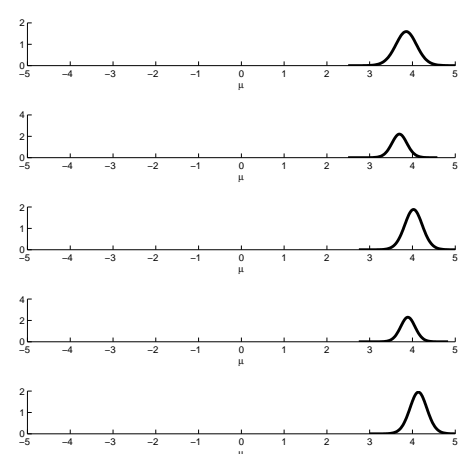

(e) $p\left(\mu_{1} \mid \mathcal{X}_{k, \text { pre }}\right)$

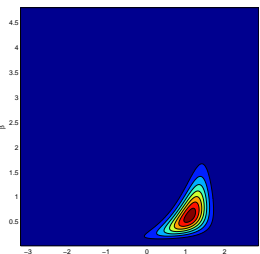

(g-3) $p\left(\mu, \beta \mid \mathcal{X}_{3, \text { post }}\right)$

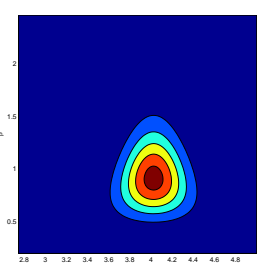

(h-3) $p\left(\mu, \beta \mid \mathcal{X}_{3, \text { pre }}\right)$

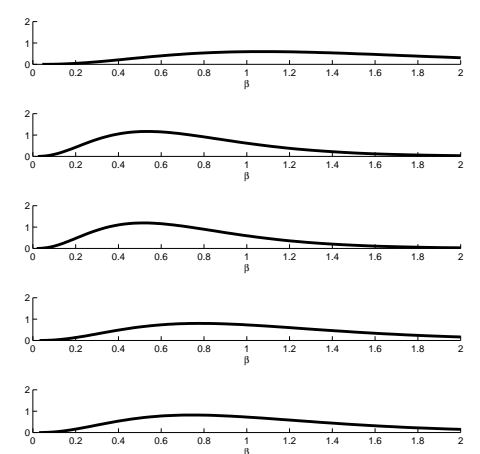

(c) $p\left(\beta_{1} \mid \mathcal{X}_{k, \text { post }}\right)$

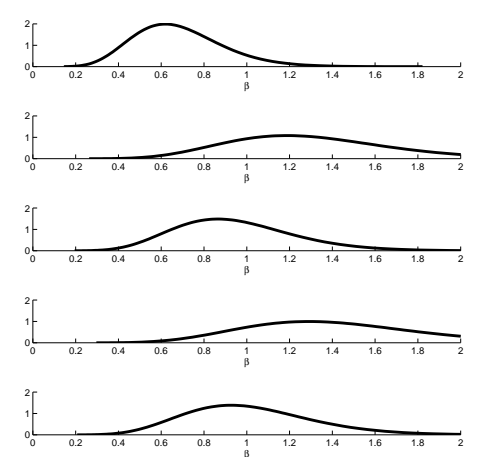

(f) $p\left(\beta_{1} \mid \mathcal{X}_{k, \text { pre }}\right)$

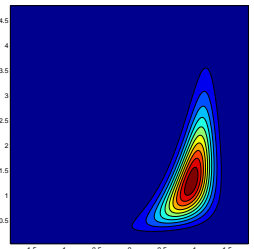

(g-1) $p\left(\mu, \beta \mid \mathcal{X}_{1, \text { post }}\right)$

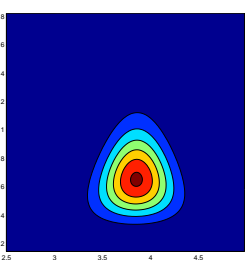

(h-1) $p\left(\mu, \beta \mid \mathcal{X}_{1, \text { pre }}\right)$

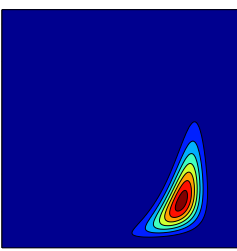

(g-2) $p\left(\mu, \beta \mid \mathcal{X}_{2, \text { post }}\right)$

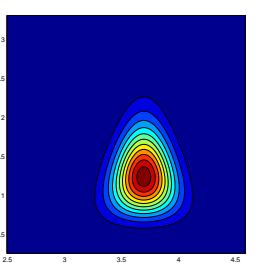

(h-2) $p\left(\mu, \beta \mid \mathcal{X}_{2, \text { pre }}\right)$

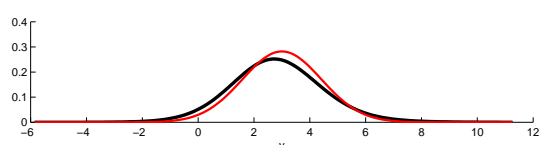

(i-2) $p\left(y \mid \mathcal{X}_{2, \text { pre }}, \mathcal{X}_{2, \text { post }}\right)$

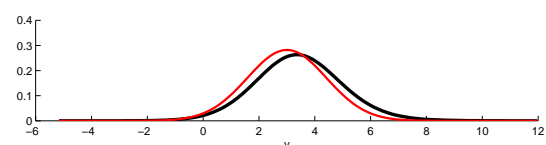

(i-5) $p\left(y \mid \mathcal{X}_{5, \text { pre }}, \mathcal{X}_{5, \text { post }}\right)$
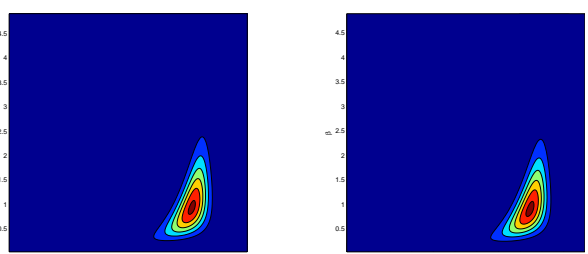

(g-4) $p\left(\mu, \beta \mid \mathcal{X}_{4, \text { post }}\right)$

(g-5) $p\left(\mu, \beta \mid \mathcal{X}_{5, \text { post }}\right)$
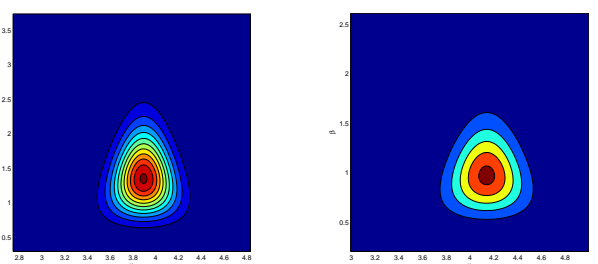

(h-4) $p\left(\mu, \beta \mid \mathcal{X}_{4, \text { pre }}\right)$

(h-5) $p\left(\mu, \beta \mid \mathcal{X}_{5, \text { pre }}\right)$

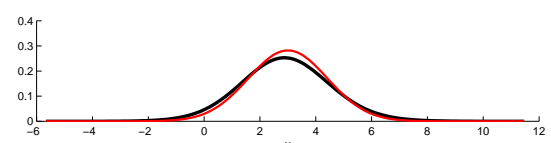

(i-1) $p\left(y \mid \mathcal{X}_{1, \text { pre }}, \mathcal{X}_{1, \text { post }}\right)$

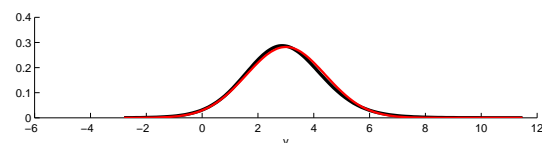

$(\mathrm{i}-4) p\left(y \mid \mathcal{X}_{4, \text { pre }}, \mathcal{X}_{4, \text { post }}\right)$

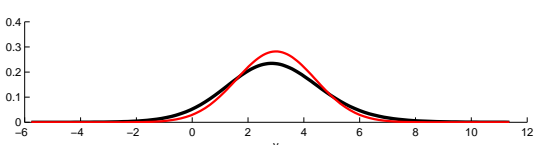

(i-3) $p\left(y \mid \mathcal{X}_{3, \text { pre }}, \mathcal{X}_{3, \text { post }}\right)$

Figure 22S: Bayesian inferences on five datasets, each of which contains $n_{v}=10$ detect data out of $n=24$ samples. 


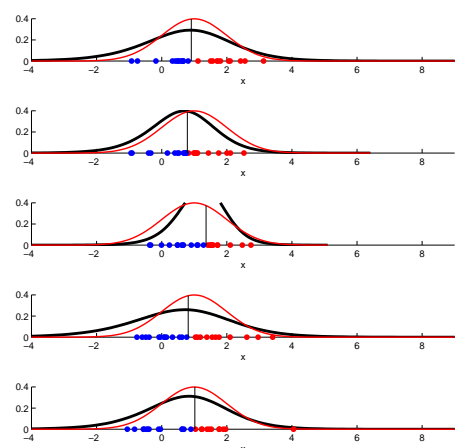

(a) $p\left(c_{\text {post }} \mid \mathcal{X}_{k, \text { post }}\right)$

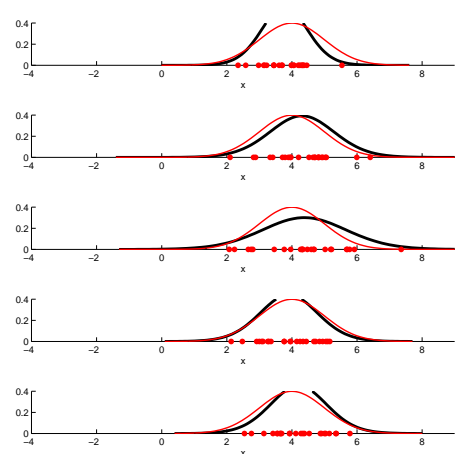

(d) $p\left(c_{\text {pre }} \mid \mathcal{X}_{k, \text { pre }}\right)$

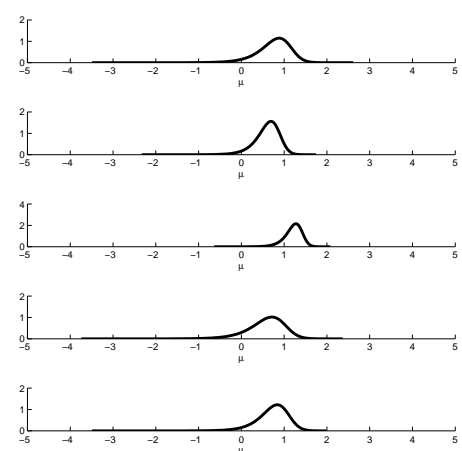

(b) $p\left(\mu_{1} \mid \mathcal{X}_{k, \text { post }}\right)$

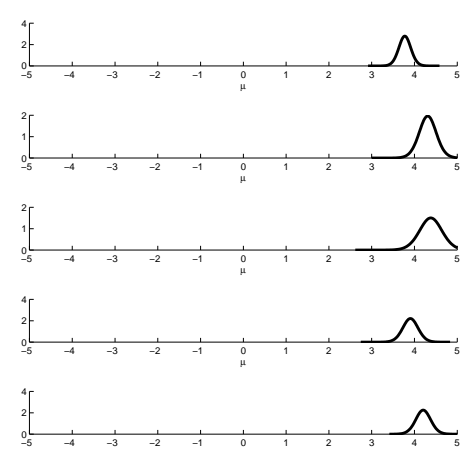

(e) $p\left(\mu_{1} \mid \mathcal{X}_{k, \text { pre }}\right)$

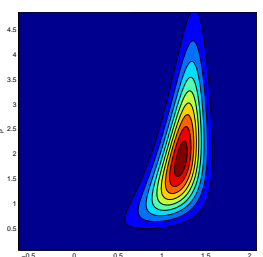

(g-3) $p\left(\mu, \beta \mid \mathcal{X}_{3, \text { post }}\right)$

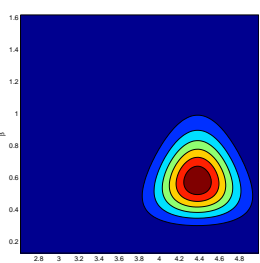

(h-3) $p\left(\mu, \beta \mid \mathcal{X}_{3, \text { pre }}\right)$

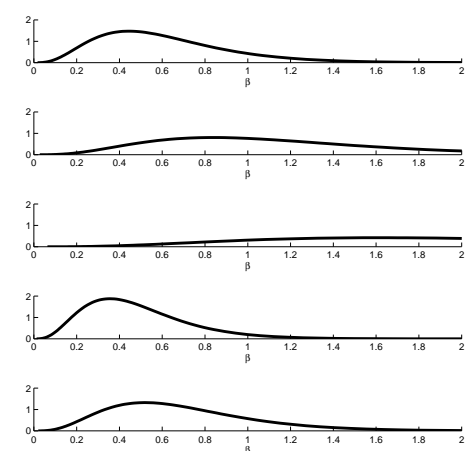

(c) $p\left(\beta_{1} \mid \mathcal{X}_{k, \text { post }}\right)$

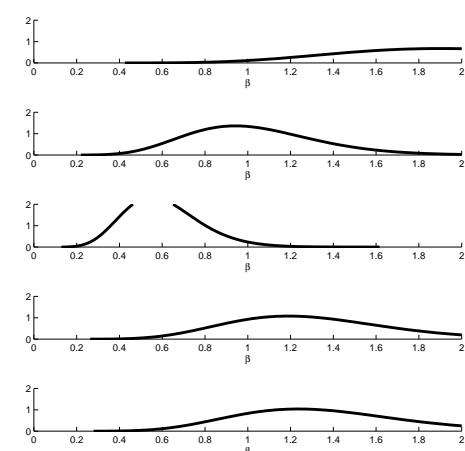

(f) $p\left(\beta_{1} \mid \mathcal{X}_{k, \text { pre }}\right)$

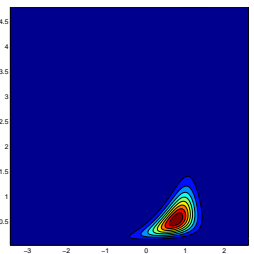

(g-1) $p\left(\mu, \beta \mid \mathcal{X}_{1, \text { post }}\right)$

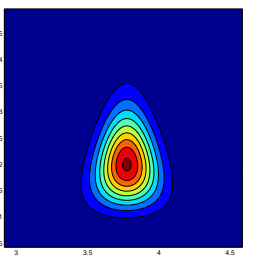

(h-1) $p\left(\mu, \beta \mid \mathcal{X}_{1, \text { pre }}\right)$

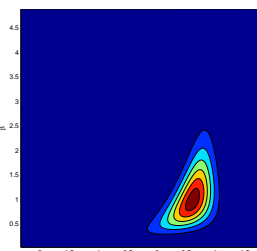

(g-2) $p\left(\mu, \beta \mid \mathcal{X}_{2, \text { post }}\right)$

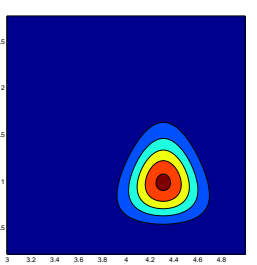

(h-2) $p\left(\mu, \beta \mid \mathcal{X}_{2, \text { pre }}\right)$

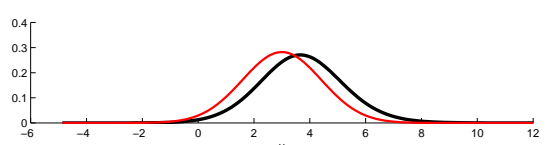

(i-2) $p\left(y \mid \mathcal{X}_{2, \text { pre }}, \mathcal{X}_{2, \text { post }}\right)$

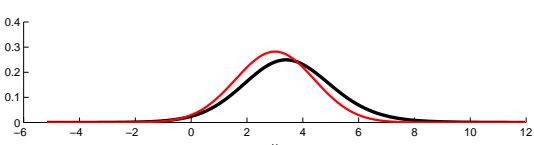

(i-5) $p\left(y \mid \mathcal{X}_{5, \text { pre }}, \mathcal{X}_{5, \text { post }}\right)$

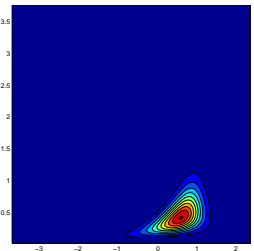

(g-4) $p\left(\mu, \beta \mid \mathcal{X}_{4, \text { post }}\right)$

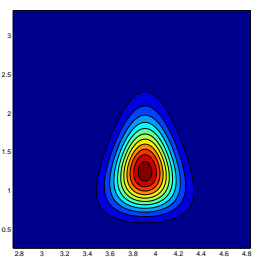

(h-4) $p\left(\mu, \beta \mid \mathcal{X}_{4, \text { pre }}\right)$

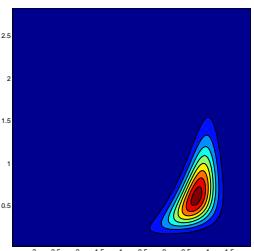

(g-5) $p\left(\mu, \beta \mid \mathcal{X}_{5, \text { post }}\right)$

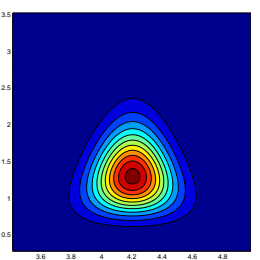

(h-5) $p\left(\mu, \beta \mid \mathcal{X}_{5, \text { pre }}\right)$

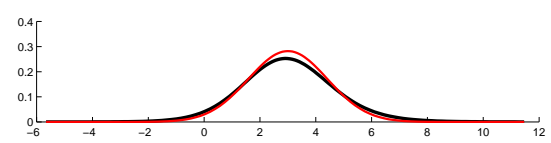

(i-1) $p\left(y \mid \mathcal{X}_{1, \text { pre }}, \mathcal{X}_{1, \text { post }}\right)$

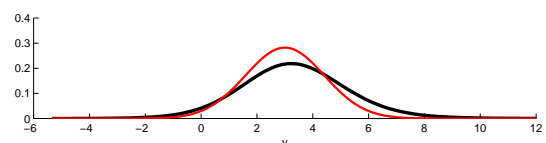

(i-4) $p\left(y \mid \mathcal{X}_{4, \text { pre }}, \mathcal{X}_{4, \text { post }}\right)$

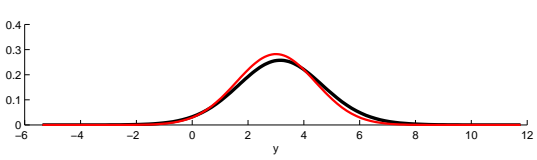

$(\mathrm{i}-3) p\left(y \mid \mathcal{X}_{3, \text { pre }}, \mathcal{X}_{3, \text { post }}\right)$

Figure 23S: Bayesian inferences on five datasets, each of which contains $n_{v}=11$ detect data out of $n=24$ samples. 


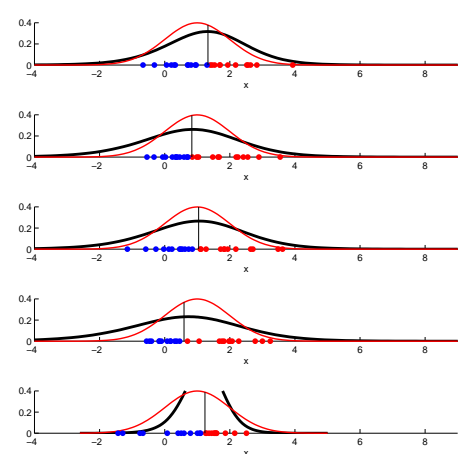

(a) $p\left(c_{\text {post }} \mid \mathcal{X}_{k, \text { post }}\right)$

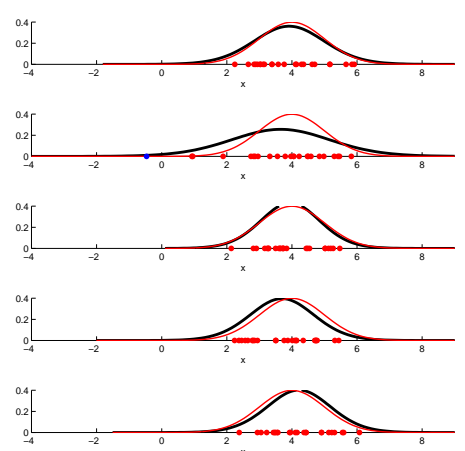

(d) $p\left(c_{\text {pre }} \mid \mathcal{X}_{k, \text { pre }}\right)$

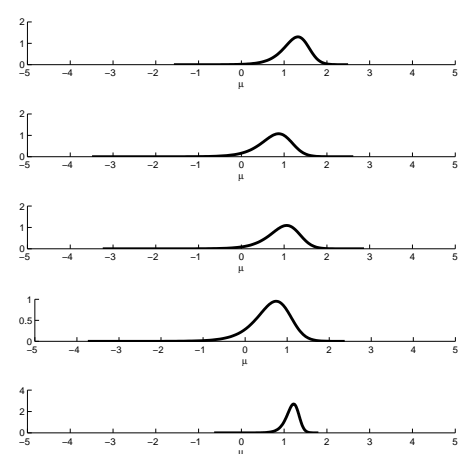

(b) $p\left(\mu_{1} \mid \mathcal{X}_{k, \text { post }}\right)$

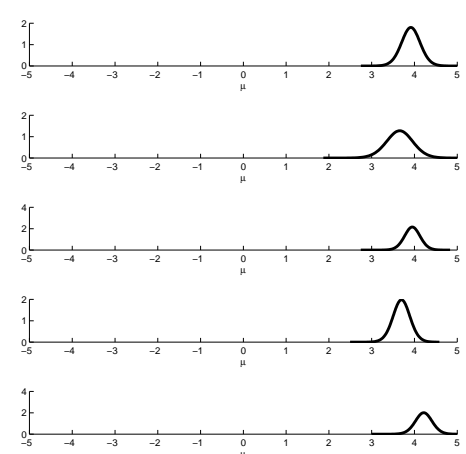

(e) $p\left(\mu_{1} \mid \mathcal{X}_{k, \text { pre }}\right)$

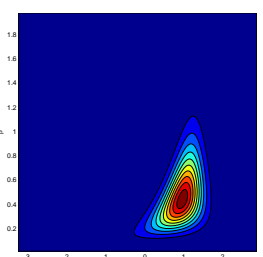

(g-3) $p\left(\mu, \beta \mid \mathcal{X}_{3, \text { post }}\right)$

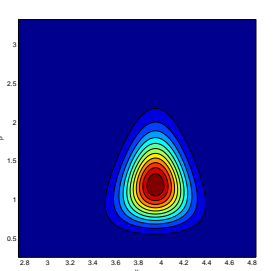

(h-3) $p\left(\mu, \beta \mid \mathcal{X}_{3, \text { pre }}\right)$

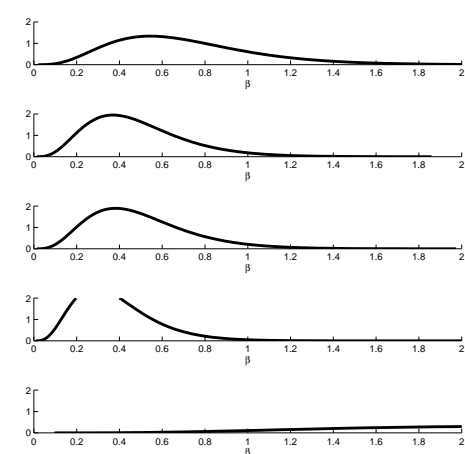

(c) $p\left(\beta_{1} \mid \mathcal{X}_{k, \text { post }}\right)$

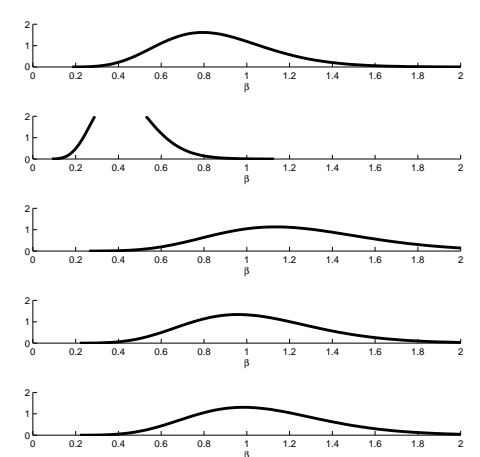

(f) $p\left(\beta_{1} \mid \mathcal{X}_{k, \text { pre }}\right)$

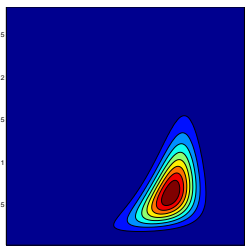

(g-1) $p\left(\mu, \beta \mid \mathcal{X}_{1, \text { post }}\right)$

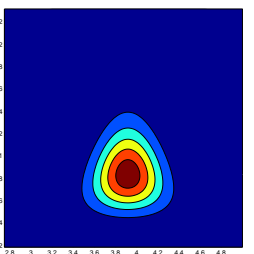

(h-1) $p\left(\mu, \beta \mid \mathcal{X}_{1, \text { pre }}\right)$

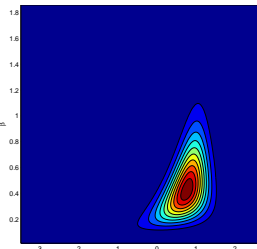

$(\mathrm{g}-2) p\left(\mu, \beta \mid \mathcal{X}_{2, \text { post }}\right)$

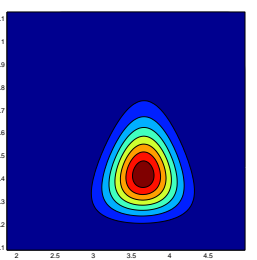

(h-2) $p\left(\mu, \beta \mid \mathcal{X}_{2, \text { pre }}\right)$

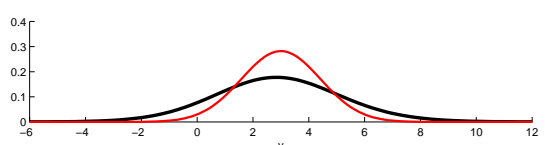

(i-2) $p\left(y \mid \mathcal{X}_{2, \text { pre }}, \mathcal{X}_{2, \text { post }}\right)$

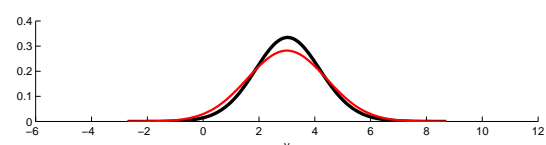

(i-5) $p\left(y \mid \mathcal{X}_{5, \text { pre }}, \mathcal{X}_{5, \text { post }}\right)$

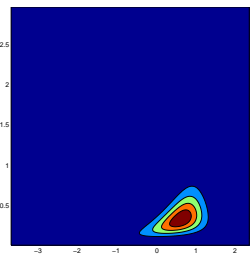

(g-4) $p\left(\mu, \beta \mid \mathcal{X}_{4, \text { post }}\right)$
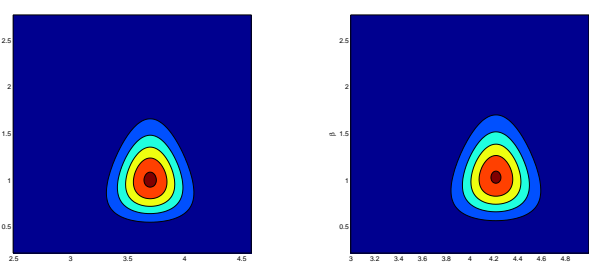

(h-4) $p\left(\mu, \beta \mid \mathcal{X}_{4, \text { pre }}\right)$

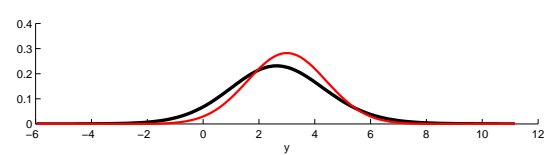

(i-1) $p\left(y \mid \mathcal{X}_{1, \text { pre }}, \mathcal{X}_{1, \text { post }}\right)$

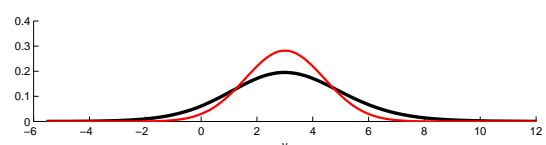

(i-4) $p\left(y \mid \mathcal{X}_{4, \text { pre }}, \mathcal{X}_{4, \text { post }}\right)$

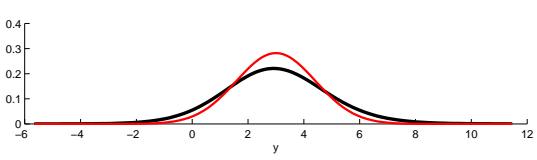

(i-3) $p\left(y \mid \mathcal{X}_{3, \text { pre }}, \mathcal{X}_{3, \text { post }}\right)$

Figure 24S: Bayesian inferences on five datasets, each of which contains $n_{v}=12$ detect data out of $n=24$ samples. 


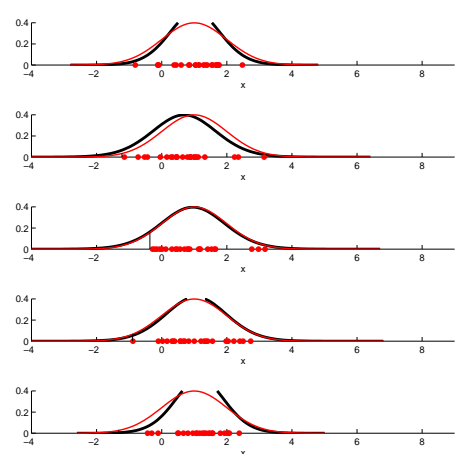

(a) $p\left(c_{\text {post }} \mid \mathcal{X}_{k, \text { post }}\right)$

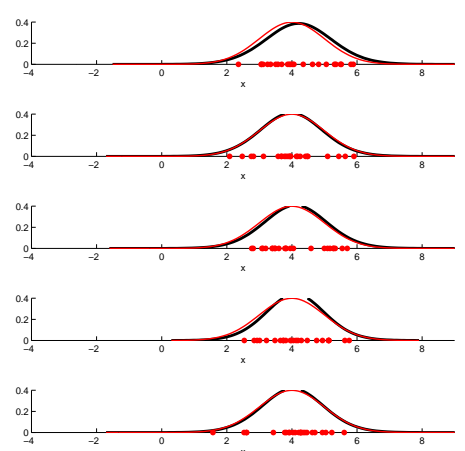

(d) $p\left(c_{\text {pre }} \mid \mathcal{X}_{k, \text { pre }}\right)$

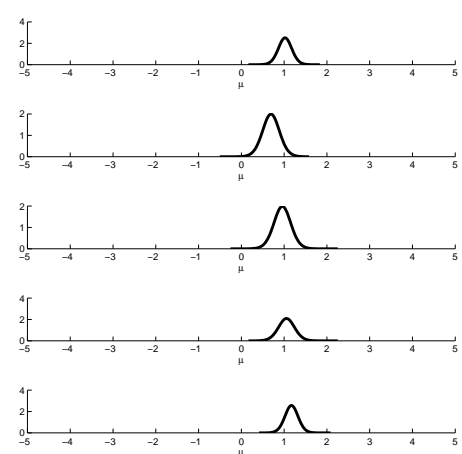

(b) $p\left(\mu_{1} \mid \mathcal{X}_{k, \text { post }}\right)$

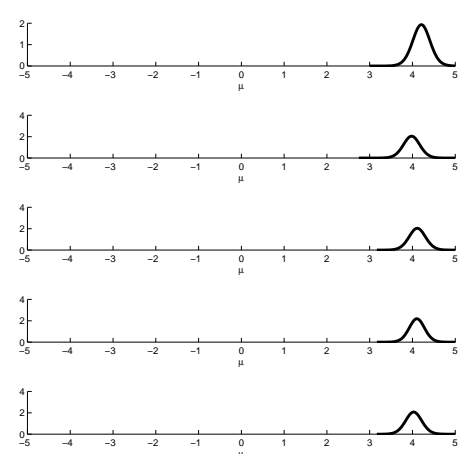

(e) $p\left(\mu_{1} \mid \mathcal{X}_{k, \text { pre }}\right)$

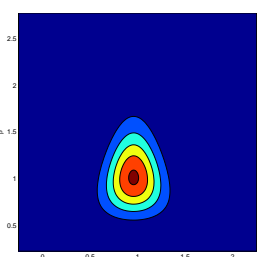

(g-3) $p\left(\mu, \beta \mid \mathcal{X}_{3, \text { post }}\right)$

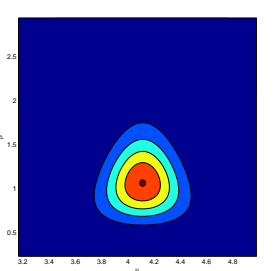

(h-3) $p\left(\mu, \beta \mid \mathcal{X}_{3, \text { pre }}\right)$

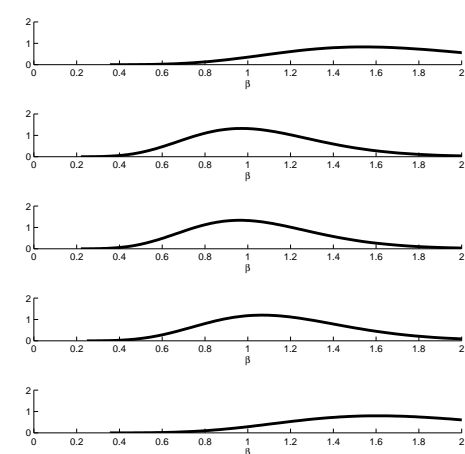

(c) $p\left(\beta_{1} \mid \mathcal{X}_{k, \text { post }}\right)$

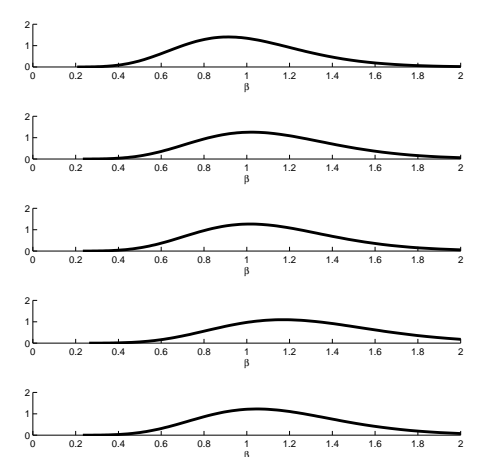

(f) $p\left(\beta_{1} \mid \mathcal{X}_{k, \text { pre }}\right)$

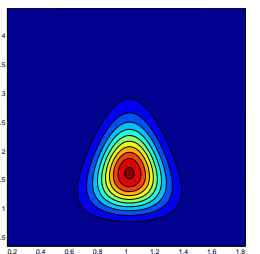

(g-1) $p\left(\mu, \beta \mid \mathcal{X}_{1, \text { post }}\right)$

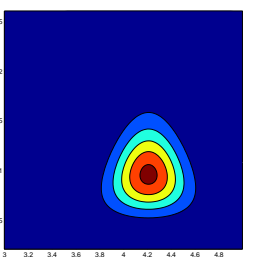

(h-1) $p\left(\mu, \beta \mid \mathcal{X}_{1, \text { pre }}\right)$

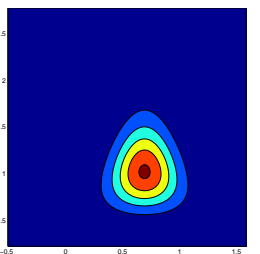

(g-2) $p\left(\mu, \beta \mid \mathcal{X}_{2, \text { post }}\right)$

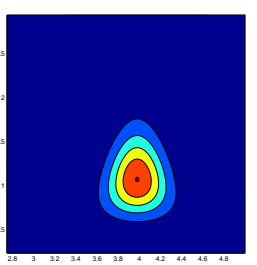

(h-2) $p\left(\mu, \beta \mid \mathcal{X}_{2, \text { pre }}\right)$

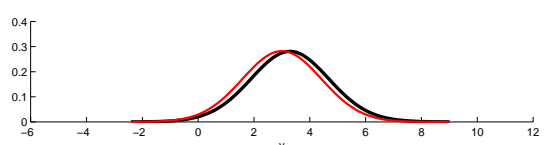

(i-2) $p\left(y \mid \mathcal{X}_{2, \text { pre }}, \mathcal{X}_{2, \text { post }}\right)$

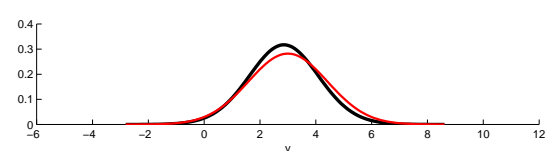

(i-5) $p\left(y \mid \mathcal{X}_{5, \text { pre }}, \mathcal{X}_{5, \text { post }}\right)$

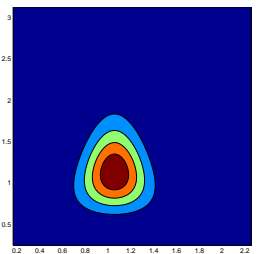

(g-4) $p\left(\mu, \beta \mid \mathcal{X}_{4, \text { post }}\right)$
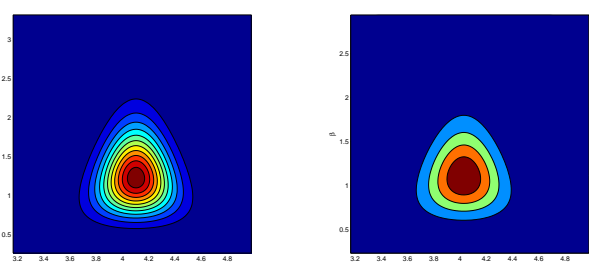

(h-4) $p\left(\mu, \beta \mid \mathcal{X}_{4, \text { pre }}\right)$

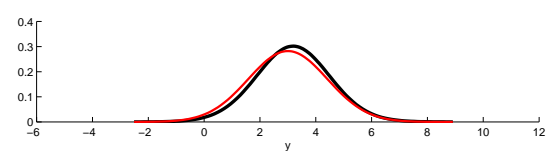

(i-1) $p\left(y \mid \mathcal{X}_{1, \text { pre }}, \mathcal{X}_{1, \text { post }}\right)$

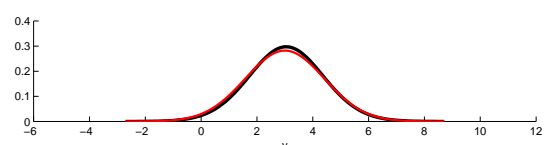

(i-4) $p\left(y \mid \mathcal{X}_{4, \text { pre }}, \mathcal{X}_{4, \text { post }}\right)$

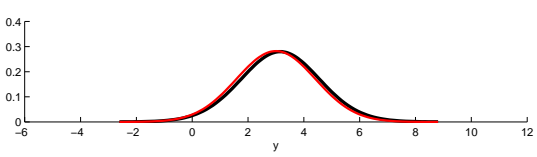

(i-3) $p\left(y \mid \mathcal{X}_{3, \text { pre }}, \mathcal{X}_{3, \text { post }}\right)$

Figure 25S: Bayesian inferences on five datasets, each of which contains $n_{v}=24$ detect data out of $n=24$ samples. 


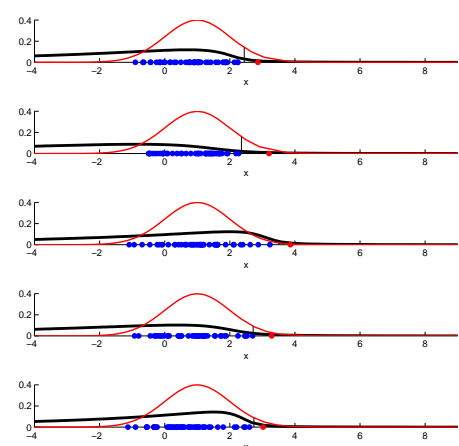

(a) $p\left(c_{\text {post }} \mid \mathcal{X}_{k, \text { post }}\right)$

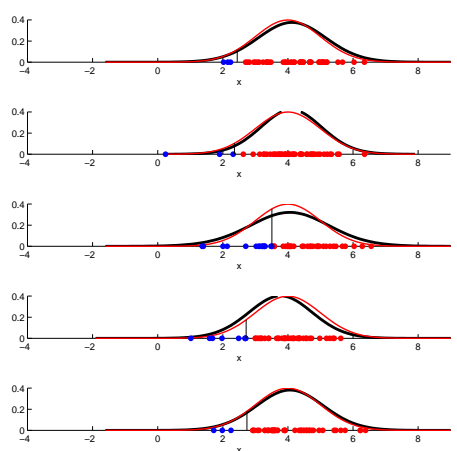

(d) $p\left(c_{\text {pre }} \mid \mathcal{X}_{k, \text { pre }}\right)$

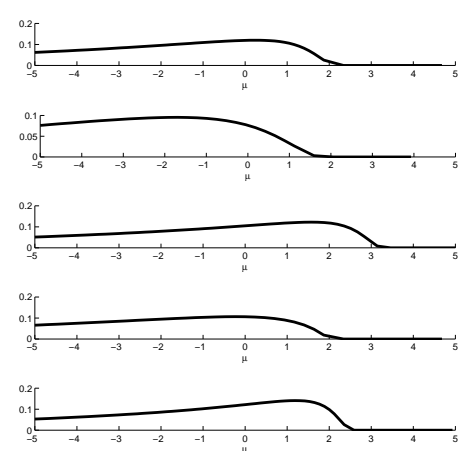

(b) $p\left(\mu_{1} \mid \mathcal{X}_{k, \text { post }}\right)$

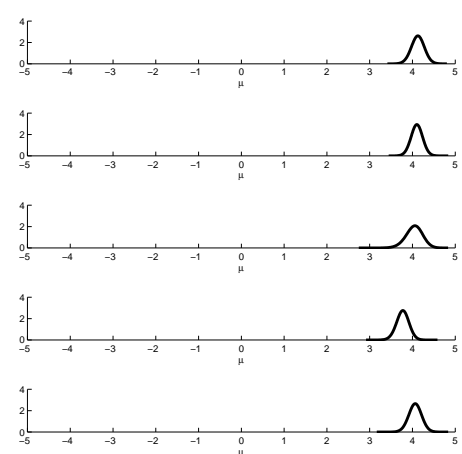

(e) $p\left(\mu_{1} \mid \mathcal{X}_{k, \text { pre }}\right)$

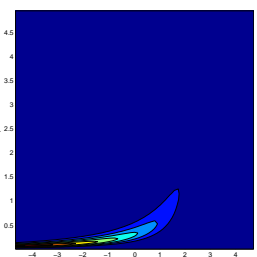

(g-3) $p\left(\mu, \beta \mid \mathcal{X}_{3, \text { post }}\right)$

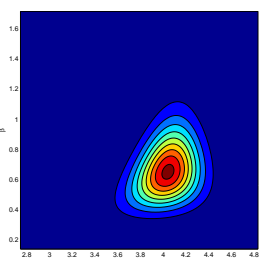

(h-3) $p\left(\mu, \beta \mid \mathcal{X}_{3, \text { pre }}\right)$

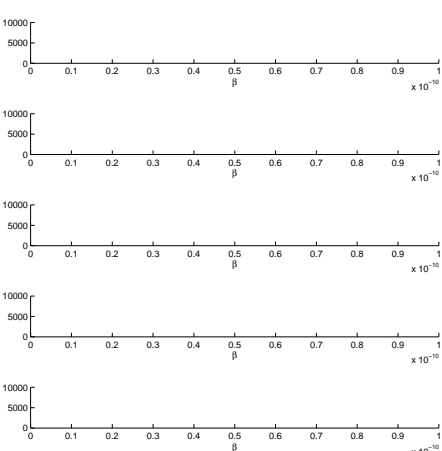

(c) $p\left(\beta_{1} \mid \mathcal{X}_{k, \text { post }}\right)$

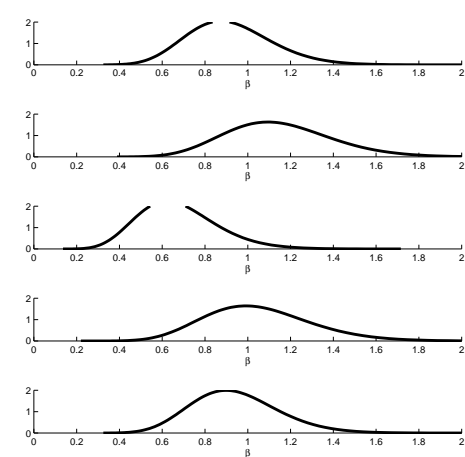

(f) $p\left(\beta_{1} \mid \mathcal{X}_{k, \text { pre }}\right)$

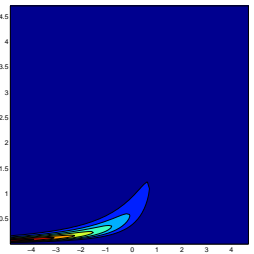

(g-1) $p\left(\mu, \beta \mid \mathcal{X}_{1, \text { post }}\right)$

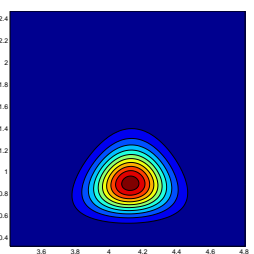

(h-1) $p\left(\mu, \beta \mid \mathcal{X}_{1, \text { pre }}\right)$

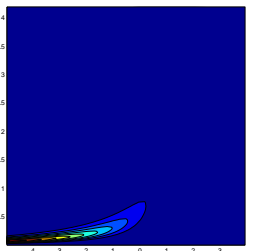

(g-2) $p\left(\mu, \beta \mid \mathcal{X}_{2, \text { post }}\right)$

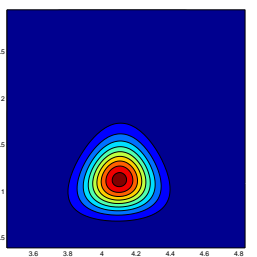

(h-2) $p\left(\mu, \beta \mid \mathcal{X}_{2, \text { pre }}\right)$

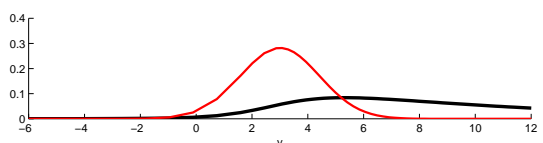

(i-2) $p\left(y \mid \mathcal{X}_{2, \text { pre }}, \mathcal{X}_{2, \text { post }}\right)$

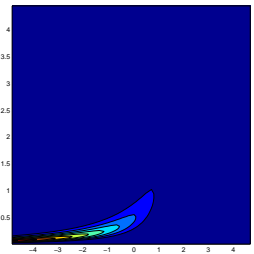

(g-4) $p\left(\mu, \beta \mid \mathcal{X}_{4, \text { post }}\right)$

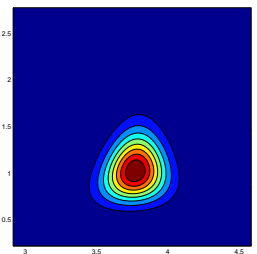

(h-4) $p\left(\mu, \beta \mid \mathcal{X}_{4, \text { pre }}\right)$

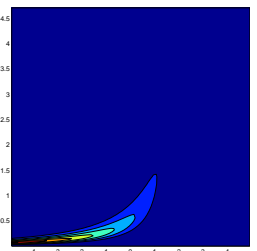

(g-5) $p\left(\mu, \beta \mid \mathcal{X}_{5, \text { post }}\right)$

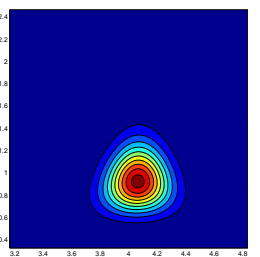

(h-5) $p\left(\mu, \beta \mid \mathcal{X}_{5, \text { pre }}\right)$

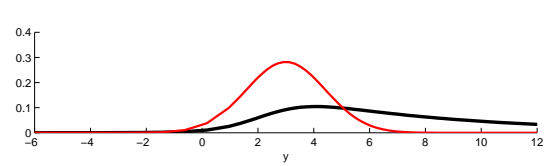

(i-1) $p\left(y \mid \mathcal{X}_{1, \text { pre }}, \mathcal{X}_{1, \text { post }}\right)$

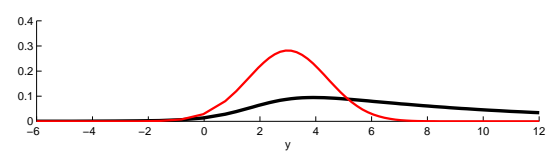

(i-4) $p\left(y \mid \mathcal{X}_{4, \text { pre }}, \mathcal{X}_{4, \text { post }}\right)$

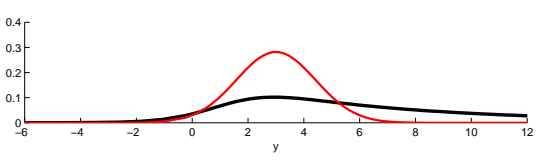

(i-3) $p\left(y \mid \mathcal{X}_{3, \text { pre }}, \mathcal{X}_{3, \text { post }}\right)$

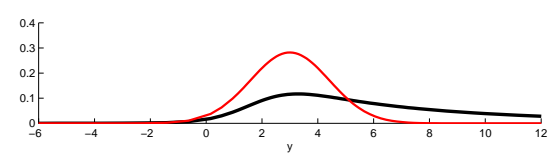

$(\mathrm{i}-5) p\left(y \mid \mathcal{X}_{5, \text { pre }}, \mathcal{X}_{5, \text { post }}\right)$

Figure 26S: Bayesian inferences on five datasets, each of which contains $n_{v}=1$ detect data out of $n=48$ samples. 


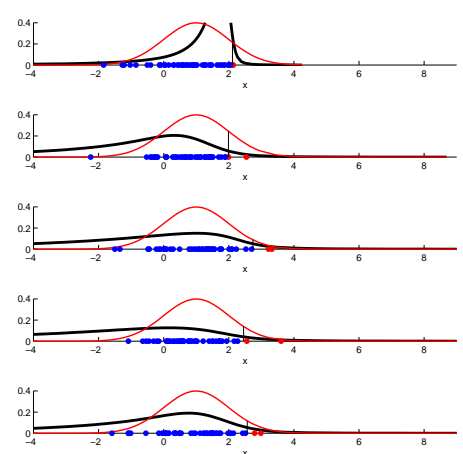

(a) $p\left(c_{\text {post }} \mid \mathcal{X}_{k \text {,post }}\right)$

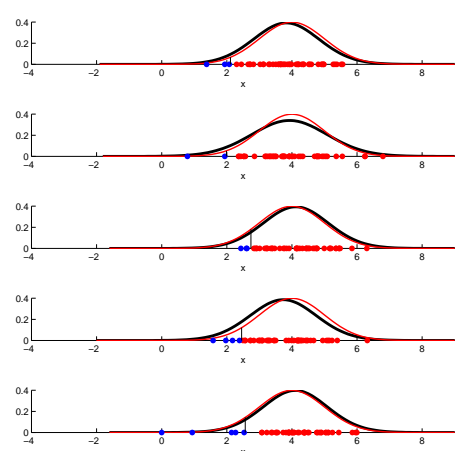

(d) $p\left(c_{\text {pre }} \mid \mathcal{X}_{k, \text { pre }}\right)$

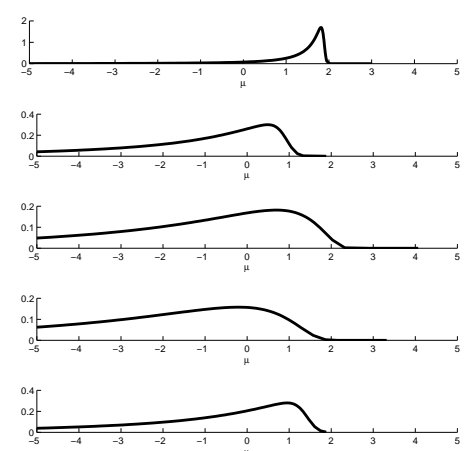

(b) $p\left(\mu_{1} \mid \mathcal{X}_{k, \text { post }}\right)$

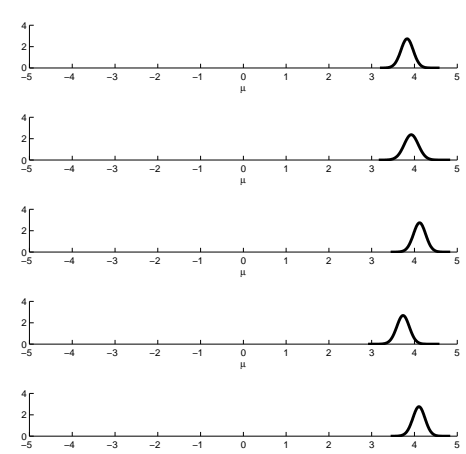

(e) $p\left(\mu_{1} \mid \mathcal{X}_{k, \text { pre }}\right)$

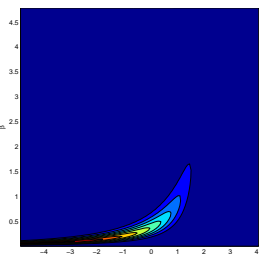

(g-3) $p\left(\mu, \beta \mid \mathcal{X}_{3, \text { post }}\right)$

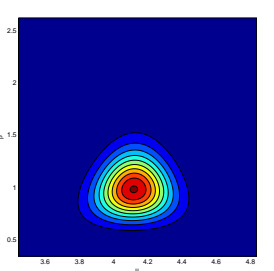

(h-3) $p\left(\mu, \beta \mid \mathcal{X}_{3, \text { pre }}\right)$

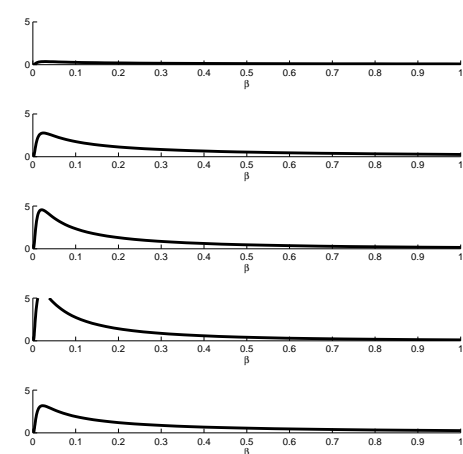

(c) $p\left(\beta_{1} \mid \mathcal{X}_{k, \text { post }}\right)$

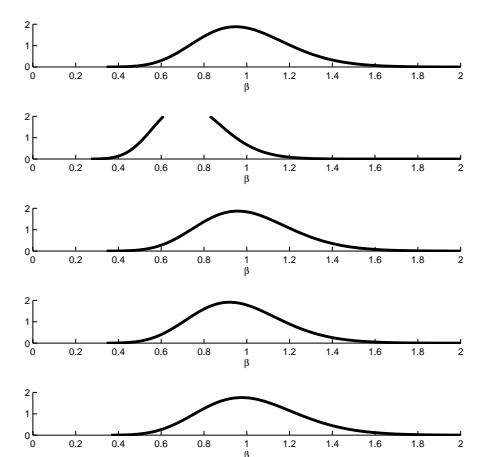

(f) $p\left(\beta_{1} \mid \mathcal{X}_{k, \text { pre }}\right)$

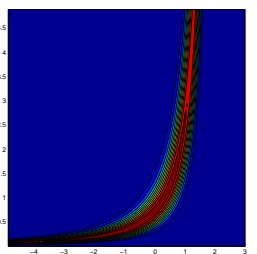

(g-1) $p\left(\mu, \beta \mid \mathcal{X}_{1, \text { post }}\right)$

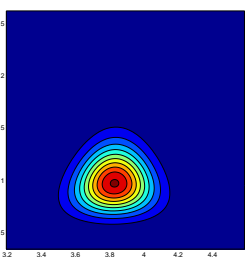

(h-1) $p\left(\mu, \beta \mid \mathcal{X}_{1, \text { pre }}\right)$

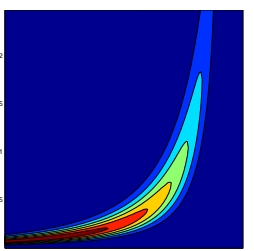

(g-2) $p\left(\mu, \beta \mid \mathcal{X}_{2, \text { post }}\right)$

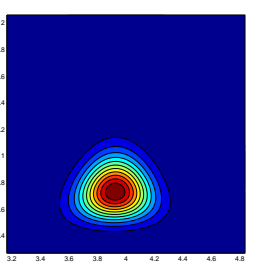

(h-2) $p\left(\mu, \beta \mid \mathcal{X}_{2, \text { pre }}\right)$

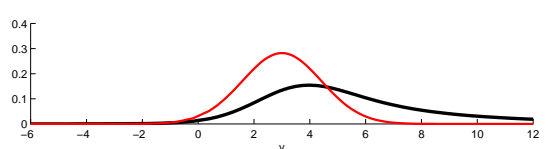

(i-2) $p\left(y \mid \mathcal{X}_{2, \text { pre }}, \mathcal{X}_{2, \text { post }}\right)$

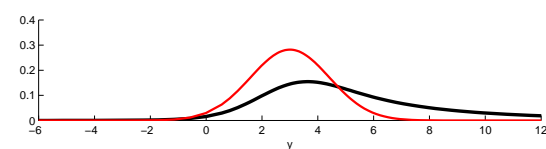

(i-5) $p\left(y \mid \mathcal{X}_{5, \text { pre }}, \mathcal{X}_{5, \text { post }}\right)$

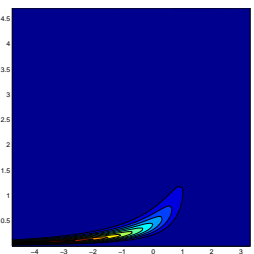

(g-4) $p\left(\mu, \beta \mid \mathcal{X}_{4, \text { post }}\right)$
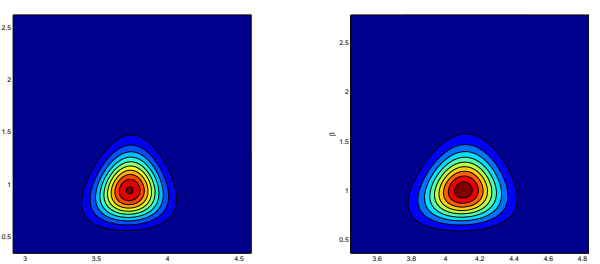

(h-4) $p\left(\mu, \beta \mid \mathcal{X}_{4, \text { pre }}\right)$

(h-5) $p\left(\mu, \beta \mid \mathcal{X}_{5, \text { pre }}\right)$

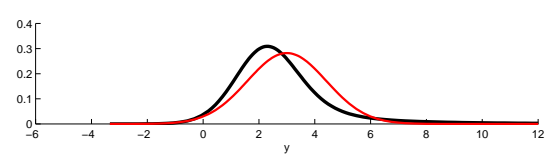

(i-1) $p\left(y \mid \mathcal{X}_{1, \text { pre }}, \mathcal{X}_{1, \text { post }}\right)$

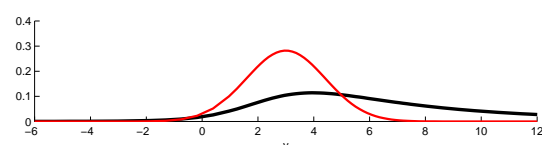

(i-4) $p\left(y \mid \mathcal{X}_{4, \text { pre }}, \mathcal{X}_{4, \text { post }}\right)$

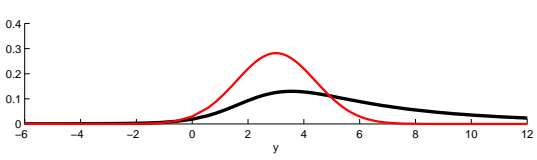

(i-3) $p\left(y \mid \mathcal{X}_{3, \text { pre }}, \mathcal{X}_{3, \text { post }}\right)$

Figure 27S: Bayesian inferences on five datasets, each of which contains $n_{v}=2$ detect data out of $n=48$ samples. 


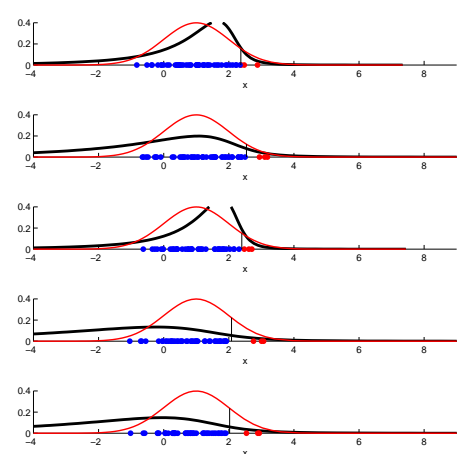

(a) $p\left(c_{\text {post }} \mid \mathcal{X}_{k \text {,post }}\right)$

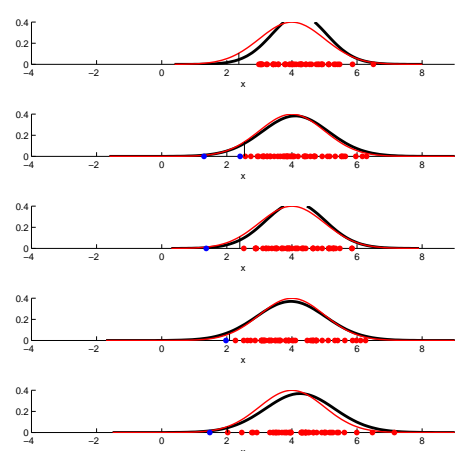

(d) $p\left(c_{\text {pre }} \mid \mathcal{X}_{k, \text { pre }}\right)$

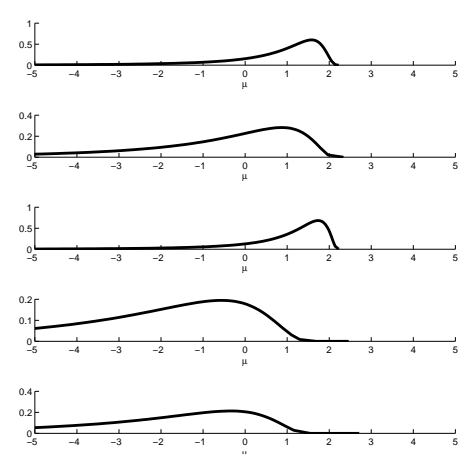

(b) $p\left(\mu_{1} \mid \mathcal{X}_{k, \text { post }}\right)$

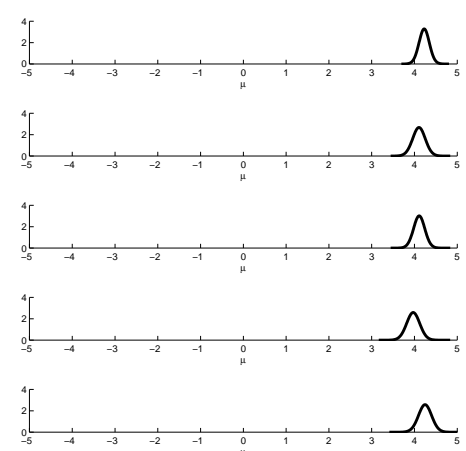

(e) $p\left(\mu_{1} \mid \mathcal{X}_{k, \text { pre }}\right)$

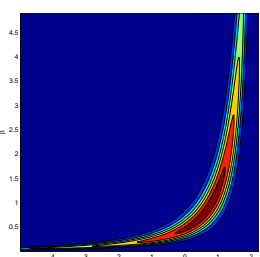

(g-3) $p\left(\mu, \beta \mid \mathcal{X}_{3, \text { post }}\right)$

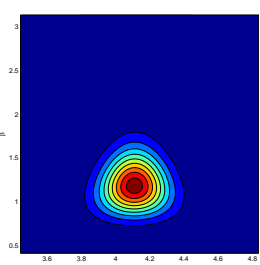

(h-3) $p\left(\mu, \beta \mid \mathcal{X}_{3, \text { pre }}\right)$

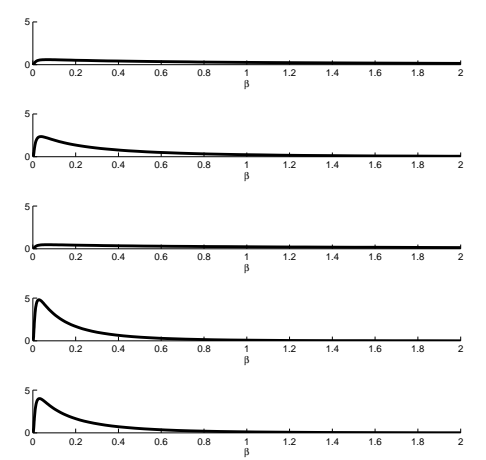

(c) $p\left(\beta_{1} \mid \mathcal{X}_{k, \text { post }}\right)$

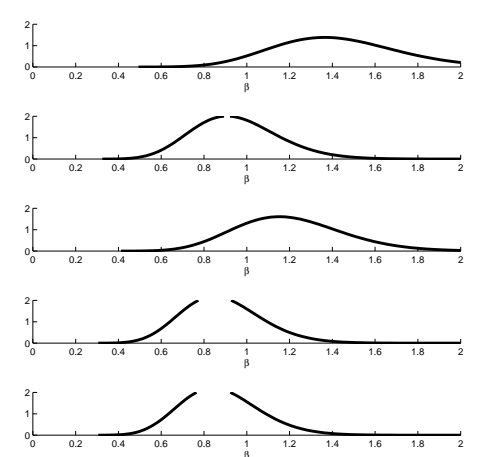

(f) $p\left(\beta_{1} \mid \mathcal{X}_{k, \text { pre }}\right)$

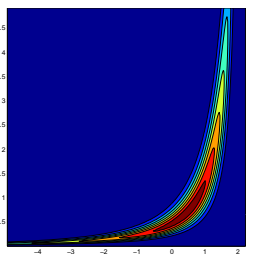

(g-1) $p\left(\mu, \beta \mid \mathcal{X}_{1, \text { post }}\right)$

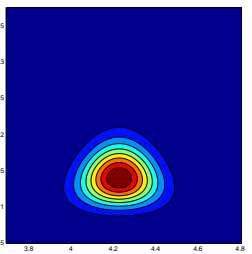

(h-1) $p\left(\mu, \beta \mid \mathcal{X}_{1, \text { pre }}\right)$

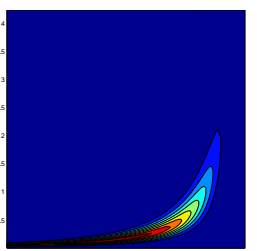

(g-2) $p\left(\mu, \beta \mid \mathcal{X}_{2, \text { post }}\right)$

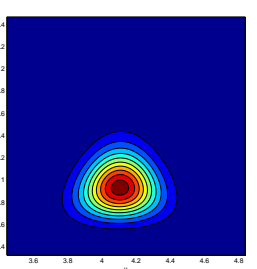

(h-2) $p\left(\mu, \beta \mid \mathcal{X}_{2, \text { pre }}\right)$

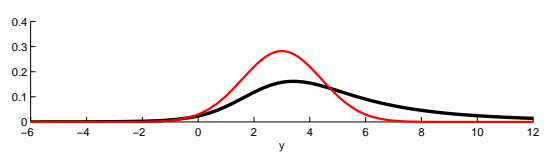

(i-2) $p\left(y \mid \mathcal{X}_{2, \text { pre }}, \mathcal{X}_{2, \text { post }}\right)$

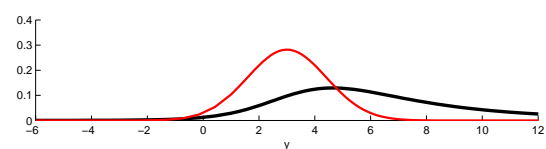

(i-5) $p\left(y \mid \mathcal{X}_{5, \text { pre }}, \mathcal{X}_{5, \text { post }}\right)$

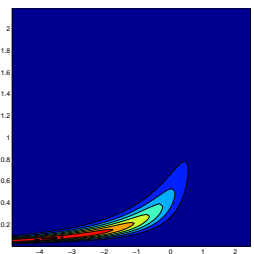

(g-4) $p\left(\mu, \beta \mid \mathcal{X}_{4, \text { post }}\right)$

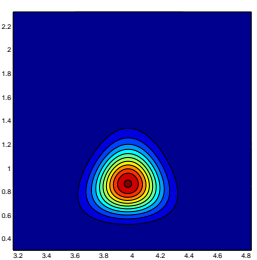

(h-4) $p\left(\mu, \beta \mid \mathcal{X}_{4, \text { pre }}\right)$

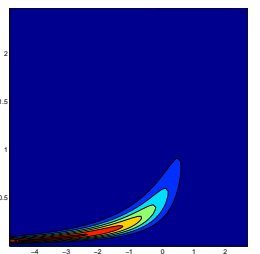

(g-5) $p\left(\mu, \beta \mid \mathcal{X}_{5, \text { post }}\right)$

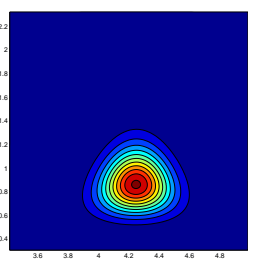

(h-5) $p\left(\mu, \beta \mid \mathcal{X}_{5, \text { pre }}\right)$

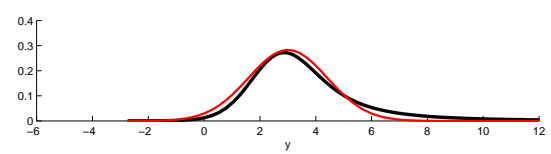

(i-1) $p\left(y \mid \mathcal{X}_{1, \text { pre }}, \mathcal{X}_{1, \text { post }}\right)$

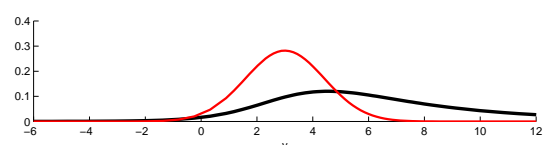

(i-4) $p\left(y \mid \mathcal{X}_{4, \text { pre }}, \mathcal{X}_{4, \text { post }}\right)$

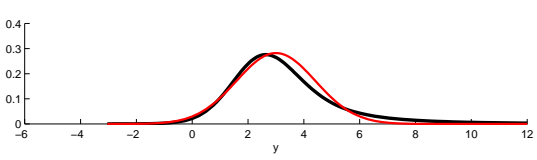

(i-3) $p\left(y \mid \mathcal{X}_{3, \text { pre }}, \mathcal{X}_{3, \text { post }}\right)$

Figure 28S: Bayesian inferences on five datasets, each of which contains $n_{v}=3$ detect data out of $n=48$ samples. 


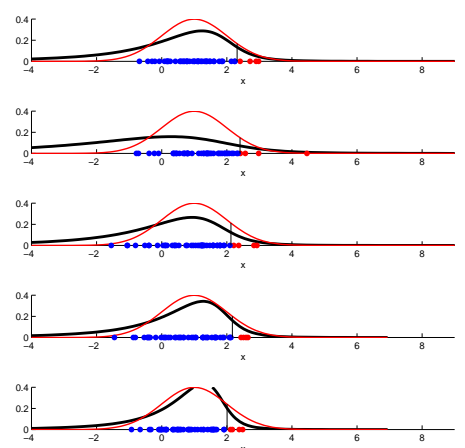

(a) $p\left(c_{\text {post }} \mid \mathcal{X}_{k, \text { post }}\right)$

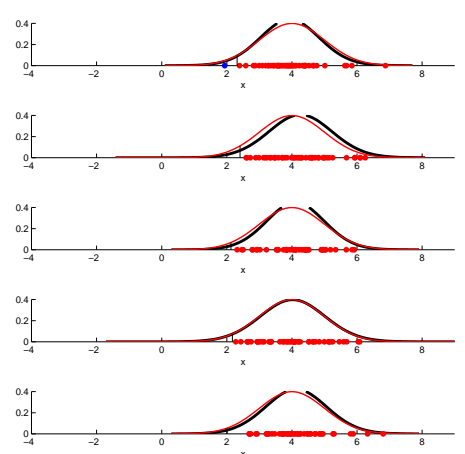

(d) $p\left(c_{\text {pre }} \mid \mathcal{X}_{k, \text { pre }}\right)$

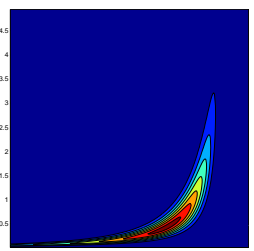

(g-1) $p\left(\mu, \beta \mid \mathcal{X}_{1, \text { post }}\right)$

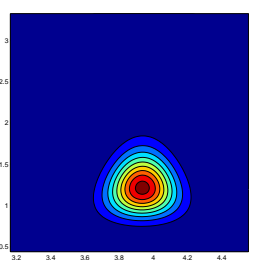

(h-1) $p\left(\mu, \beta \mid \mathcal{X}_{1, \text { pre }}\right)$

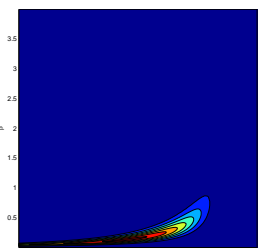

(g-2) $p\left(\mu, \beta \mid \mathcal{X}_{2, \text { post }}\right)$

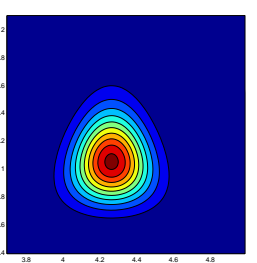

(h-2) $p\left(\mu, \beta \mid \mathcal{X}_{2, \text { pre }}\right)$

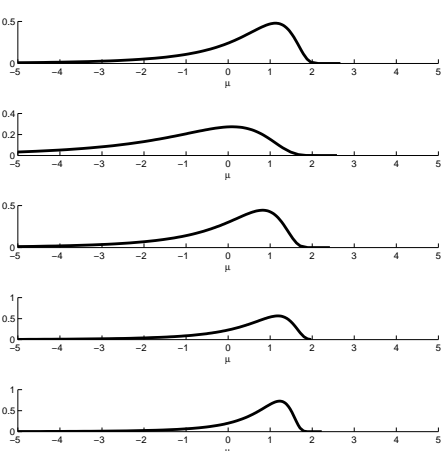

(b) $p\left(\mu_{1} \mid \mathcal{X}_{k, \text { post }}\right)$

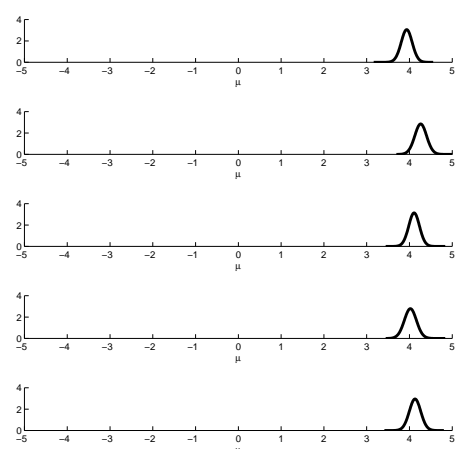

(e) $p\left(\mu_{1} \mid \mathcal{X}_{k, \text { pre }}\right)$

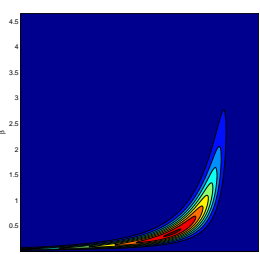

(g-3) $p\left(\mu, \beta \mid \mathcal{X}_{3, \text { post }}\right)$

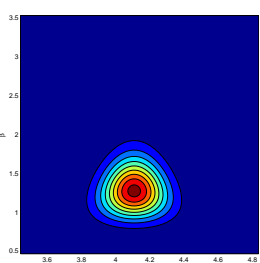

(h-3) $p\left(\mu, \beta \mid \mathcal{X}_{3, \text { pre }}\right)$

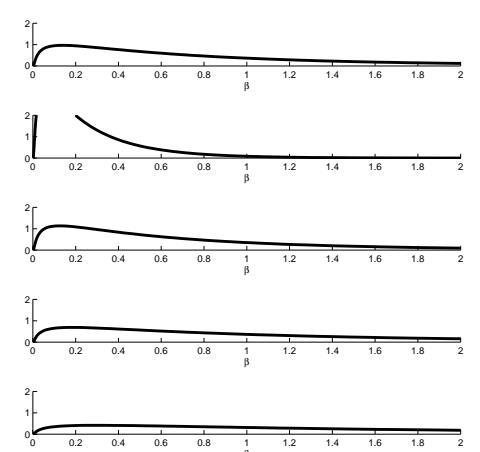

(c) $p\left(\beta_{1} \mid \mathcal{X}_{k, \text { post }}\right)$

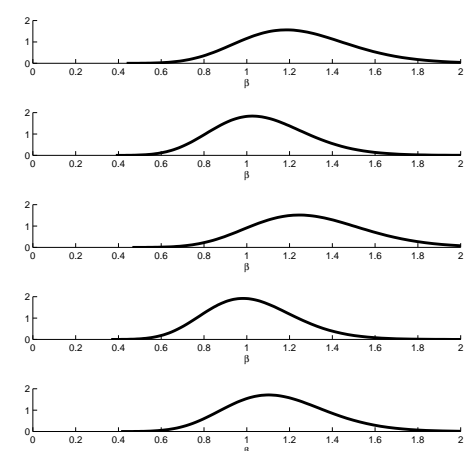

(f) $p\left(\beta_{1} \mid \mathcal{X}_{k, \text { pre }}\right)$

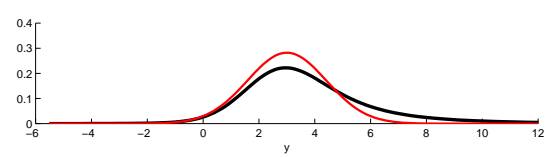

(i-1) $p\left(y \mid \mathcal{X}_{1, \text { pre }}, \mathcal{X}_{1, \text { post }}\right)$

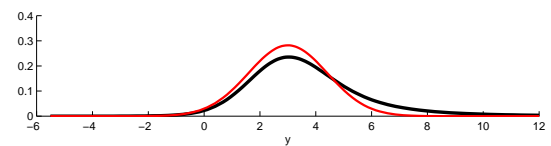

(i-4) $p\left(y \mid \mathcal{X}_{4, \text { pre }}, \mathcal{X}_{4, \text { post }}\right)$

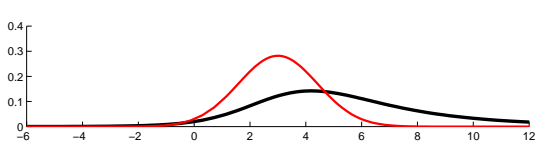

(i-2) $p\left(y \mid \mathcal{X}_{2, \text { pre }}, \mathcal{X}_{2, \text { post }}\right)$

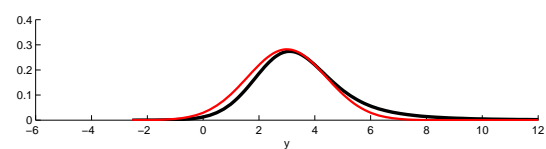

(i-5) $p\left(y \mid \mathcal{X}_{5, \text { pre }}, \mathcal{X}_{5, \text { post }}\right)$
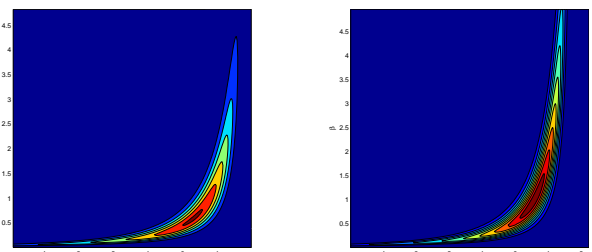

(g-4) $p\left(\mu, \beta \mid \mathcal{X}_{4, \text { post }}\right)$

(g-5) $p\left(\mu, \beta \mid \mathcal{X}_{5, \text { post }}\right)$
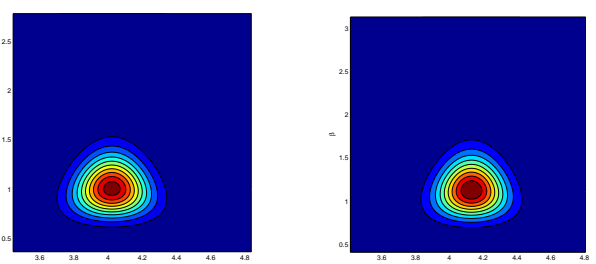

(h-4) $p\left(\mu, \beta \mid \mathcal{X}_{4, \text { pre }}\right)$

(h-5) $p\left(\mu, \beta \mid \mathcal{X}_{5, \text { pre }}\right)$

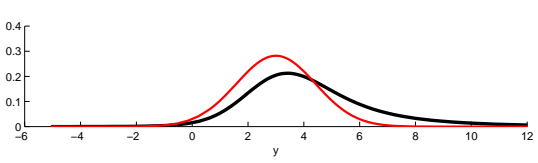

$(\mathrm{i}-3) p\left(y \mid \mathcal{X}_{3, \text { pre }}, \mathcal{X}_{3, \text { post }}\right)$

Figure 29S: Bayesian inferences on five datasets, each of which contains $n_{v}=4$ detect data out of $n=48$ samples. 


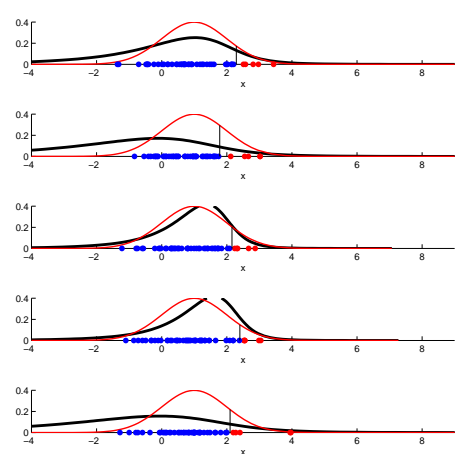

(a) $p\left(c_{\text {post }} \mid \mathcal{X}_{k, \text { post }}\right)$

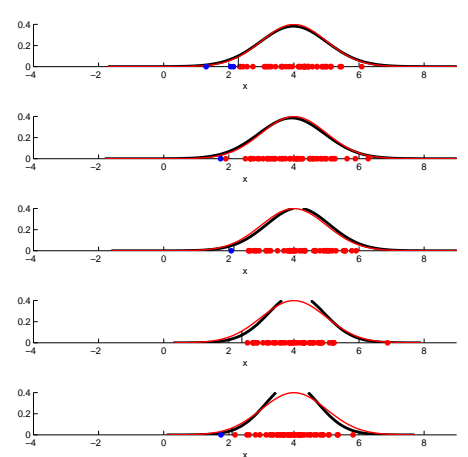

(d) $p\left(c_{\text {pre }} \mid \mathcal{X}_{k, \text { pre }}\right)$

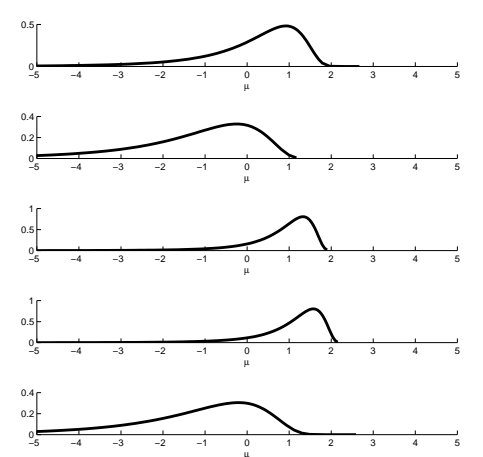

(b) $p\left(\mu_{1} \mid \mathcal{X}_{k, \text { post }}\right)$

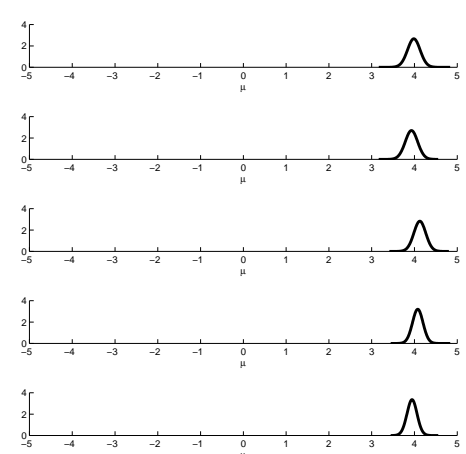

(e) $p\left(\mu_{1} \mid \mathcal{X}_{k, \text { pre }}\right)$

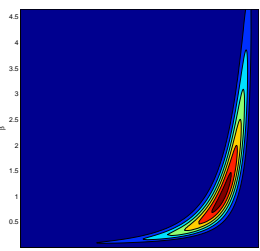

$(\mathrm{g}-3) p\left(\mu, \beta \mid \mathcal{X}_{3, \text { post }}\right)$

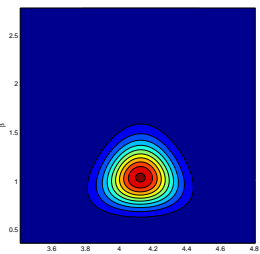

(h-3) $p\left(\mu, \beta \mid \mathcal{X}_{3, \text { pre }}\right)$

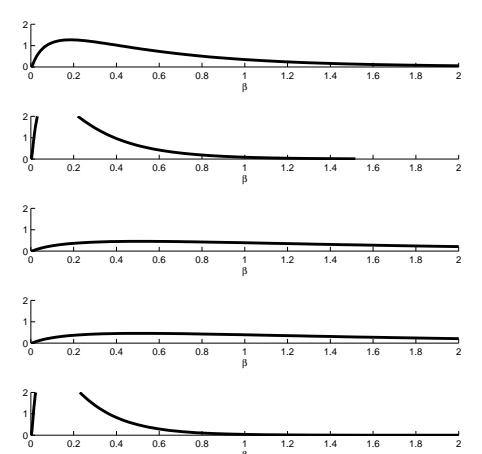

(c) $p\left(\beta_{1} \mid \mathcal{X}_{k, \text { post }}\right)$

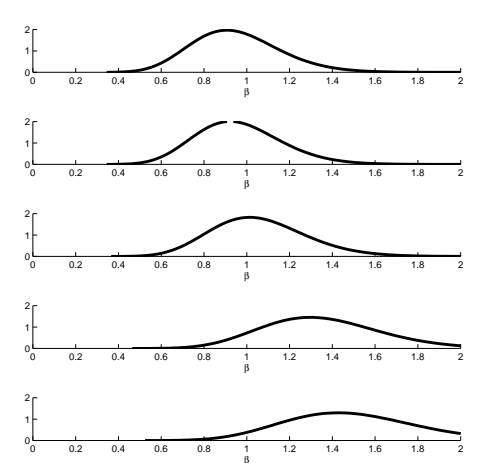

(f) $p\left(\beta_{1} \mid \mathcal{X}_{k, \text { pre }}\right)$

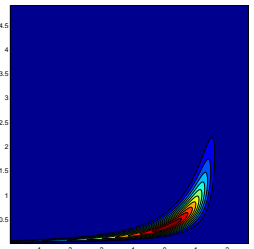

(g-1) $p\left(\mu, \beta \mid \mathcal{X}_{1, \text { post }}\right)$

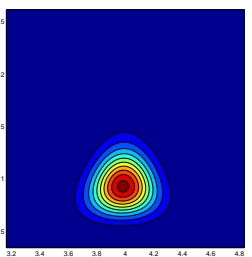

(h-1) $p\left(\mu, \beta \mid \mathcal{X}_{1, \text { pre }}\right)$

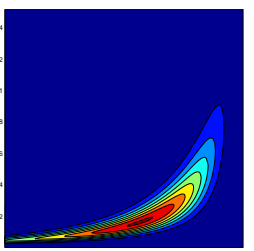

(g-2) $p\left(\mu, \beta \mid \mathcal{X}_{2, \text { post }}\right)$

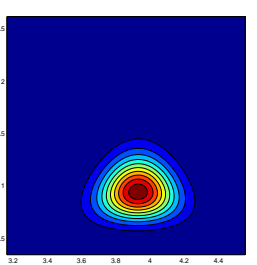

(h-2) $p\left(\mu, \beta \mid \mathcal{X}_{2, \text { pre }}\right)$

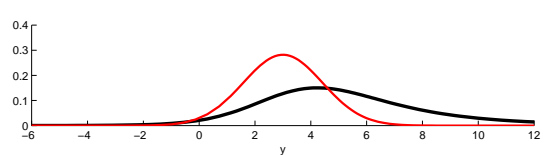

(i-2) $p\left(y \mid \mathcal{X}_{2, \text { pre }}, \mathcal{X}_{2, \text { post }}\right)$

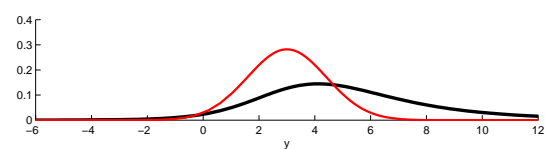

(i-5) $p\left(y \mid \mathcal{X}_{5, \text { pre }}, \mathcal{X}_{5, \text { post }}\right)$
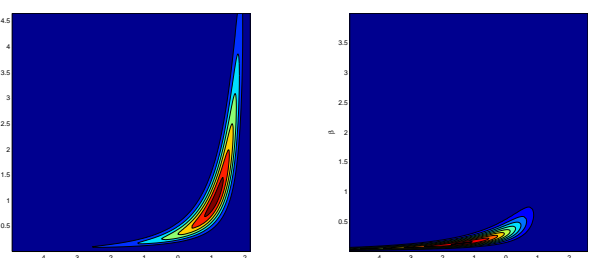

(g-4) $p\left(\mu, \beta \mid \mathcal{X}_{4, \text { post }}\right)$

(g-5) $p\left(\mu, \beta \mid \mathcal{X}_{5, \text { post }}\right)$
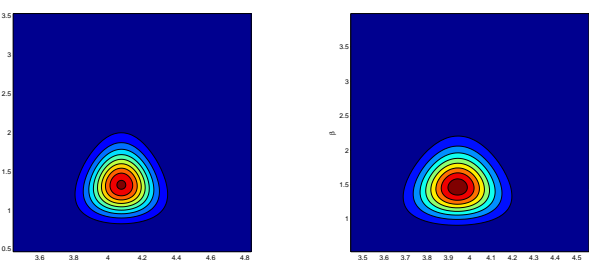

(h-4) $p\left(\mu, \beta \mid \mathcal{X}_{4, \text { pre }}\right)$

(h-5) $p\left(\mu, \beta \mid \mathcal{X}_{5, \text { pre }}\right)$

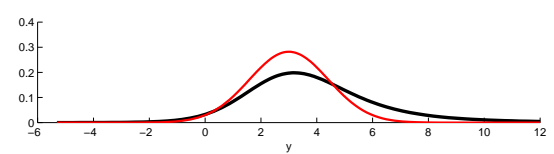

(i-1) $p\left(y \mid \mathcal{X}_{1, \text { pre }}, \mathcal{X}_{1, \text { post }}\right)$

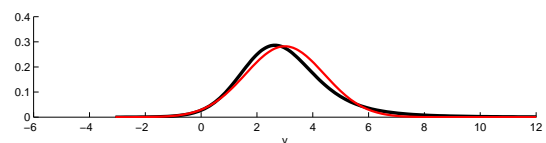

(i-4) $p\left(y \mid \mathcal{X}_{4, \text { pre }}, \mathcal{X}_{4, \text { post }}\right)$

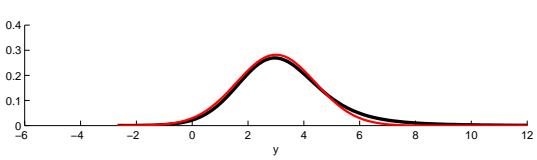

$(\mathrm{i}-3) p\left(y \mid \mathcal{X}_{3, \text { pre }}, \mathcal{X}_{3, \text { post }}\right)$

Figure 30S: Bayesian inferences on five datasets, each of which contains $n_{v}=5$ detect data out of $n=48$ samples. 


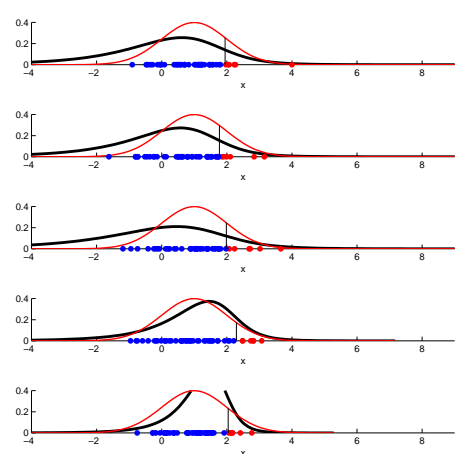

(a) $p\left(c_{\text {post }} \mid \mathcal{X}_{k, \text { post }}\right)$

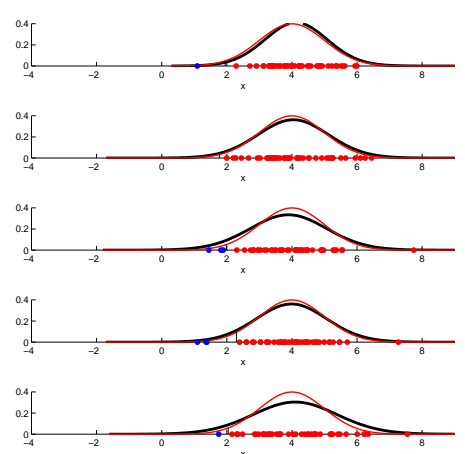

(d) $p\left(c_{\text {pre }} \mid \mathcal{X}_{k, \text { pre }}\right)$

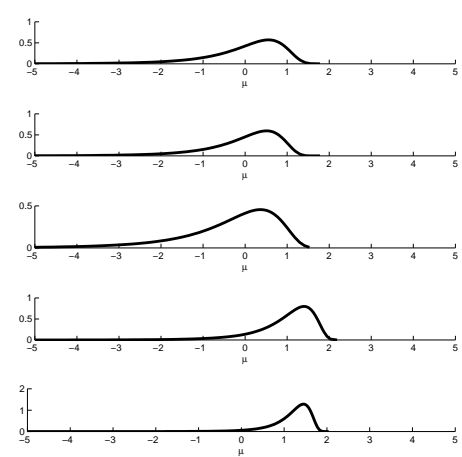

(b) $p\left(\mu_{1} \mid \mathcal{X}_{k, \text { post }}\right)$

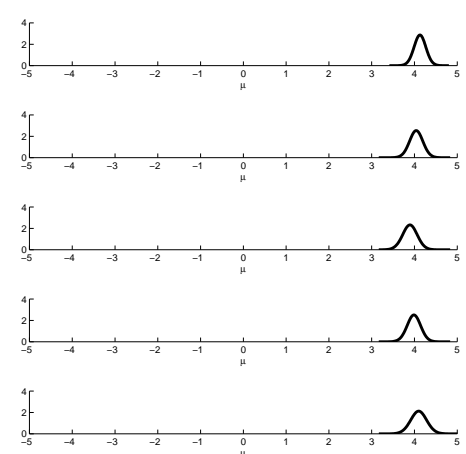

(e) $p\left(\mu_{1} \mid \mathcal{X}_{k, \text { pre }}\right)$

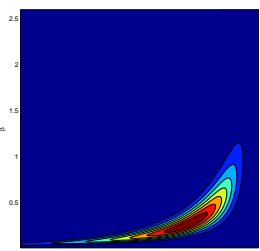

(g-3) $p\left(\mu, \beta \mid \mathcal{X}_{3, \text { post }}\right)$

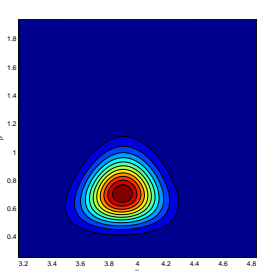

(h-3) $p\left(\mu, \beta \mid \mathcal{X}_{3, \text { pre }}\right)$

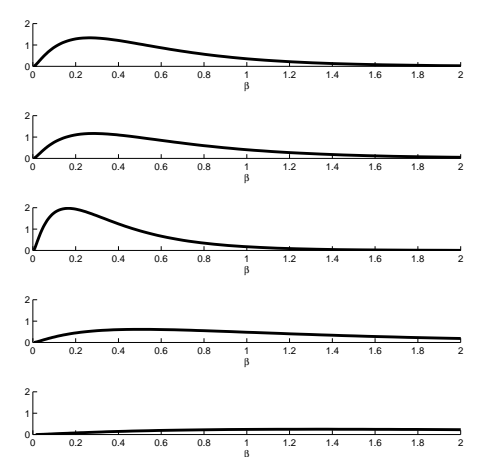

(c) $p\left(\beta_{1} \mid \mathcal{X}_{k, \text { post }}\right)$

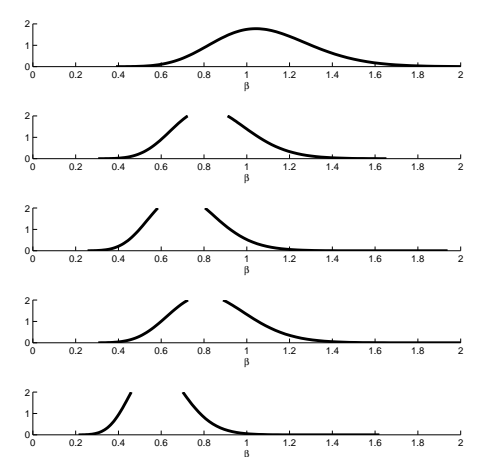

(f) $p\left(\beta_{1} \mid \mathcal{X}_{k, \text { pre }}\right)$

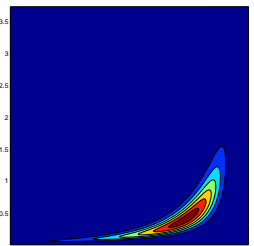

(g-1) $p\left(\mu, \beta \mid \mathcal{X}_{1, \text { post }}\right)$

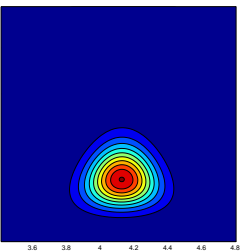

(h-1) $p\left(\mu, \beta \mid \mathcal{X}_{1, \text { pre }}\right)$

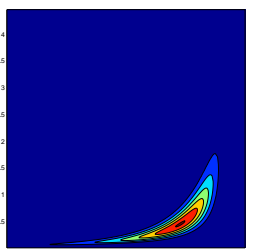

(g-2) $p\left(\mu, \beta \mid \mathcal{X}_{2, \text { post }}\right)$

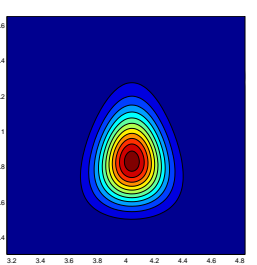

(h-2) $p\left(\mu, \beta \mid \mathcal{X}_{2, \text { pre }}\right)$

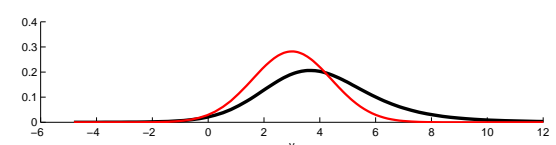

(i-2) $p\left(y \mid \mathcal{X}_{2, \text { pre }}, \mathcal{X}_{2, \text { post }}\right)$

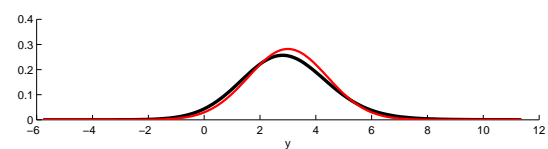

(i-5) $p\left(y \mid \mathcal{X}_{5, \text { pre }}, \mathcal{X}_{5, \text { post }}\right)$
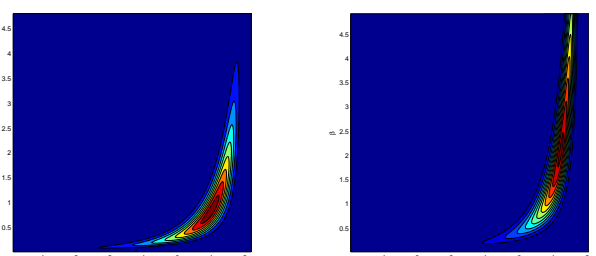

(g-4) $p\left(\mu, \beta \mid \mathcal{X}_{4, \text { post }}\right)$

$(\mathrm{g}-5) p\left(\mu, \beta \mid \mathcal{X}_{5, \text { post }}\right)$
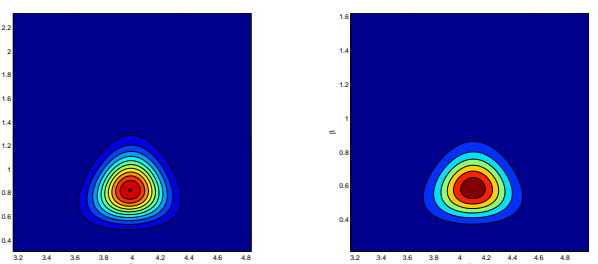

(h-4) $p\left(\mu, \beta \mid \mathcal{X}_{4, \text { pre }}\right)$

(h-5) $p\left(\mu, \beta \mid \mathcal{X}_{5, \text { pre }}\right)$

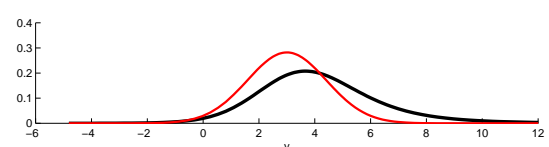

(i-1) $p\left(y \mid \mathcal{X}_{1, \text { pre }}, \mathcal{X}_{1, \text { post }}\right)$

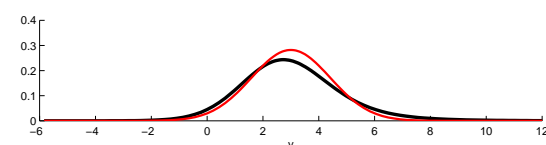

(i-4) $p\left(y \mid \mathcal{X}_{4, \text { pre }}, \mathcal{X}_{4, \text { post }}\right)$

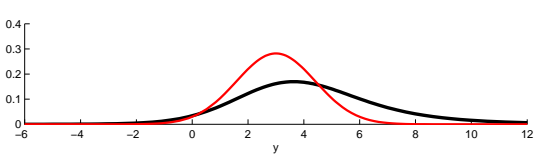

(i-3) $p\left(y \mid \mathcal{X}_{3, \text { pre }}, \mathcal{X}_{3, \text { post }}\right)$

Figure 31S: Bayesian inferences on five datasets, each of which contains $n_{v}=6$ detect data out of $n=48$ samples. 


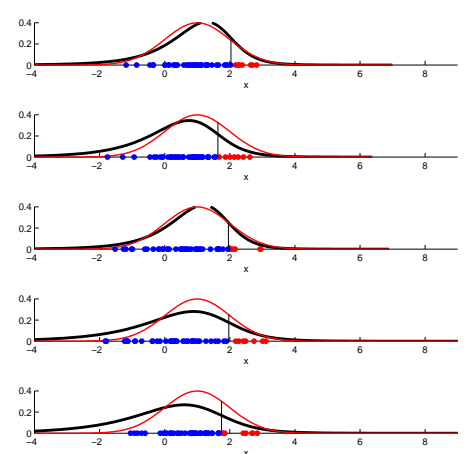

(a) $p\left(c_{\text {post }} \mid \mathcal{X}_{k, \text { post }}\right)$

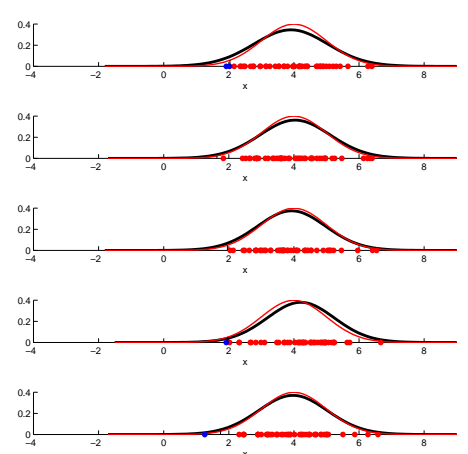

(d) $p\left(c_{\text {pre }} \mid \mathcal{X}_{k, \text { pre }}\right)$

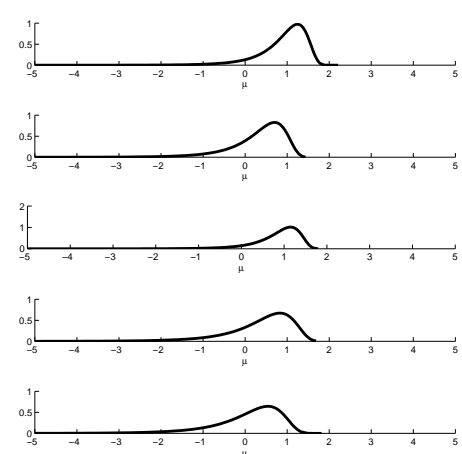

(b) $p\left(\mu_{1} \mid \mathcal{X}_{k, \text { post }}\right)$

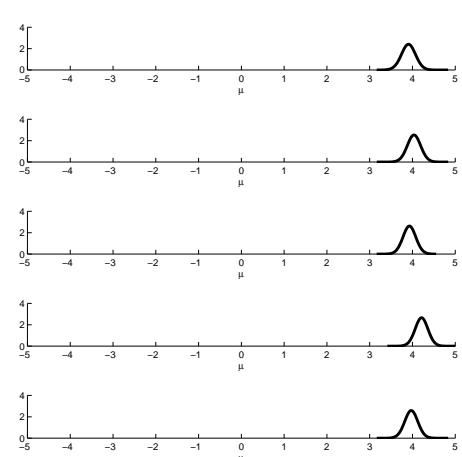

(e) $p\left(\mu_{1} \mid \mathcal{X}_{k, \text { pre }}\right)$

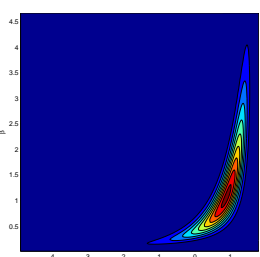

(g-3) $p\left(\mu, \beta \mid \mathcal{X}_{3, \text { post }}\right)$

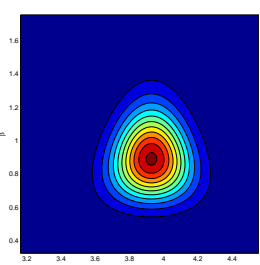

(h-3) $p\left(\mu, \beta \mid \mathcal{X}_{3, \text { pre }}\right)$

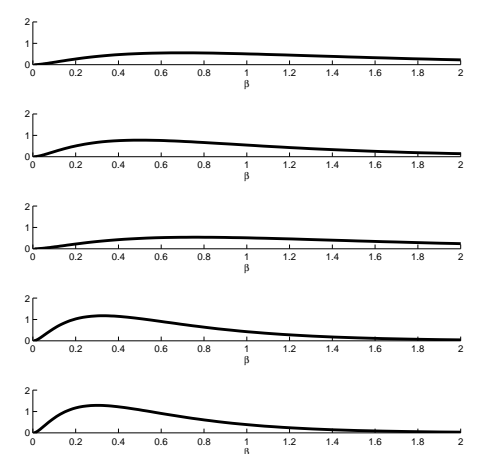

(c) $p\left(\beta_{1} \mid \mathcal{X}_{k, \text { post }}\right)$

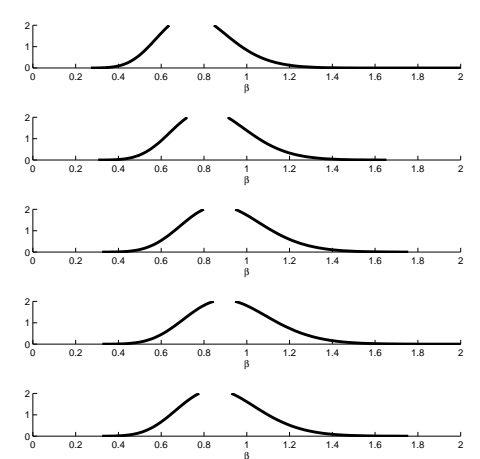

(f) $p\left(\beta_{1} \mid \mathcal{X}_{k, \text { pre }}\right)$

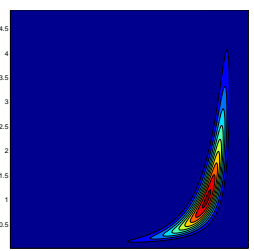

(g-1) $p\left(\mu, \beta \mid \mathcal{X}_{1, \text { post }}\right)$

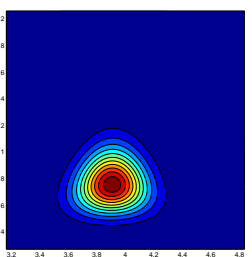

(h-1) $p\left(\mu, \beta \mid \mathcal{X}_{1, \text { pre }}\right)$

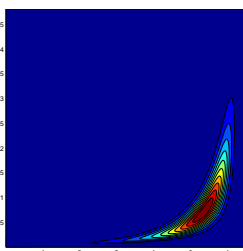

(g-2) $p\left(\mu, \beta \mid \mathcal{X}_{2, \text { post }}\right)$

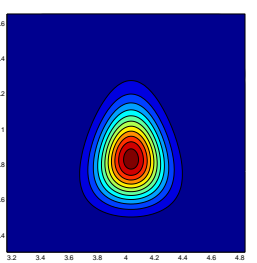

(h-2) $p\left(\mu, \beta \mid \mathcal{X}_{2, \text { pre }}\right)$

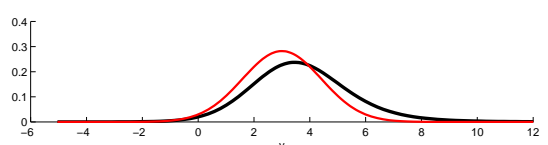

(i-2) $p\left(y \mid \mathcal{X}_{2, \text { pre }}, \mathcal{X}_{2, \text { post }}\right)$

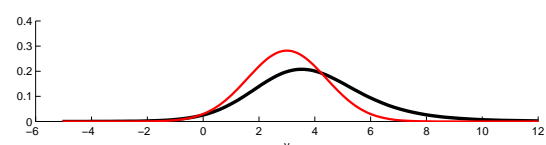

(i-5) $p\left(y \mid \mathcal{X}_{5, \text { pre }}, \mathcal{X}_{5, \text { post }}\right)$

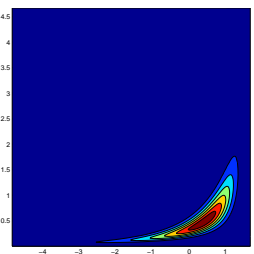

(g-4) $p\left(\mu, \beta \mid \mathcal{X}_{4, \text { post }}\right)$

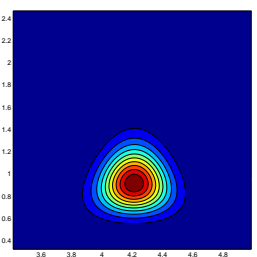

(h-4) $p\left(\mu, \beta \mid \mathcal{X}_{4, \text { pre }}\right)$

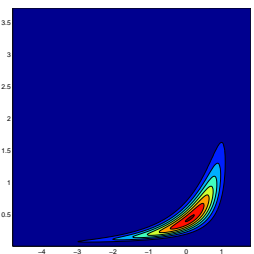

(g-5) $p\left(\mu, \beta \mid \mathcal{X}_{5, \text { post }}\right)$

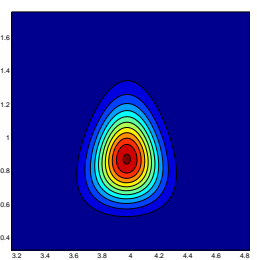

(h-5) $p\left(\mu, \beta \mid \mathcal{X}_{5, \text { pre }}\right)$

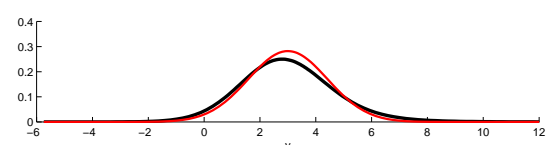

(i-1) $p\left(y \mid \mathcal{X}_{1, \text { pre }}, \mathcal{X}_{1, \text { post }}\right)$

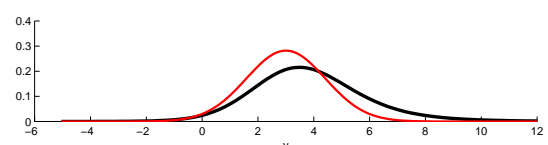

(i-4) $p\left(y \mid \mathcal{X}_{4, \text { pre }}, \mathcal{X}_{4, \text { post }}\right)$

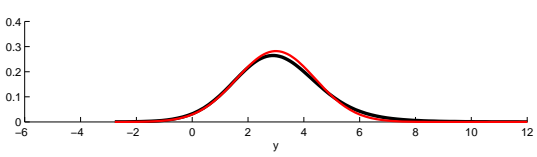

(i-3) $p\left(y \mid \mathcal{X}_{3, \text { pre }}, \mathcal{X}_{3, \text { post }}\right)$

Figure 32S: Bayesian inferences on five datasets, each of which contains $n_{v}=7$ detect data out of $n=48$ samples. 


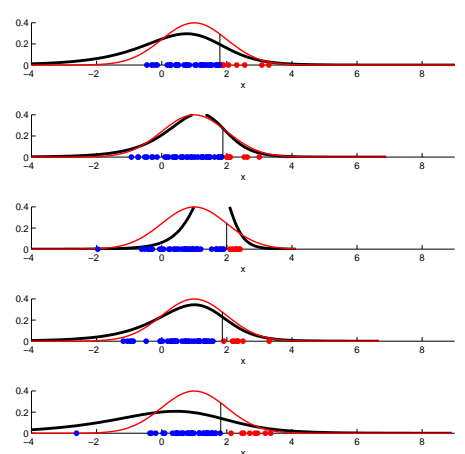

(a) $p\left(c_{\text {post }} \mid \mathcal{X}_{k, \text { post }}\right)$

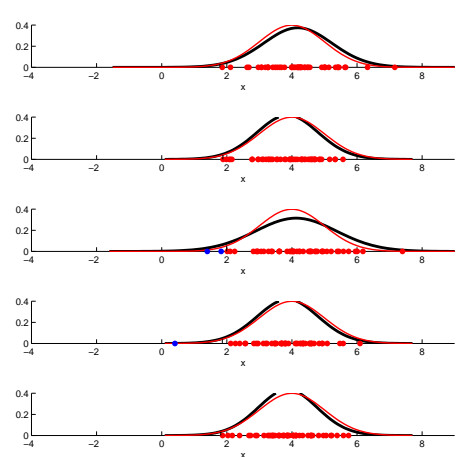

(d) $p\left(c_{\text {pre }} \mid \mathcal{X}_{k, \text { pre }}\right)$

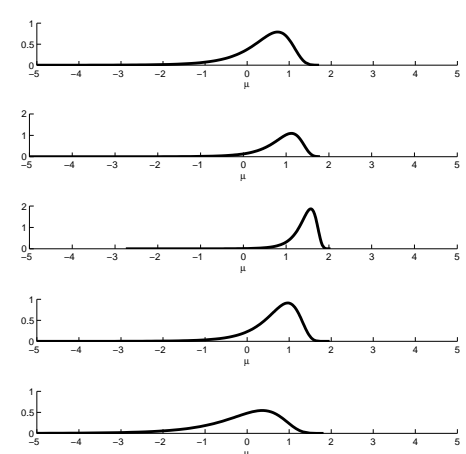

(b) $p\left(\mu_{1} \mid \mathcal{X}_{k, \text { post }}\right)$

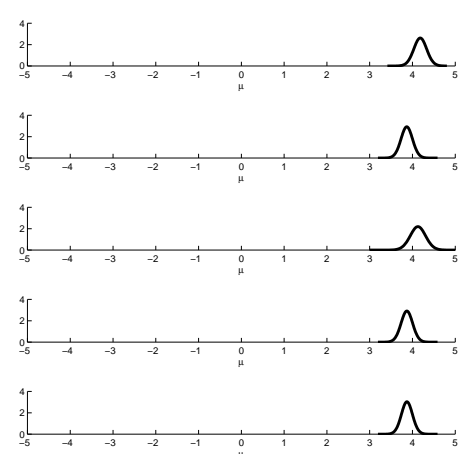

(e) $p\left(\mu_{1} \mid \mathcal{X}_{k, \text { pre }}\right)$

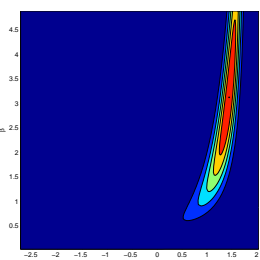

(g-3) $p\left(\mu, \beta \mid \mathcal{X}_{3, \text { post }}\right)$

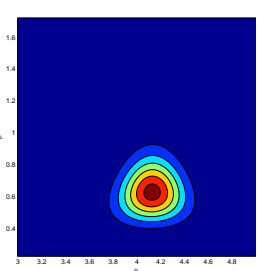

(h-3) $p\left(\mu, \beta \mid \mathcal{X}_{3, \text { pre }}\right)$

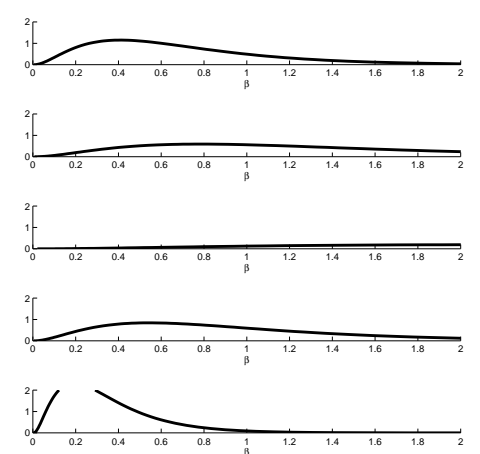

(c) $p\left(\beta_{1} \mid \mathcal{X}_{k, \text { post }}\right)$

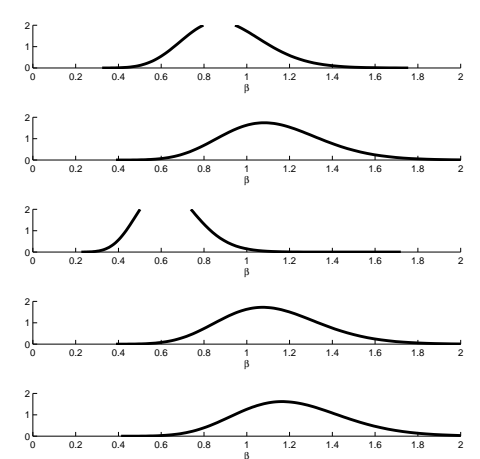

(f) $p\left(\beta_{1} \mid \mathcal{X}_{k, \text { pre }}\right)$

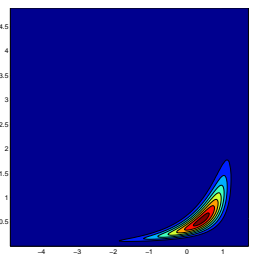

(g-1) $p\left(\mu, \beta \mid \mathcal{X}_{1, \text { post }}\right)$

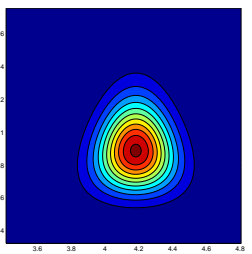

(h-1) $p\left(\mu, \beta \mid \mathcal{X}_{1, \text { pre }}\right)$

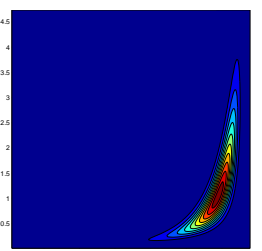

$(\mathrm{g}-2) p\left(\mu, \beta \mid \mathcal{X}_{2, \text { post }}\right)$

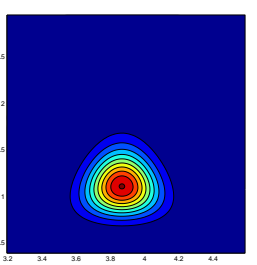

(h-2) $p\left(\mu, \beta \mid \mathcal{X}_{2, \text { pre }}\right)$

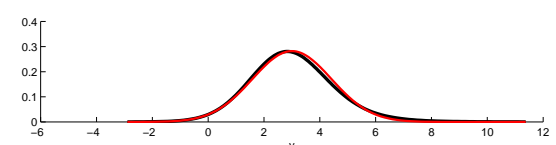

(i-2) $p\left(y \mid \mathcal{X}_{2, \text { pre }}, \mathcal{X}_{2, \text { post }}\right)$

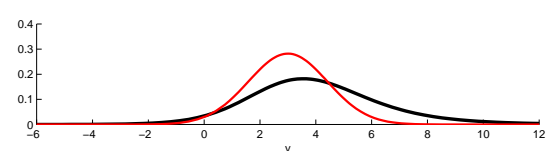

(i-5) $p\left(y \mid \mathcal{X}_{5, \text { pre }}, \mathcal{X}_{5, \text { post }}\right)$

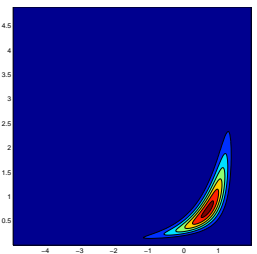

(g-4) $p\left(\mu, \beta \mid \mathcal{X}_{4, \text { post }}\right)$

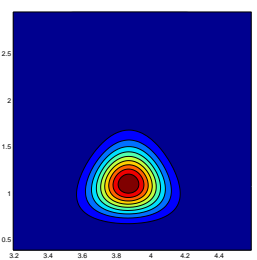

(h-4) $p\left(\mu, \beta \mid \mathcal{X}_{4, \text { pre }}\right)$

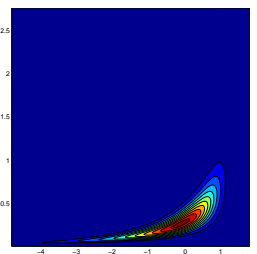

(g-5) $p\left(\mu, \beta \mid \mathcal{X}_{5, \text { post }}\right)$

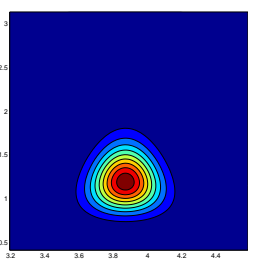

(h-5) $p\left(\mu, \beta \mid \mathcal{X}_{5, \text { pre }}\right)$

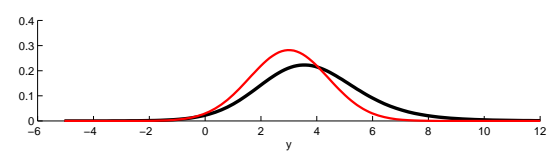

(i-1) $p\left(y \mid \mathcal{X}_{1, \text { pre }}, \mathcal{X}_{1, \text { post }}\right)$

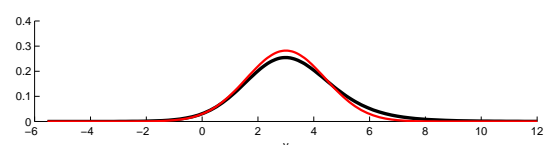

(i-4) $p\left(y \mid \mathcal{X}_{4, \text { pre }}, \mathcal{X}_{4, \text { post }}\right)$

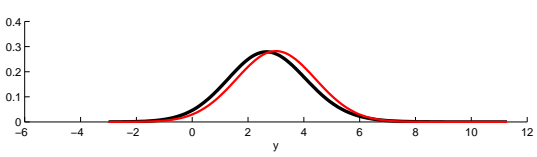

(i-3) $p\left(y \mid \mathcal{X}_{3, \text { pre }}, \mathcal{X}_{3, \text { post }}\right)$

Figure 33S: Bayesian inferences on five datasets, each of which contains $n_{v}=8$ detect data out of $n=48$ samples. 


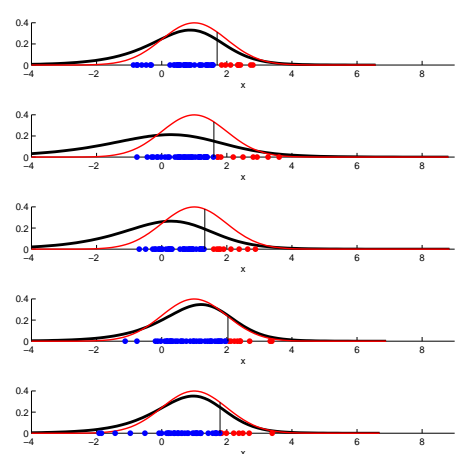

(a) $p\left(c_{\text {post }} \mid \mathcal{X}_{k, \text { post }}\right)$

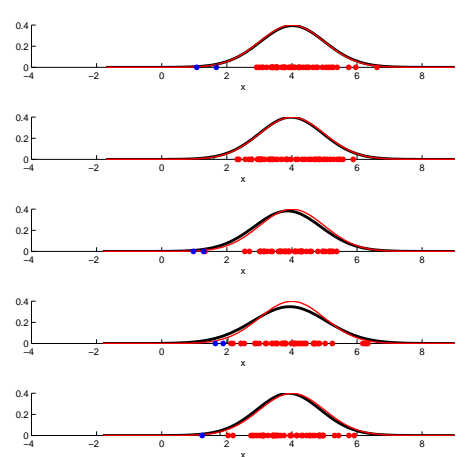

(d) $p\left(c_{\text {pre }} \mid \mathcal{X}_{k, \text { pre }}\right)$

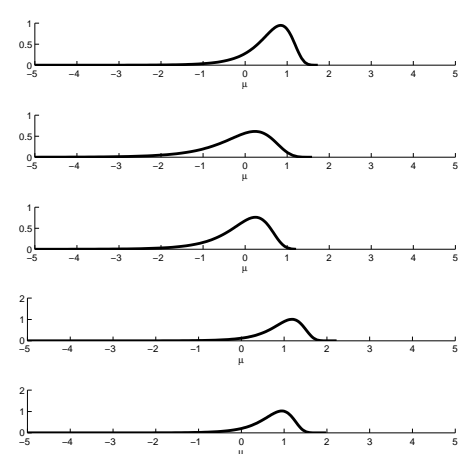

(b) $p\left(\mu_{1} \mid \mathcal{X}_{k, \text { post }}\right)$

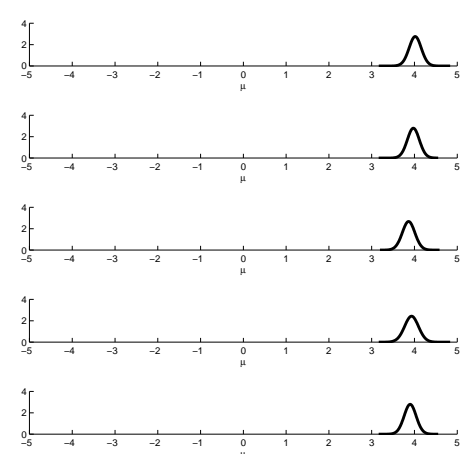

(e) $p\left(\mu_{1} \mid \mathcal{X}_{k, \text { pre }}\right)$

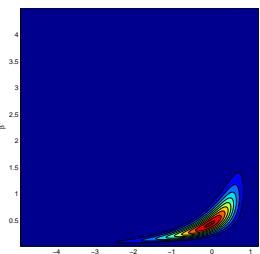

(g-3) $p\left(\mu, \beta \mid \mathcal{X}_{3, \text { post }}\right)$

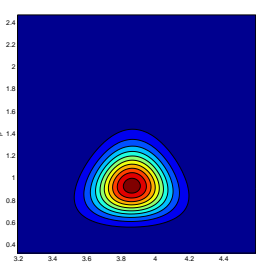

(h-3) $p\left(\mu, \beta \mid \mathcal{X}_{3, \text { pre }}\right)$

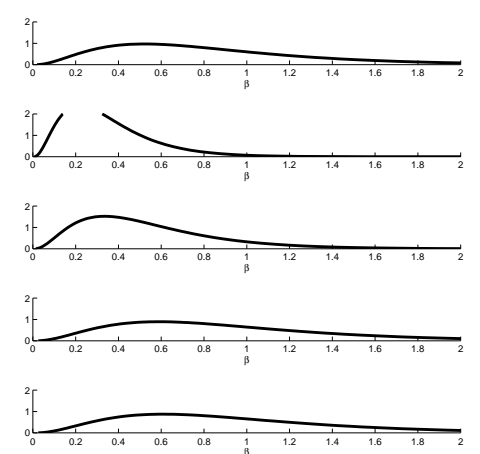

(c) $p\left(\beta_{1} \mid \mathcal{X}_{k, \text { post }}\right)$

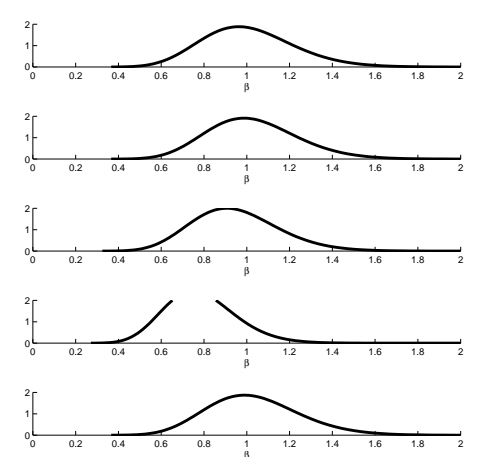

(f) $p\left(\beta_{1} \mid \mathcal{X}_{k, \text { pre }}\right)$

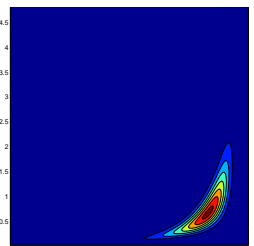

(g-1) $p\left(\mu, \beta \mid \mathcal{X}_{1, \text { post }}\right)$

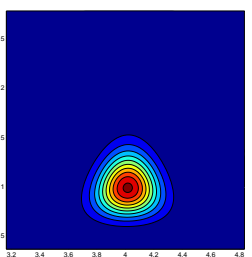

(h-1) $p\left(\mu, \beta \mid \mathcal{X}_{1, \text { pre }}\right)$

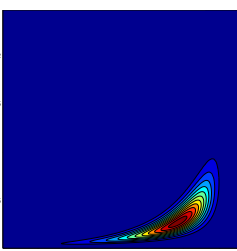

(g-2) $p\left(\mu, \beta \mid \mathcal{X}_{2, \text { post }}\right)$

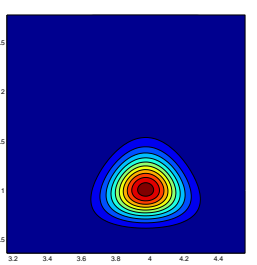

(h-2) $p\left(\mu, \beta \mid \mathcal{X}_{2, \text { pre }}\right)$

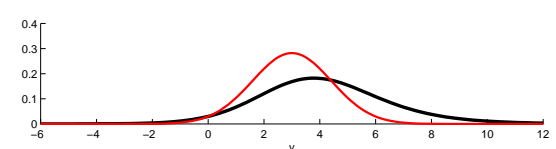

(i-2) $p\left(y \mid \mathcal{X}_{2, \text { pre }}, \mathcal{X}_{2, \text { post }}\right)$

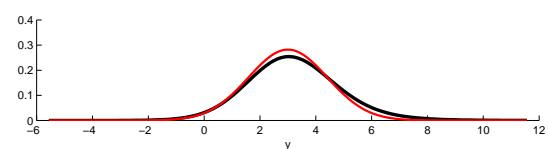

(i-5) $p\left(y \mid \mathcal{X}_{5, \text { pre }}, \mathcal{X}_{5, \text { post }}\right)$
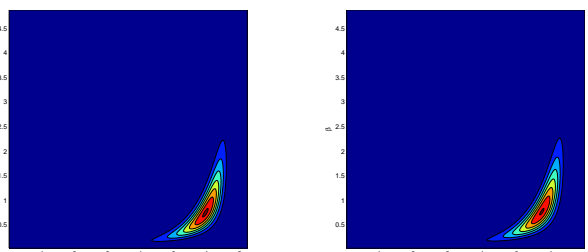

(g-4) $p\left(\mu, \beta \mid \mathcal{X}_{4, \text { post }}\right)$

(g-5) $p\left(\mu, \beta \mid \mathcal{X}_{5, \text { post }}\right)$
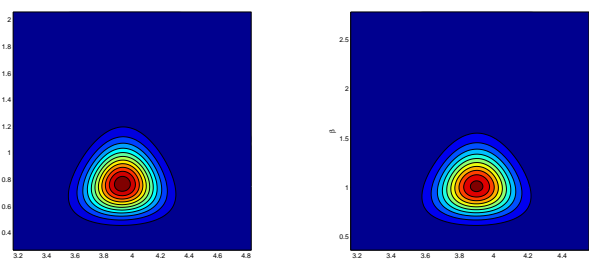

(h-4) $p\left(\mu, \beta \mid \mathcal{X}_{4, \text { pre }}\right)$

(h-5) $p\left(\mu, \beta \mid \mathcal{X}_{5, \text { pre }}\right)$

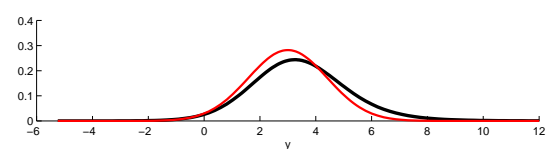

(i-1) $p\left(y \mid \mathcal{X}_{1, \text { pre }}, \mathcal{X}_{1, \text { post }}\right)$

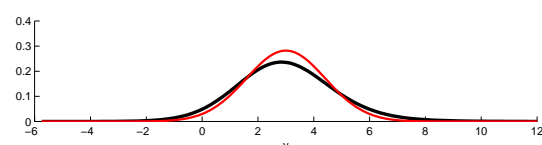

(i-4) $p\left(y \mid \mathcal{X}_{4, \text { pre }}, \mathcal{X}_{4, \text { post }}\right)$

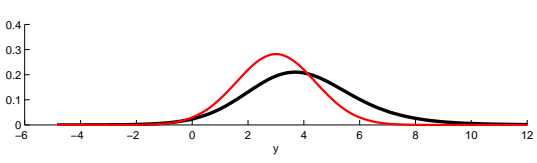

(i-3) $p\left(y \mid \mathcal{X}_{3, \text { pre }}, \mathcal{X}_{3, \text { post }}\right)$

Figure 34S: Bayesian inferences on five datasets, each of which contains $n_{v}=9$ detect data out of $n=48$ samples. 


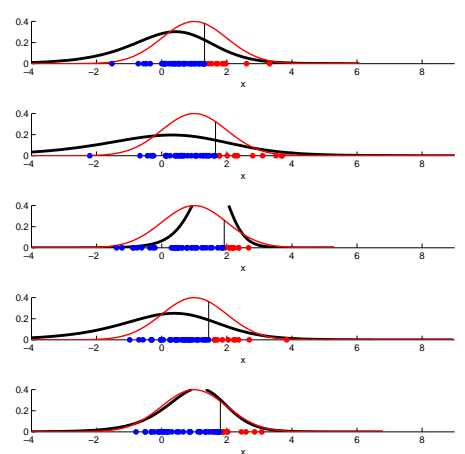

(a) $p\left(c_{\text {post }} \mid \mathcal{X}_{k, \text { post }}\right)$

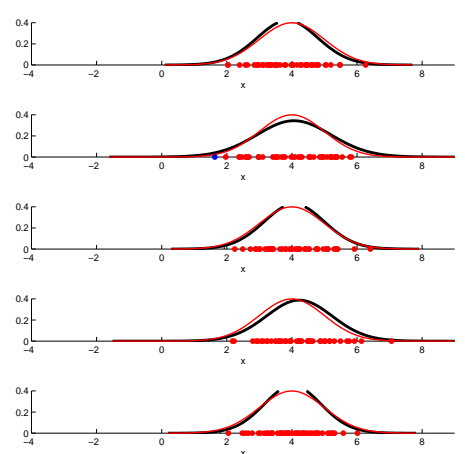

(d) $p\left(c_{\text {pre }} \mid \mathcal{X}_{k, \text { pre }}\right)$

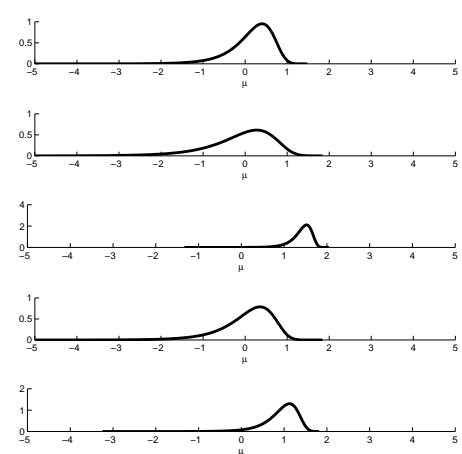

(b) $p\left(\mu_{1} \mid \mathcal{X}_{k, \text { post }}\right)$

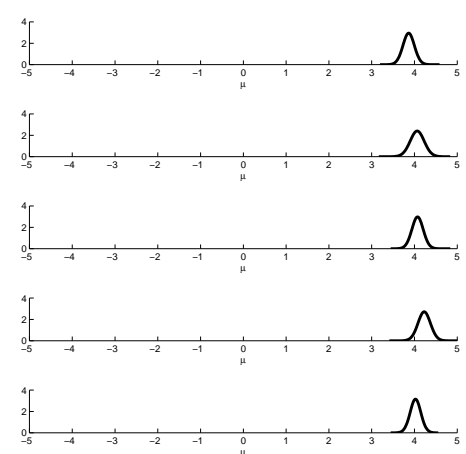

(e) $p\left(\mu_{1} \mid \mathcal{X}_{k, \text { pre }}\right)$

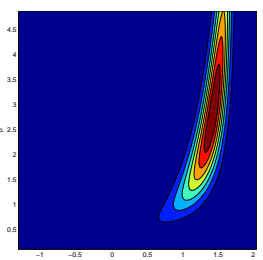

(g-3) $p\left(\mu, \beta \mid \mathcal{X}_{3, \text { post }}\right)$

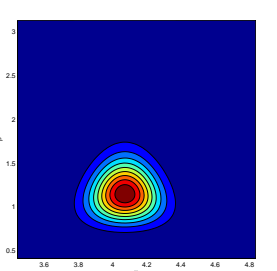

(h-3) $p\left(\mu, \beta \mid \mathcal{X}_{3, \text { pre }}\right)$

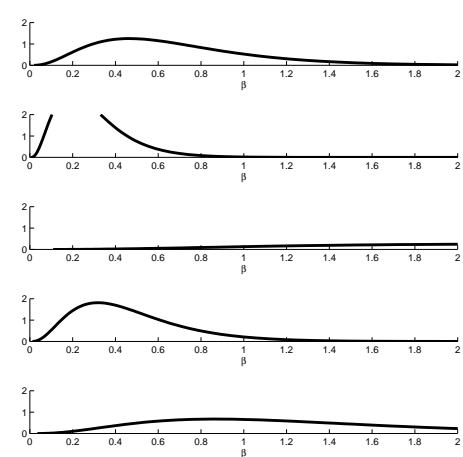

(c) $p\left(\beta_{1} \mid \mathcal{X}_{k, \text { post }}\right)$

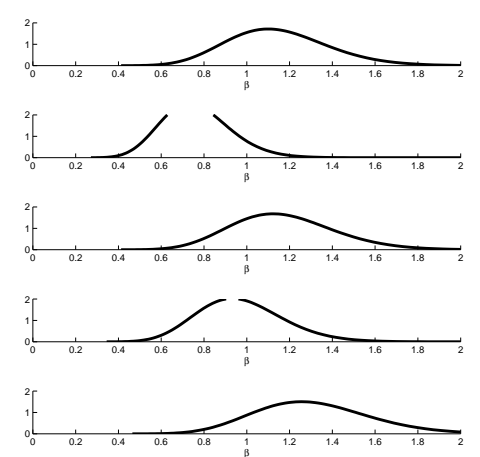

(f) $p\left(\beta_{1} \mid \mathcal{X}_{k, \text { pre }}\right)$

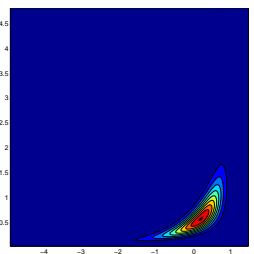

(g-1) $p\left(\mu, \beta \mid \mathcal{X}_{1, \text { post }}\right)$

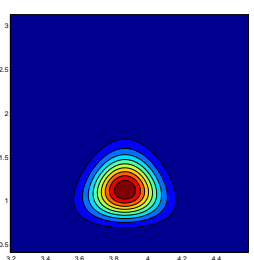

(h-1) $p\left(\mu, \beta \mid \mathcal{X}_{1, \text { pre }}\right)$

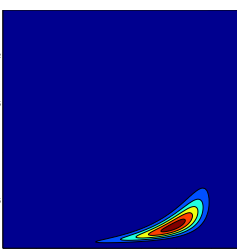

(g-2) $p\left(\mu, \beta \mid \mathcal{X}_{2, \text { post }}\right)$

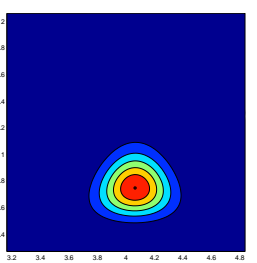

(h-2) $p\left(\mu, \beta \mid \mathcal{X}_{2, \text { pre }}\right)$

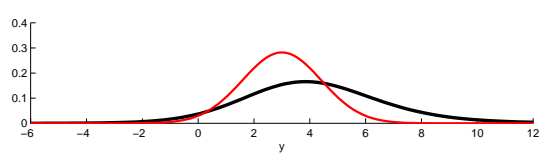

(i-2) $p\left(y \mid \mathcal{X}_{2, \text { pre }}, \mathcal{X}_{2, \text { post }}\right)$

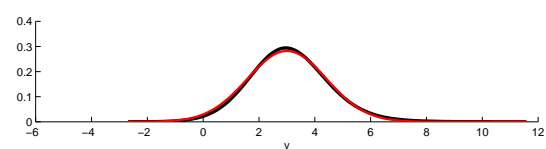

(i-5) $p\left(y \mid \mathcal{X}_{5, \text { pre }}, \mathcal{X}_{5, \text { post }}\right)$

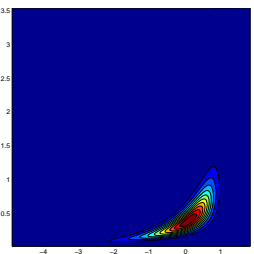

(g-4) $p\left(\mu, \beta \mid \mathcal{X}_{4, \text { post }}\right)$

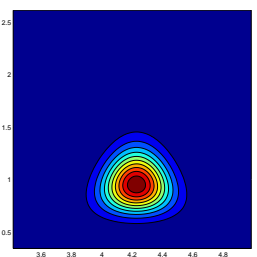

(h-4) $p\left(\mu, \beta \mid \mathcal{X}_{4, \text { pre }}\right)$

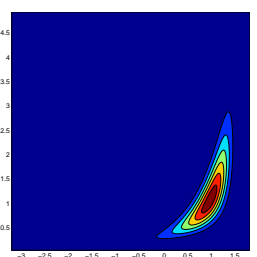

(g-5) $p\left(\mu, \beta \mid \mathcal{X}_{5, \text { post }}\right)$

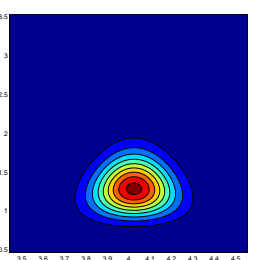

(h-5) $p\left(\mu, \beta \mid \mathcal{X}_{5, \text { pre }}\right)$

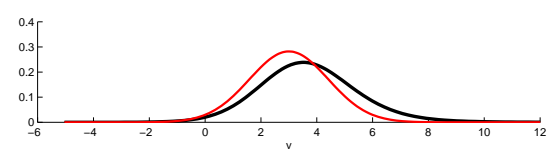

(i-1) $p\left(y \mid \mathcal{X}_{1, \text { pre }}, \mathcal{X}_{1, \text { post }}\right)$

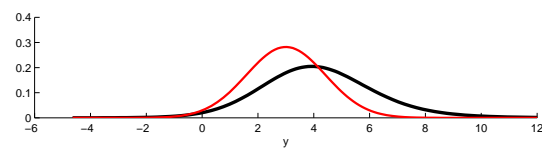

(i-4) $p\left(y \mid \mathcal{X}_{4, \text { pre }}, \mathcal{X}_{4, \text { post }}\right)$

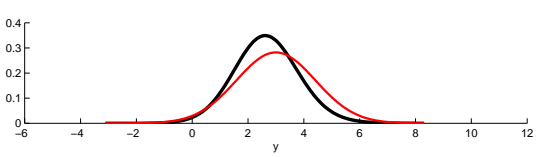

(i-3) $p\left(y \mid \mathcal{X}_{3, \text { pre }}, \mathcal{X}_{3, \text { post }}\right)$

Figure 35S: Bayesian inferences on five datasets, each of which contains $n_{v}=10$ detect data out of $n=48$ samples. 


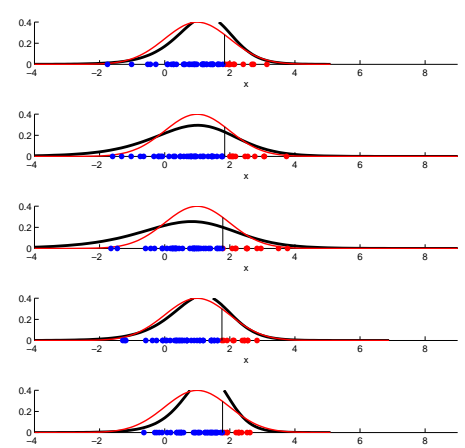

(a) $p\left(c_{\text {post }} \mid \mathcal{X}_{k, \text { post }}\right)$

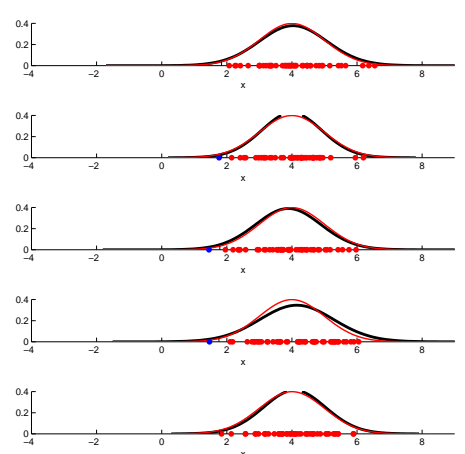

(d) $p\left(c_{\text {pre }} \mid \mathcal{X}_{k, \text { pre }}\right)$

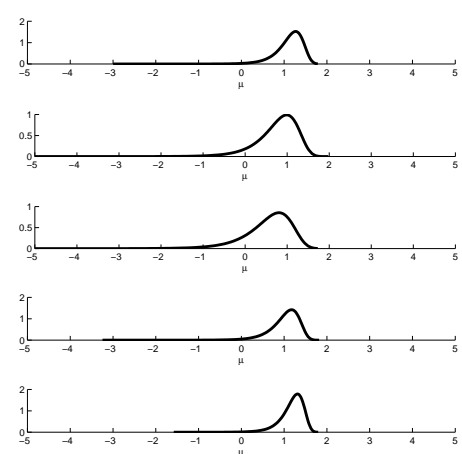

(b) $p\left(\mu_{1} \mid \mathcal{X}_{k, \text { post }}\right)$

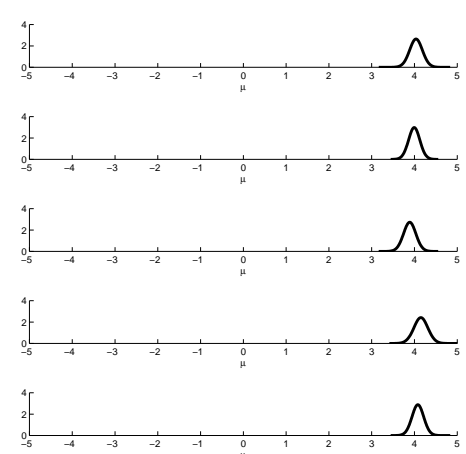

(e) $p\left(\mu_{1} \mid \mathcal{X}_{k, \text { pre }}\right)$

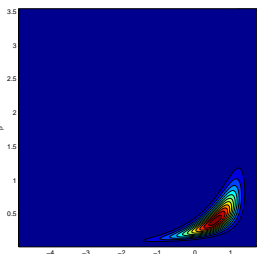

(g-3) $p\left(\mu, \beta \mid \mathcal{X}_{3, \text { post }}\right)$

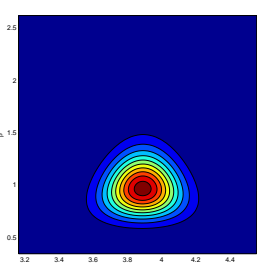

(h-3) $p\left(\mu, \beta \mid \mathcal{X}_{3, \text { pre }}\right)$

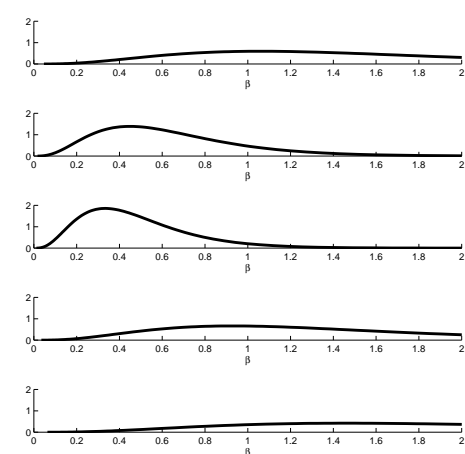

(c) $p\left(\beta_{1} \mid \mathcal{X}_{k, \text { post }}\right)$

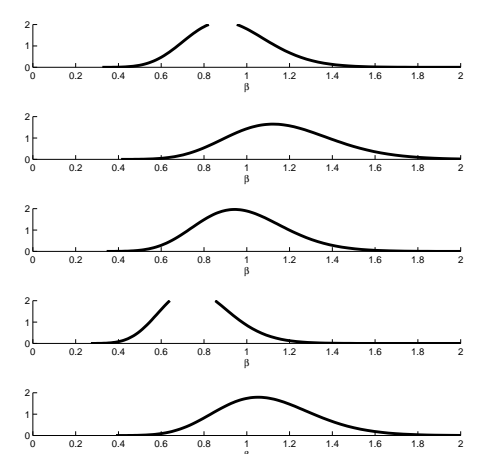

(f) $p\left(\beta_{1} \mid \mathcal{X}_{k, \text { pre }}\right)$
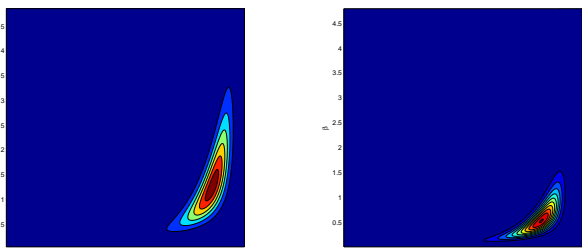

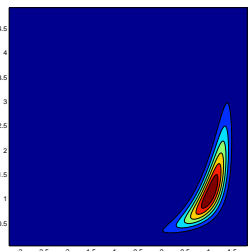

(g-4) $p\left(\mu, \beta \mid \mathcal{X}_{4, \text { post }}\right)$

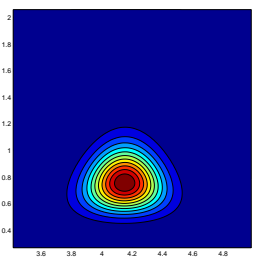

(h-4) $p\left(\mu, \beta \mid \mathcal{X}_{4, \text { pre }}\right)$

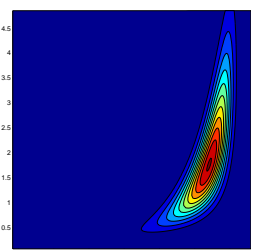

(g-5) $p\left(\mu, \beta \mid \mathcal{X}_{5, \text { post }}\right)$

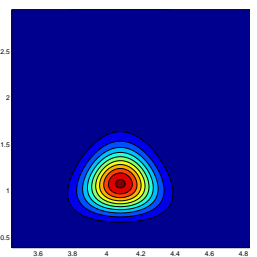

(h-5) $p\left(\mu, \beta \mid \mathcal{X}_{5, \text { pre }}\right)$

(h-1) $p\left(\mu, \beta \mid \mathcal{X}_{1, \text { pre }}\right)$

(h-2) $p\left(\mu, \beta \mid \mathcal{X}_{2, \text { pre }}\right)$

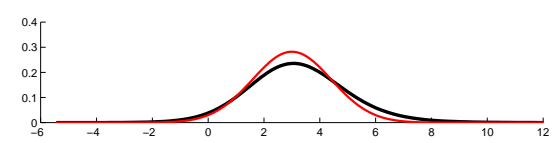

(i-2) $p\left(y \mid \mathcal{X}_{2, \text { pre }}, \mathcal{X}_{2, \text { post }}\right)$

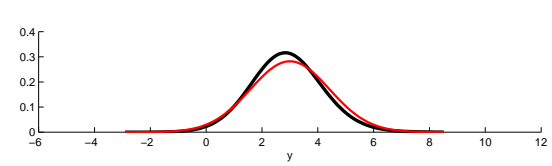

(i-5) $p\left(y \mid \mathcal{X}_{5, \text { pre }}, \mathcal{X}_{5, \text { post }}\right)$

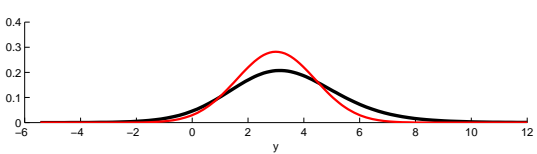

(i-3) $p\left(y \mid \mathcal{X}_{3, \text { pre }}, \mathcal{X}_{3, \text { post }}\right)$

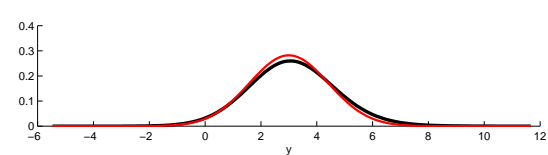

(i-4) $p\left(y \mid \mathcal{X}_{4, \text { pre }}, \mathcal{X}_{4, \text { post }}\right)$

Figure 36S: Bayesian inferences on five datasets, each of which contains $n_{v}=11$ detect data out of $n=48$ samples. 


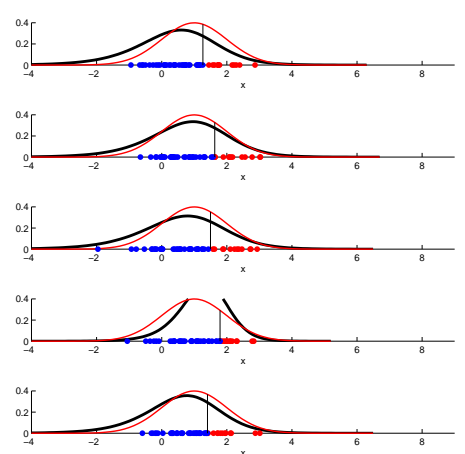

(a) $p\left(c_{\text {post }} \mid \mathcal{X}_{k, \text { post }}\right)$

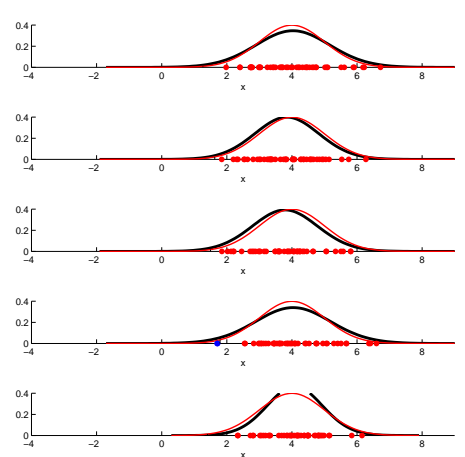

(d) $p\left(c_{\text {pre }} \mid \mathcal{X}_{k, \text { pre }}\right)$

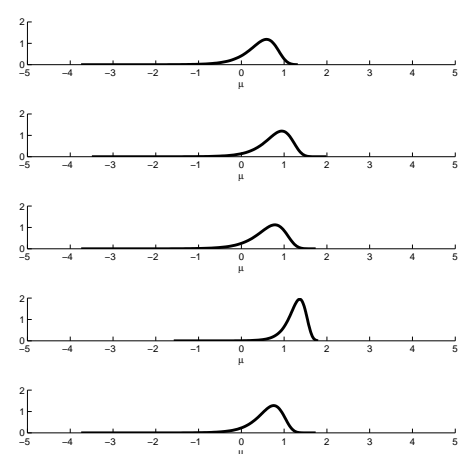

(b) $p\left(\mu_{1} \mid \mathcal{X}_{k, \text { post }}\right)$

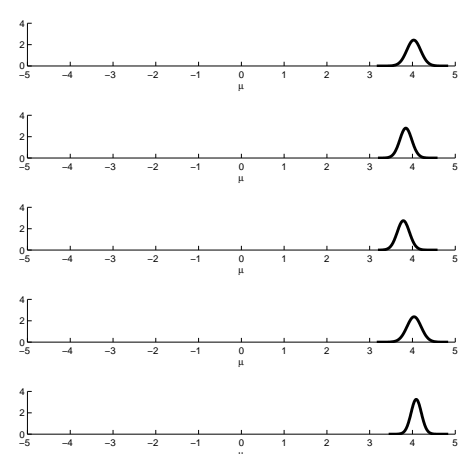

(e) $p\left(\mu_{1} \mid \mathcal{X}_{k, \text { pre }}\right)$

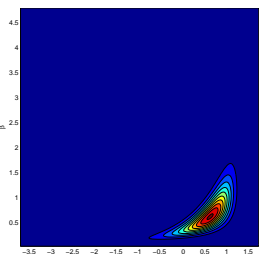

(g-3) $p\left(\mu, \beta \mid \mathcal{X}_{3, \text { post }}\right)$

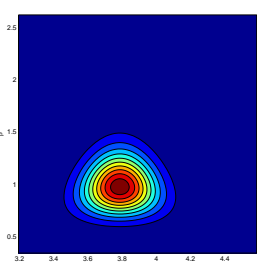

(h-3) $p\left(\mu, \beta \mid \mathcal{X}_{3, \text { pre }}\right)$

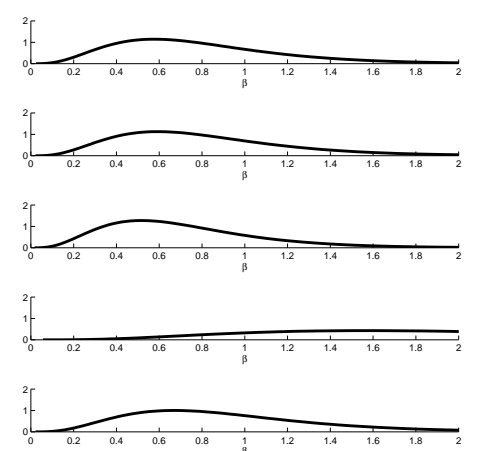

(c) $p\left(\beta_{1} \mid \mathcal{X}_{k, \text { post }}\right)$

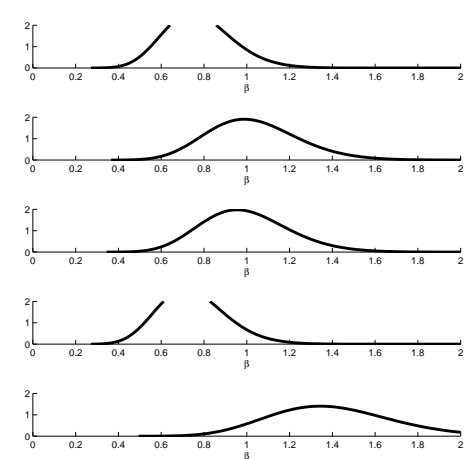

(f) $p\left(\beta_{1} \mid \mathcal{X}_{k, \text { pre }}\right)$

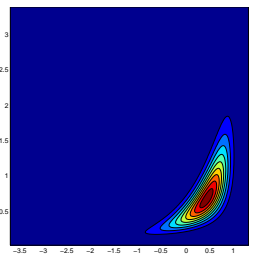

(g-1) $p\left(\mu, \beta \mid \mathcal{X}_{1, \text { post }}\right)$

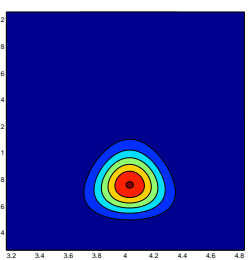

(h-1) $p\left(\mu, \beta \mid \mathcal{X}_{1, \text { pre }}\right)$

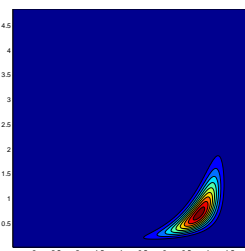

(g-2) $p\left(\mu, \beta \mid \mathcal{X}_{2, \text { post }}\right)$

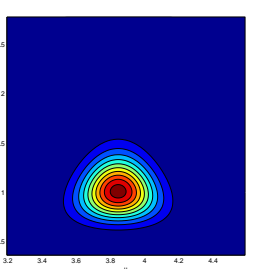

(h-2) $p\left(\mu, \beta \mid \mathcal{X}_{2, \text { pre }}\right)$

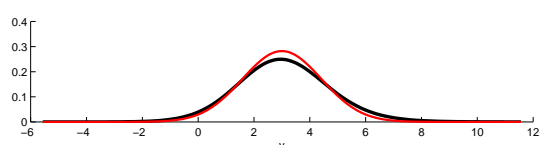

(i-2) $p\left(y \mid \mathcal{X}_{2, \text { pre }}, \mathcal{X}_{2, \text { post }}\right)$

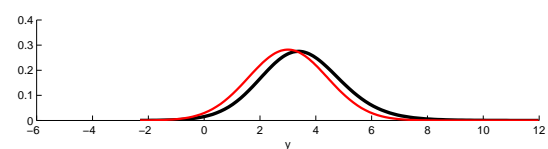

(i-5) $p\left(y \mid \mathcal{X}_{5, \text { pre }}, \mathcal{X}_{5, \text { post }}\right)$

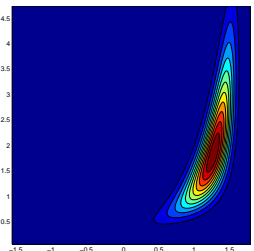

(g-4) $p\left(\mu, \beta \mid \mathcal{X}_{4, \text { post }}\right)$

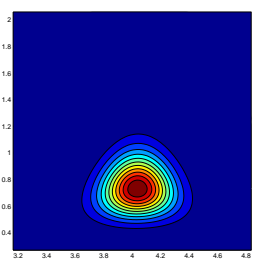

(h-4) $p\left(\mu, \beta \mid \mathcal{X}_{4, \text { pre }}\right)$

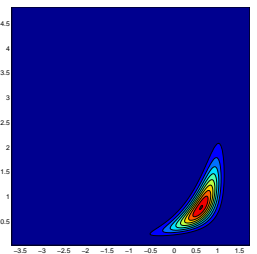

(g-5) $p\left(\mu, \beta \mid \mathcal{X}_{5, \text { post }}\right)$

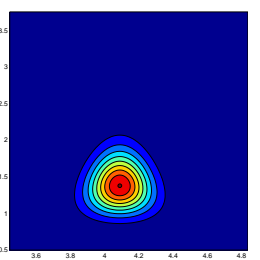

(h-5) $p\left(\mu, \beta \mid \mathcal{X}_{5, \text { pre }}\right)$

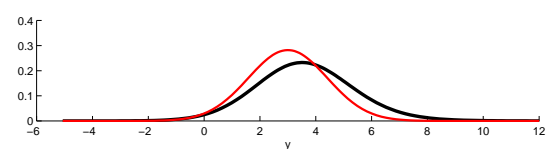

(i-1) $p\left(y \mid \mathcal{X}_{1, \text { pre }}, \mathcal{X}_{1, \text { post }}\right)$

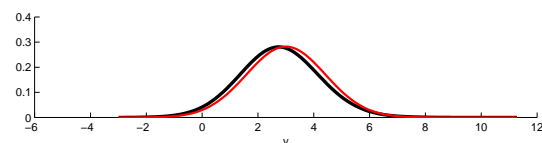

(i-4) $p\left(y \mid \mathcal{X}_{4, \text { pre }}, \mathcal{X}_{4, \text { post }}\right)$

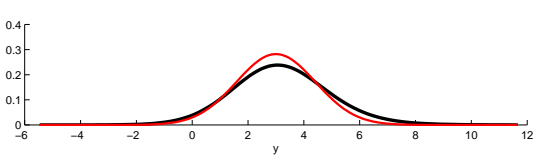

(i-3) $p\left(y \mid \mathcal{X}_{3, \text { pre }}, \mathcal{X}_{3, \text { post }}\right)$

Figure 37S: Bayesian inferences on five datasets, each of which contains $n_{v}=12$ detect data out of $n=48$ samples. 


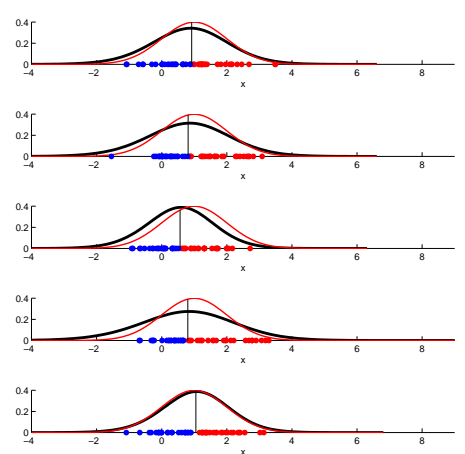

(a) $p\left(c_{\text {post }} \mid \mathcal{X}_{k, \text { post }}\right)$

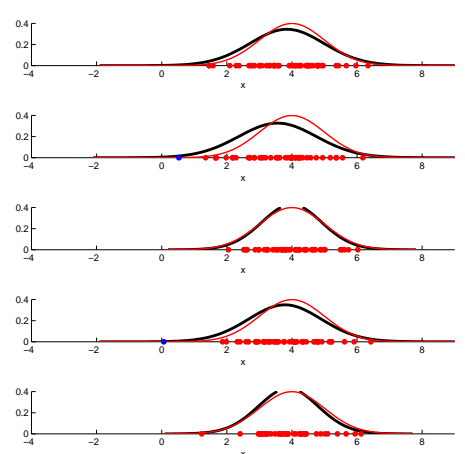

(d) $p\left(c_{\text {pre }} \mid \mathcal{X}_{k, \text { pre }}\right)$

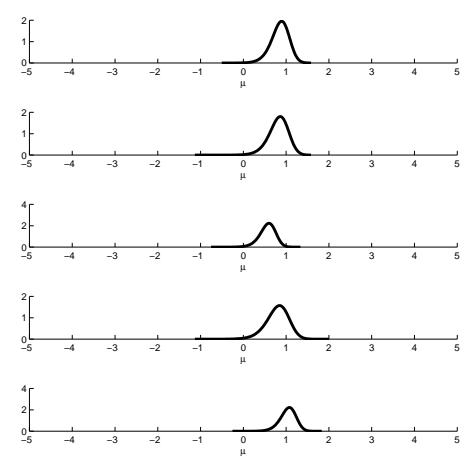

(b) $p\left(\mu_{1} \mid \mathcal{X}_{k, \text { post }}\right)$

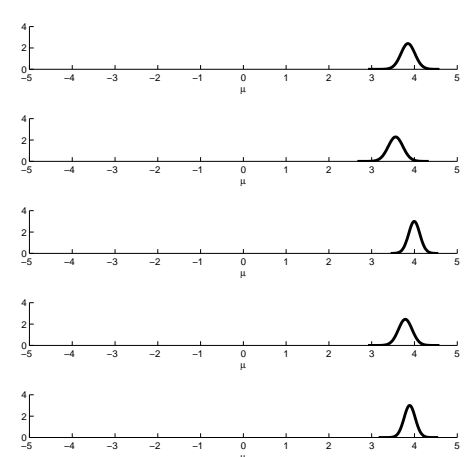

(e) $p\left(\mu_{1} \mid \mathcal{X}_{k, \text { pre }}\right)$

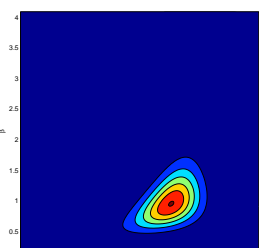

(g-3) $p\left(\mu, \beta \mid \mathcal{X}_{3, \text { post }}\right)$

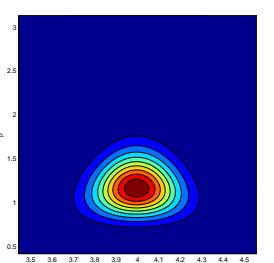

(h-3) $p\left(\mu, \beta \mid \mathcal{X}_{3, \text { pre }}\right)$

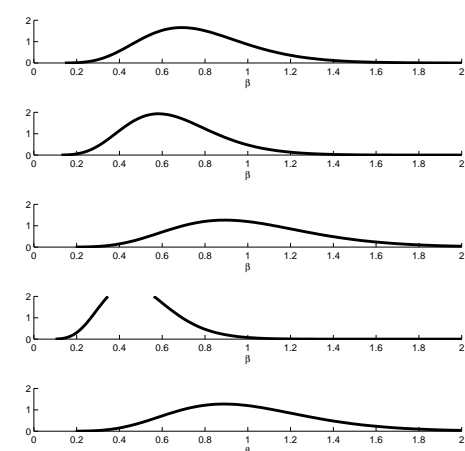

(c) $p\left(\beta_{1} \mid \mathcal{X}_{k, \text { post }}\right)$

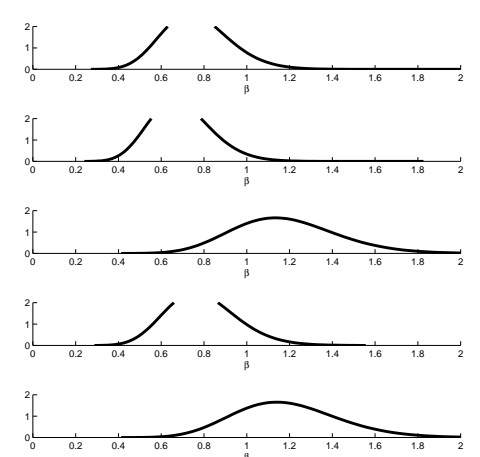

(f) $p\left(\beta_{1} \mid \mathcal{X}_{k, \text { pre }}\right)$

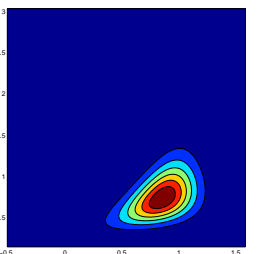

(g-1) $p\left(\mu, \beta \mid \mathcal{X}_{1, \text { post }}\right)$

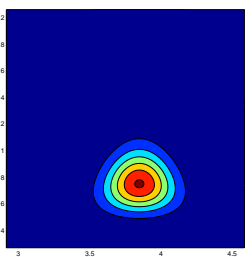

(h-1) $p\left(\mu, \beta \mid \mathcal{X}_{1, \text { pre }}\right)$

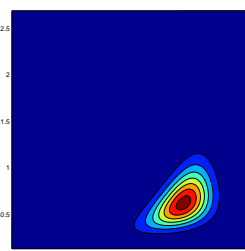

(g-2) $p\left(\mu, \beta \mid \mathcal{X}_{2, \text { post }}\right)$

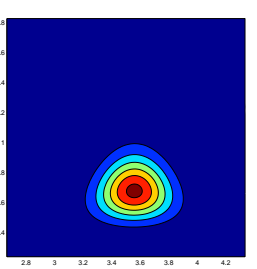

(h-2) $p\left(\mu, \beta \mid \mathcal{X}_{2, \text { pre }}\right)$

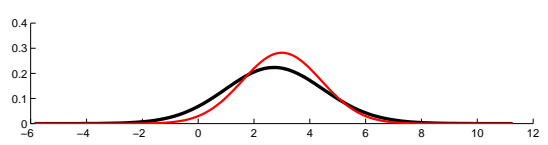

(i-2) $p\left(y \mid \mathcal{X}_{2, \text { pre }}, \mathcal{X}_{2, \text { post }}\right)$

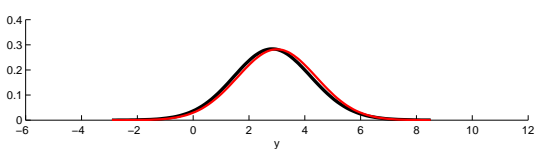

(i-5) $p\left(y \mid \mathcal{X}_{5, \text { pre }}, \mathcal{X}_{5, \text { post }}\right)$

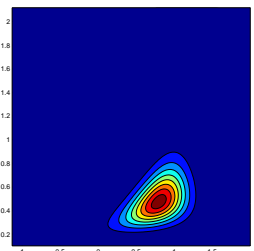

(g-4) $p\left(\mu, \beta \mid \mathcal{X}_{4, \text { post }}\right)$

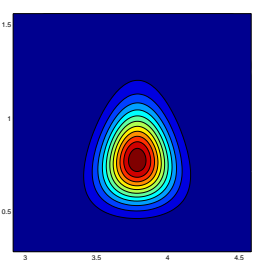

(h-4) $p\left(\mu, \beta \mid \mathcal{X}_{4, \text { pre }}\right)$

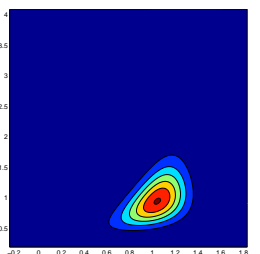

(g-5) $p\left(\mu, \beta \mid \mathcal{X}_{5, \text { post }}\right)$

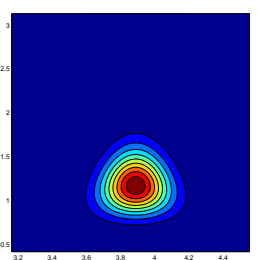

(h-5) $p\left(\mu, \beta \mid \mathcal{X}_{5, \text { pre }}\right)$

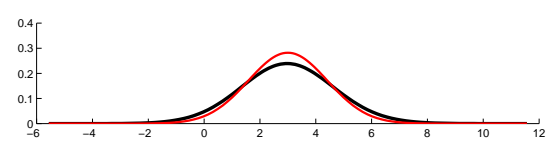

(i-1) $p\left(y \mid \mathcal{X}_{1, \text { pre }}, \mathcal{X}_{1, \text { post }}\right)$

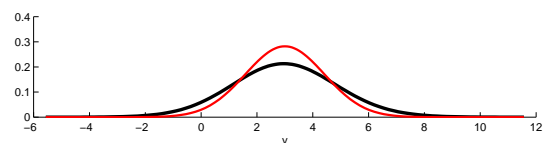

(i-4) $p\left(y \mid \mathcal{X}_{4, \text { pre }}, \mathcal{X}_{4, \text { post }}\right)$

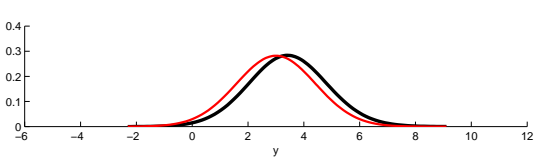

(i-3) $p\left(y \mid \mathcal{X}_{3, \text { pre }}, \mathcal{X}_{3, \text { post }}\right)$

Figure 38S: Bayesian inferences on five datasets, each of which contains $n_{v}=24$ detect data out of $n=48$ samples. 


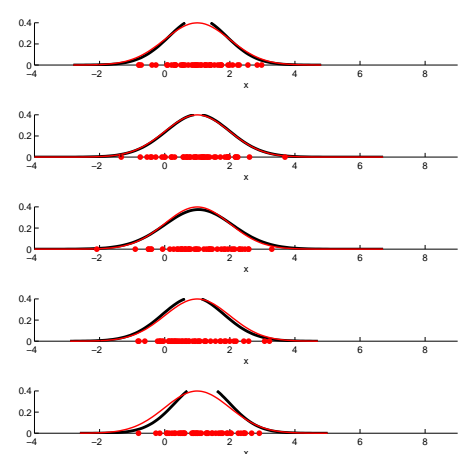

(a) $p\left(c_{\text {post }} \mid \mathcal{X}_{k, \text { post }}\right)$

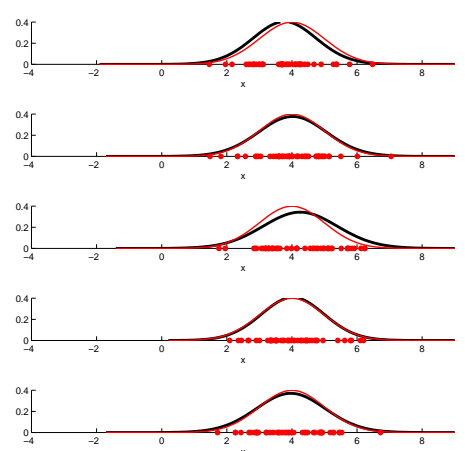

(d) $p\left(c_{\text {pre }} \mid \mathcal{X}_{k, \text { pre }}\right)$

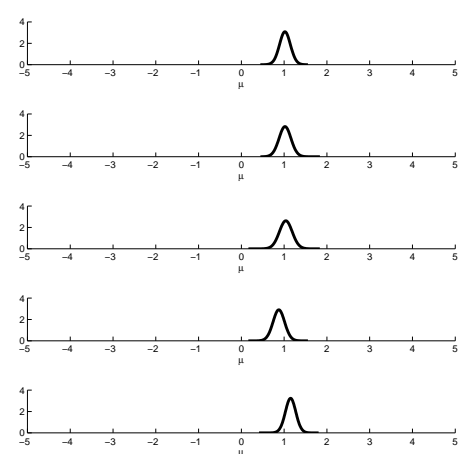

(b) $p\left(\mu_{1} \mid \mathcal{X}_{k, \text { post }}\right)$

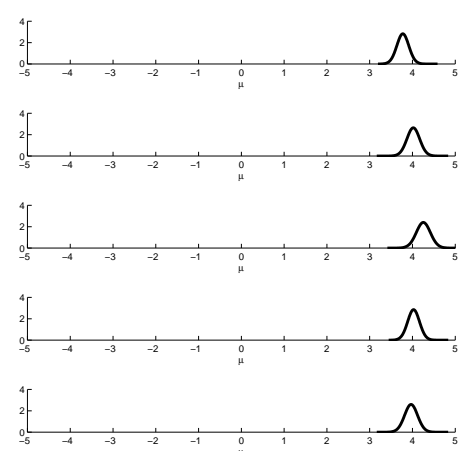

(e) $p\left(\mu_{1} \mid \mathcal{X}_{k, \text { pre }}\right)$

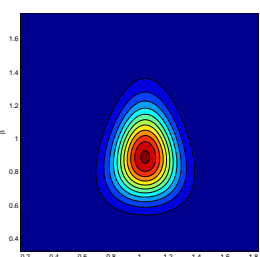

(g-3) $p\left(\mu, \beta \mid \mathcal{X}_{3, \text { post }}\right)$

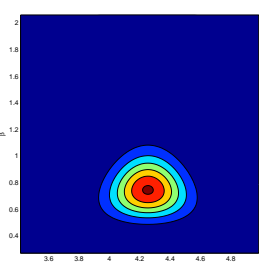

(h-3) $p\left(\mu, \beta \mid \mathcal{X}_{3, \text { pre }}\right)$

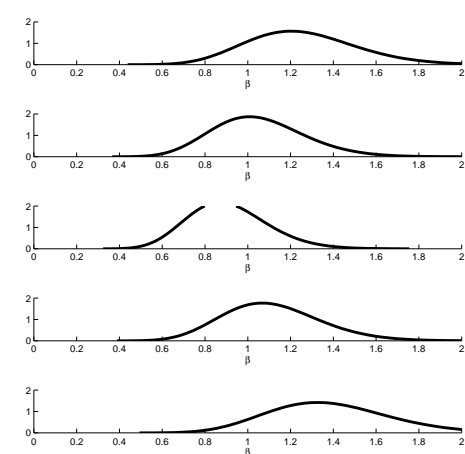

(c) $p\left(\beta_{1} \mid \mathcal{X}_{k, \text { post }}\right)$

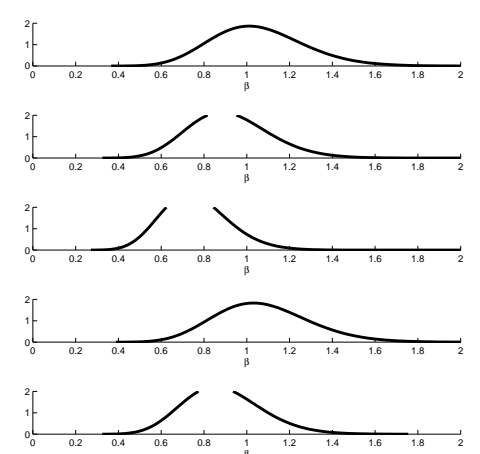

(f) $p\left(\beta_{1} \mid \mathcal{X}_{k, \text { pre }}\right)$

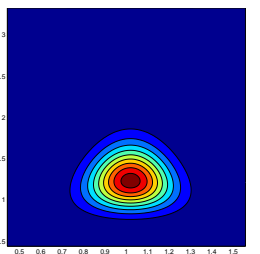

(g-1) $p\left(\mu, \beta \mid \mathcal{X}_{1, \text { post }}\right)$

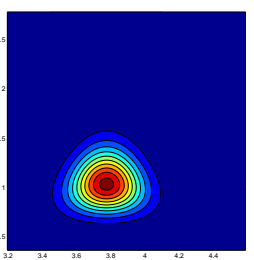

(h-1) $p\left(\mu, \beta \mid \mathcal{X}_{1, \text { pre }}\right)$

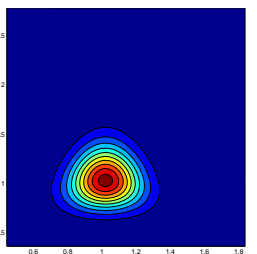

(g-2) $p\left(\mu, \beta \mid \mathcal{X}_{2, \text { post }}\right)$

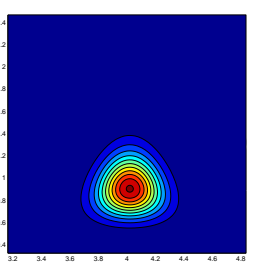

(h-2) $p\left(\mu, \beta \mid \mathcal{X}_{2, \text { pre }}\right)$

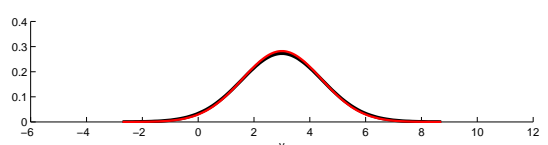

(i-2) $p\left(y \mid \mathcal{X}_{2, \text { pre }}, \mathcal{X}_{2, \text { post }}\right)$

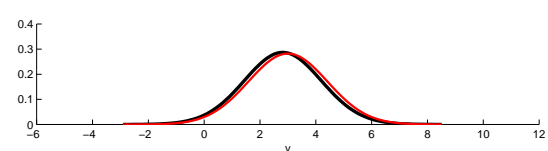

(i-5) $p\left(y \mid \mathcal{X}_{5, \text { pre }}, \mathcal{X}_{5, \text { post }}\right)$

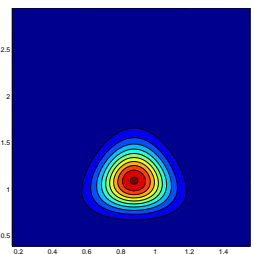

(g-4) $p\left(\mu, \beta \mid \mathcal{X}_{4, \text { post }}\right)$

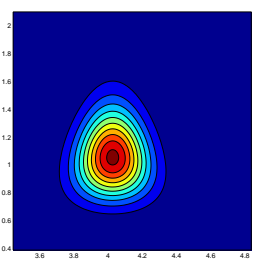

(h-4) $p\left(\mu, \beta \mid \mathcal{X}_{4, \text { pre }}\right)$

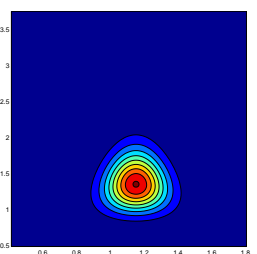

(g-5) $p\left(\mu, \beta \mid \mathcal{X}_{5, \text { post }}\right)$

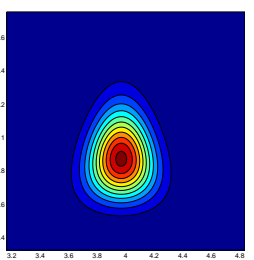

(h-5) $p\left(\mu, \beta \mid \mathcal{X}_{5, \text { pre }}\right)$

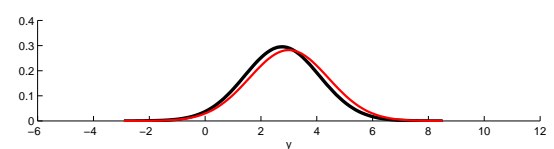

(i-1) $p\left(y \mid \mathcal{X}_{1, \text { pre }}, \mathcal{X}_{1, \text { post }}\right)$

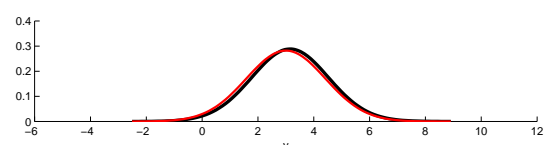

(i-4) $p\left(y \mid \mathcal{X}_{4, \text { pre }}, \mathcal{X}_{4, \text { post }}\right)$

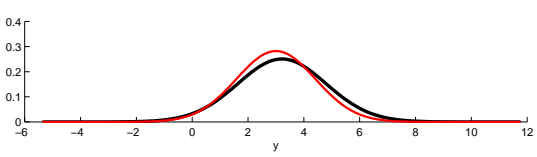

(i-3) $p\left(y \mid \mathcal{X}_{3, \text { pre }}, \mathcal{X}_{3, \text { post }}\right)$

Figure 39S: Bayesian inferences on five datasets, each of which contains $n_{v}=48$ detect data out of $n=48$ samples. 